NBER WORKING PAPER SERIES

\title{
ON THE EQUILIBRIUM PROPERTIES OF NETWORK MODELS WITH HETEROGENEOUS AGENTS
}

\author{
Treb Allen \\ Costas Arkolakis \\ Xiangliang Li \\ Working Paper 27837 \\ http://www.nber.org/papers/w27837 \\ NATIONAL BUREAU OF ECONOMIC RESEARCH \\ 1050 Massachusetts Avenue \\ Cambridge, MA 02138 \\ September 2020, Revised July 2022
}

We thank Truman Bewley, Vasco Carvalho, Xiaohong Chen, Yi Chen, Dave Donaldson, John Geanakoplos, Johannes Horner, Steve Redding, Andres Rodriguez-Clare, Larry Samuelson, Alireza Tahbaz-Salehi, Xinyang Wang, and Ivan Werning, our editor Asher Wolinksy, and four anonymous referees for helpful comments and suggestions. Ari Boyarsky, Joonhyuk Lee, Saptarshi Majumdar, and Fan Wu offered excellent research assistance. This paper has been previously circulated under the title "On the Existence and Uniqueness of Trade Equilibria". The authors acknowledge support by the National Science Foundation under grants SES-1658838 and SES-1658875; Xiangliang Li acknowledges support by the Fundamental Research Funds for the Central Universities under grant 63222067. All errors are our own. The views expressed herein are those of the authors and do not necessarily reflect the views of the National Bureau of Economic Research.

NBER working papers are circulated for discussion and comment purposes. They have not been peer-reviewed or been subject to the review by the NBER Board of Directors that accompanies official NBER publications.

(C) 2020 by Treb Allen, Costas Arkolakis, and Xiangliang Li. All rights reserved. Short sections of text, not to exceed two paragraphs, may be quoted without explicit permission provided that full credit, including $\odot$ notice, is given to the source. 
On the Equilibrium Properties of Network Models with Heterogeneous Agents

Treb Allen, Costas Arkolakis, and Xiangliang Li

NBER Working Paper No. 27837

September 2020, Revised July 2022

JEL No. C6,D85,E23,F4,O18,R13

\section{ABSTRACT}

We consider a broad class of network models where a large number of heterogeneous agents simultaneously interact in many ways. We provide a new theorem that offers an iterative algorithm for calculating an equilibrium and sufficient and "globally necessary" conditions under which the equilibrium is unique. We show that a wide variety of heterogeneous agent economies yield equilibrium representations amenable to our theorem's characterization.

Treb Allen

Department of Economics Dartmouth College

6106 Rockefeller Hall

Hanover, NH 03755

and NBER

treb@dartmouth.edu

Costas Arkolakis

Department of Economics

Yale University, 28 Hillhouse Avenue

P.O. Box 208268

New Haven, CT 06520-8268

and NBER

costas.arkolakis@yale.edu
Xiangliang Li

28 Hillhouse Ave, Department of Economics

Yale University

New Haven, CT 06520-8268

1xlnemo@gmail.com 


\section{Introduction}

The twenty first century has witnessed the rise of big data and big models in the social sciences. Exponential growth in computational capacity combined with access to new microlevel datasets have allowed the empirical implementation of models where large numbers of heterogeneous agents interact simultaneously with each other in myriad ways. Despite the introduction of empirical content to traditionally theoretical fields, unresolved questions about the positive properties of these big models remain. Two concerns - critical for applied work - are particularly pressing: How can we compute the solution of an equilibrium system with hundreds or thousands of heterogeneous agents efficiently? And how do we know that the equilibrium we find is the only possible one?

In this short paper, we answer these questions for a large class of models where many heterogeneous agents simultaneously interact in many ways. In particular, we consider systems where $N$ heterogeneous agents engage in $H$ types of interactions whose equilibrium can be reduced to a set of $N \times H$ equations of the following form:

$$
x_{i h}=\sum_{j=1}^{N} f_{i j h}\left(x_{j 1}, \ldots, x_{j H}\right),
$$

where $\left\{x_{i h}\right\} \in \mathbb{R}_{++}^{N \times H}$ reflect the (strictly positive) equilibrium outcomes for each agent of each interaction and $f_{i j h}: \mathbb{R}_{++}^{H} \rightarrow \mathbb{R}_{++}$are the known (differentiable) functions that govern the interactions between different agents. In particular, $f_{i j h}$ is the function that governs the impact that an interaction with agent $j$ has on agent $i$ 's equilibrium outcome of type $h$. As we illustrate, this formulation is sufficiently general to apply to many types of economic networks - from firm linkages to social networks to the spatial structure of cities.

The main formal result of the paper is a three-part Theorem that offers a unified characterization of the positive properties of equilibrium systems satisfying equation (1). The key insight, loosely speaking, is to simplify the analysis by abstracting from agent heterogeneity and the particular network structure and focusing instead on the strength of economic interactions. Formally, rather than focusing on the $N^{2} \times H$ functions $\left\{f_{i j h}\right\}$ themselves, we instead focus on the $H \times H$ matrix of the uniform bounds of the elasticities $(\mathbf{A})_{h h^{\prime}} \equiv \sup _{i, j}\left(\left|\frac{\partial \ln f_{i j h}}{\partial \ln x_{j h^{\prime}}}\right|\right)$ of the functions. The Theorem characterizes the equilibrium properties of the system based on a single statistic of this matrix: its spectral radius. If its spectral radius is less than one, there exists a unique equilibrium which can be calculated using an iterative algorithm (part i); if its spectral radius is equal to one (with additional restrictions on $\left\{f_{i j h}\right\}$ ), there is at most one equilibrium (part ii); and if its spectral radius is greater than one, then there exist $\left\{f_{i j h}\right\}$ where multiplicity is assured (part iii). 
Part (i) of Theorem 1 relies on a multi-dimensional extension of the contraction mapping theorem to a vector-valued metric known as the Perov Fixed Point Theorem (Perov, 1964; Perov and Kibenko, 1966); we construct such a vector-valued metric by partitioning the space of endogenous variables into subsets, each of which operates in a different metric subspace. This partition is particularly helpful in economic models where heterogeneous agents interact in many ways (i.e. $H$ is large), as it allows us to separate the study of each type of interaction. Part (ii) of Theorem 1 relies on a new contradiction argument, and we show that the knife edge case to which it applies is common in economics, as any constantelasticity system where one of the equilibrium outcomes is a nominal variable (e.g. prices) has a spectral radius no smaller than one. Part (iii) of Theorem 1 is proved by construction; it reveals how the spectral radius being greater than one engenders multiplicity and provides a practical guide for finding multiple solutions. The three parts together demonstrate that the spectral radius of the elasticity matrix is the best condition possible abstracting from the particularities of agent heterogeneity, i.e. it is sufficient and "globally necessary."

To illustrate its broad applicability, we finally apply Theorem 1 to seminal models from many disparate fields in economics. In the main text, we offer two applications; in Online Appendix A, we offer ten more. ${ }^{1}$ First, we extend an urban model in the spirit of Ahlfeldt, Redding, Sturm, and Wolf (2015) to a setting with many types of agents where the payoffs of the choice of residence and workplace depend flexibly on the choices of other agents. Second, we extend a social network model in the spirit of Ballester, Calvó-Armengol, and Zenou (2006) to a setting where agents simultaneously take many actions on potentially many different networks, with the payoffs of each depending flexibly on the actions of other agents. In both cases, we illustrate each part of the Theorem, deriving sufficient conditions guaranteeing convergence to a solution and uniqueness and providing examples of multiplicity if those conditions do not hold. We note that these extensions are contributions in their own right, as they allow heterogeneous agent network models to be brought to bear on important empirical questions. For example, in the urban application incorporating many types of agents enables the "quantitative" study of such issues as spatial segregation and gentrification, whereas in our social network application, our extension enables the analysis of the interdependent nature of different types of actions on different social networks.

\footnotetext{
${ }^{1}$ These include network models with social interactions as in Brock and Durlauf (2001) and Glaeser and Scheinkman (2002), public goods provision in social networks as in Allouch (2015) and Bergstrom, Blume, and Varian (1986), multiple activities as in Chen, Zenou, and Zhou (2018), economic geography models and trade models as in Allen and Arkolakis (2014) and Alvarez and Lucas (2007), trade models with input-output structures as in Caliendo and Parro (2015), input-output production networks as in Acemoglu, Carvalho, Ozdaglar, and Tahbaz-Salehi (2012) and Carvalho, Nirei, Saito, and Tahbaz-Salehi (2021), and demand estimation as in Berry, Levinsohn, and Pakes (1995).
} 


\section{Contribution to the literature}

To highlight the contribution of our paper, it is helpful to compare our approach to four alternatives for characterizing the properties of an equilibrium.

First, one could recursively apply a process of substitution to re-define the equilibrium system as a function of fewer economic interactions. For example, in a simple exchange economy with multiple agents and multiple goods, there are two interactions - buying and selling, which in equilibrium can be summarized by the value of each agent's endowment (wages) and consumption bundle (price index). Alvarez and Lucas (2007) characterize the equilibrium of such a system by first substituting wages into the price index and then analyzing the structure of the model only in terms of wages relying on the gross substitutes property of the system, c.f. Mas-Colell, Whinston, and Green (1995). While feasible for small $H$, the complexity of this approach increases exponentially with the number of interactions in the model, creating a curse of dimensionality for large $H$. Theorem 1 , in contrast, avoids such a curse by simultaneously considering all $H$ interactions and, as we show in Online Appendix A.7, delivers less stringent sufficient conditions for uniqueness even while relaxing the restriction that tariffs are uniform (albeit under an additional assumption that trade costs are "quasi"-symmetric).

Second, one could "stack" all economic outcomes into a single $N H \times 1$ vector and apply standard contraction mapping arguments. The disadvantage of such an approach is that it treats different types of interactions identically - despite the fact that they may play very different roles in the equilibrium system. This results in a loss of information and introduces the possibility that the sufficient conditions may fail despite the system being unique. For example, consider the $N=1 H=2$ system $x_{11}=x_{11}^{\frac{1}{2}} x_{12}^{2}+1, x_{12}=x_{12}^{\frac{1}{2}}+1$. It is straightforward to show that by treating $x_{11}$ and $x_{12}$ as a single vector variable, the standard contraction conditions that the matrix norm (induced by the vector norm) of the system's Jacobian matrix is strictly less than one are not satisfied, whereas the conditions for our Theorem are satisfied.

Third, one could characterize the Jacobian matrix of the equilibrium system directly, e.g. using the results of Hadamard (1906), Gale and Nikaido (1965), or Kehoe (1980). While powerful, such an approach is often impractical in situations with large number of equations, as the Jacobian of equation (1) is of size $N H \times N H$, making it difficult to characterize. In contrast, the conditions provided here depend on a single statistic of an $H \times H$ matrix. And even when the Jacobian can be characterized, the associated conditions required to establish uniqueness may be too stringent, as noted by e.g. Berry, Gandhi, and Haile (2013). For example, consider the system $x_{i}=\sum_{j=1}^{N} K_{i j} x_{j}^{\alpha}$ for $K_{i j}>0$ and $\alpha \in(0,1]$. The $i^{t h}$ diagonal term of its Jacobian is $1-\alpha K_{i i} x_{i}^{\alpha-1}$ which can be negative or positive, violating e.g. the 
classical condition of Gale and Nikaido (1965) that all principal submatrices of the Jacobian have positive determinants. In this case, however, the spectral radius of the elasticity is $\alpha \leq 1$, so uniqueness is established immediately by the Theorem presented here.

Fourth, one could apply the recent results from the social network literature (see e.g. Ballester, Calvó-Armengol, and Zenou (2006), Bramoullé, Kranton, and D'amours (2014), and Parise and Ozdaglar (2019)), which offers conditions for uniqueness based on the structure of the network. One advantage of our approach which instead is based on the elasticities of economic interactions is that the conditions we provide will hold regardless of the network structure, which is useful in settings where the same economic model may be applied to different empirical contexts. For example, in spatial models, the heterogeneity of agents and network structure captures such things like the geography (e.g. trade costs) which are highly context dependent; in contrast, the elasticities govern the strength of economic interactions (e.g. the elasticity of demand) that may be similar across locations. As we show below, these conditions may offer distinct (but complementary) characterizations of the positive properties of existing network games. Another distinction between our approach and those of the social network literature is our focus on the strictly positive domain and range of equation (1); while this is due primarily to our focus on elasticities, we also offer extensions of our results below to cases where the domain and range include zero.

In summary, we approach the $N \times H$ system represented in equation (1) by extracting an $H \times H$ matrix capturing the strength of economic interactions and characterize the equilibrium properties of the system based on a single statistic of that matrix. As a result, Theorem 1 provides a unified understanding of a broad class of heterogeneous agent network models and offers a straightforward and easy-to-check sufficient condition to characterize their equilibrium - reminiscent of how Blackwell (1965) offers straightforward sufficient conditions that have been widely used to characterize the equilibrium of single-agent dynamic models. In doing so - and as the included applications illustrate - it offers both new lessons for existing models and the ability to extend existing frameworks in important directions.

The structure of the remainder of the paper is as follows: Section 2 presents the Theorem and makes seven remarks. Section 3 presents two applications of the result to the fields of urban spatial networks and social networks, respectively. For brevity, the proof of the Theorem is presented in the Appendix. Details of the remarks and ten additional applications are presented in the Online Appendix. 


\section{The Theorem}

Let $\mathcal{N} \equiv\{1, \ldots, N\}$ and $\mathcal{H} \equiv\{1, \ldots, H\}$ correspond to the set of economic agents and the set of economic interactions, respectively. ${ }^{2}$ Let $x$ be an $N$-by- $H$ matrix of endogenous economic outcomes, where for $i \in \mathcal{N}$ and $h \in \mathcal{H}$, we slightly abuse notation and let $x_{i}$ denote $x$ 's $i$ th row and $x_{. h}$ to denote $x$ 's $h$ th column. We restrict our attention to strictly positive $\left\{x_{i h}\right\}_{i \in \mathcal{N}, h \in \mathcal{H}} \in \mathbb{R}_{++}^{N \times H}$ and strictly positive and differentiable $\left\{f_{i j h}\right\}$. Define the elasticity $\epsilon_{i j h, j h^{\prime}}\left(x_{j}\right) \equiv \frac{\partial \ln f_{i j h}\left(x_{j}\right)}{\partial \ln x_{j h^{\prime}}}$, i.e. $\epsilon_{i j h, j h^{\prime}}\left(x_{j}\right)$ is the impact of agent $j^{\prime} s$ outcome of type $h^{\prime}$ on agent $i^{\prime} s$ outcome of type $h$. Finally, define $\rho(\mathbf{A})$ as the spectral radius of matrix $\mathbf{A}$ (i.e. the largest absolute value of its eigenvalues).

Theorem 1. Suppose there exists an $H$-by-H matrix $\mathbf{A}$ such that for all $i, j \in \mathcal{N}, h, h^{\prime} \in \mathcal{H}$, and $x_{j} \in \mathbb{R}_{++}^{H},\left|\epsilon_{i j h, j h^{\prime}}\left(x_{j}\right)\right| \leq(\mathbf{A})_{h h^{\prime}}$. Then:

(i). If $\rho(\mathbf{A})<1$, there exists a unique solution to equation (1) which can be computed by iteratively applying equation (1) with a rate of convergence $\rho(\mathbf{A})$;

(ii). If $\rho(\mathbf{A})=1$ and:

a. For all $i \in \mathcal{N}$ and $h, h^{\prime} \in \mathcal{H}$ when $(\mathbf{A})_{h h^{\prime}} \neq 0$ there exists some $j$ such that for all $x_{j} \in \mathbb{R}_{++}^{H},\left|\epsilon_{i j h, j h^{\prime}}\left(x_{j}\right)\right|<(\mathbf{A})_{h h^{\prime}}$, then equation (1) has at most one solution;

b. For all $x_{j}, \epsilon_{i j h, j h^{\prime}}\left(x_{j}\right)=\alpha_{h h^{\prime}} \in \mathbb{R}$ where $\left|\alpha_{h h^{\prime}}\right|=(\mathbf{A})_{h h^{\prime}}$ for all $i, j \in \mathcal{N}$ and $h, h^{\prime} \in$ $\mathcal{H}$-i.e. $f_{i j h}\left(x_{j}\right)=K_{i j h} \prod_{h^{\prime} \in \mathcal{H}} x_{j h^{\prime}}^{\alpha_{h h^{\prime}}}$ for some $K_{i j h}>0$-then there is at most one columnwise up-to-scale solution, i.e. for any $h \in \mathcal{H}$ and two solutions $x$ and $x^{\prime}$ it must be $x_{. h}^{\prime}=c_{h} x_{. h}$ for some scalar $c_{h}>0$;

(iii). If $\rho(\mathbf{A})>1, N \geq 2 H+1$, and $f_{i j h}\left(x_{j}\right)=K_{i j h} \prod_{h^{\prime} \in \mathcal{H}} x_{j h^{\prime}}^{\alpha_{h h^{\prime}}}$, then there exists some $\left\{K_{i j h}>0\right\}_{i, j \in \mathcal{N}, h \in \mathcal{H}}$ such that equation (1) has multiple solutions that are column-wise up-to-scale different.

Proof. See Appendix A.

Part (i) of Theorem 1 applies the Perov Fixed Point Theorem (Perov, 1964; Perov and Kibenko, 1966) to show that there exists a unique solution and that solution can be computed with an iterative algorithm that converges at a rate $\rho(\mathbf{A})$. In particular, denote equation (1) as $x=T(x)$; then for any initial "guess" of a positive solution $x^{0} \in \mathbb{R}_{++}^{N \times H}$, one simply iterates $x^{1}=T\left(x^{0}\right), x^{2}=T\left(x^{1}\right), x^{3}=T\left(x^{2}\right), \ldots$ until convergence. Intuitively, if (1) represents an agents $i$ 's best response function for action $h$, then this algorithm is simply an iterated best response and part (i) guarantees such best response dynamics will converge to the unique (Nash) equilibrium from any starting point.

\footnotetext{
${ }^{2}$ More generally, the set of agents $\mathcal{N}$ can be countably infinite or uncountably infinite represented by a closed interval.
} 
Part (ii) of Theorem 1 deals with the case of $\rho(\mathbf{A})=1$, which turns out to be a common phenomenon in economic modeling (see Remark 6 below). It establishes uniqueness by imposing extra conditions on the elasticities $\epsilon_{i j h, j h^{\prime}}\left(x_{j}\right)$ : if either the elasticities are strictly smaller than their bounds (part ii.a) or the elasticities are constant (part ii.b) then (up-toscale) uniqueness can be assured.

Finally, since whether or not a system of the form of equation (1) has a unique solution in general depends on the particular function $\left\{f_{i j h}\right\}$, our choice to abstract from this heterogeneity comes at the cost of preventing us from providing necessary conditions for uniqueness. Nonetheless, part (iii) of Theorem 1 shows that the conditions provided are "globally necessary". That is, for any matrix of elasticity bounds A such that $\rho(\mathbf{A})>1$, one can construct a set of functions that govern the interactions $\left\{f_{i j h}\right\}$ with a corresponding $\mathbf{A}$ where multiple equilibria are assured. ${ }^{3}$ Such functions can be constructed even restricting attention only to functions with constant elasticities. Put another way, the sufficient conditions for uniqueness provided in the Theorem 1 are the best that can be provided when abstracting from agent heterogeneity and network structure.

\section{Remarks}

We provide below seven remarks that both facilitate the implementation and extend Theorem 1. Details are presented in Online Appendix B.1. The first four remarks provide extensions to Theorem 1.

Remark 1. (Generalized Domain and Range) Theorem 1 can be extended to both allow the domain of $\left\{f_{i j h}\right\}$ to be a function of the full set of equilibrium outcomes $x$ for all $j$ and allow the range of $\left\{f_{i j h}\right\}$ to be weakly positive, i.e. $f_{i j h}: \mathbb{R}_{++}^{H \times N} \rightarrow \mathbb{R}_{+}$. To do so, we instead require the summation across $j$ to be strictly positive, i.e. $\sum_{j \in \mathcal{N}} f_{i j h}(x)>0$. This alters the conditions in Theorem 1 from a condition on the elasticity $\left|\epsilon_{i j h, j h^{\prime}}\left(x_{j}\right)\right|$ to a condition on the sum of elasticities, i.e. $\sum_{j \in \mathcal{N}}\left|\frac{\partial \ln \sum_{k \in \mathcal{N}} f_{i k h}(x)}{\partial \ln x_{j h^{\prime}}}\right|$ for parts (i) and (ii.a) and $\epsilon_{i j h, j h^{\prime}}\left(x_{j}\right)=\alpha_{h h^{\prime}}$ with $\sum_{j \in \mathcal{N}} \frac{\partial \ln \sum_{k \in \mathcal{N}} f_{i k h}(x)}{\partial \ln x_{j h^{\prime}}}=\alpha_{h h^{\prime}}$ for part (ii.b). The remainder of Theorem 1 and its proof is unchanged. This generalization allows both that the impact that agent $j$ has on agent $i$ through an interaction of type $h$ can depend on the equilibrium outcomes of any other agents (including $i$ 's own outcomes) and for certain agents' interactions to not directly affect the payoffs of others e.g. in a network whose graph is not complete. We apply this remark in Section 3.1.

\footnotetext{
${ }^{3}$ Part (iii) of Theorem 1 extends the result of Allen and Donaldson (2018) to equilibrium systems with more than two equilibrium interactions (i.e. $H>2$ ).
} 
Remark 2. (Weakly Positive Solutions) We can also extend Theorem 1 to consider both a weakly positive domain (and range) of $\left\{f_{i j h}\right\}$ in the following way. Consider the special case of equation (1) where $f_{i j h}: \mathbb{R}_{+}^{H} \rightarrow \mathbb{R}_{+} \equiv K_{i j h} g_{i j h}\left(x_{j 1}, \ldots, x_{j H}\right)$, where $K_{i j h} \geq 0$ and $g_{i j h}\left(x_{j}\right)$ is a function that is continuous, differentiable, and $g_{i j h}\left(x_{j}\right)>0$ for all $x_{j}>0$ so that equation (1) becomes $x_{i h}=\sum_{j=1}^{N} K_{i j h} g_{i j h}\left(x_{j 1}, \ldots, x_{j H}\right)$. This extension allows us to consider the possibility that an equilibrium $x^{*}$ of equation (1) is weakly positive, i.e. for some $i, h, x_{i, h}^{*}=0$. Let non-negative matrix $\mathbf{A}$ represent the bounds of elasticities $\frac{\partial \ln g_{i j h}\left(x_{j}\right)}{\partial \ln x_{j h^{\prime}}}$ for $x_{j}>0$. Then if $\rho(\mathbf{A})<1$ and matrices $\left(K_{i j h}\right)_{i, j \in \mathcal{N}}$ for all $h$ are irreducible, there exists only one strictly positive solution. Weakly positive solutions may exist but will be asymptotically unstable, in the sense of e.g. Weibull (1997). Specifically, for any $\varepsilon>0$ and any $x \in \mathbb{R}_{++}^{N H}$ such that $\left\|x-x^{*}\right\|<\epsilon$ the repeated application of equation (1) from $x$ will diverge away from the weakly positive solution. Intuitively, while there may exist weakly positive equilibria, the best response dynamics from any arbitrarily nearby strictly positive $x$ will diverge away from any of these equilibria (and toward the unique strictly positive solution) when $\rho(\mathbf{A})<1 .{ }^{4}$ We apply this remark in Section 3.2.

Remark 3. (Conditions on Derivatives) While the conditions of Theorem 1 are stated in terms of the size of the elasticities, we can also derive comparable results in terms of derivatives. Note that this approach provides another means of extending Theorem 1 to consider domains and ranges with zero and negative values. Suppose that for all $i \in \mathcal{N}, h \in$ $\mathcal{H}, F_{i h}(x) \equiv \sum_{j} f_{i j h}(x)$ is continuous and has right and left partial derivatives with respect to $x_{j h^{\prime}}$ (denoted as $\frac{\partial_{+} F_{i h}(x)}{\partial x_{j h^{\prime}}}$ and $\left.\frac{\partial_{-} F_{i h}(x)}{\partial x_{j h^{\prime}}}\right)$. Define $\delta_{i h, j h^{\prime}}(x) \equiv \max \left(\left|\frac{\partial_{+} F_{i h}(x)}{\partial x_{j h^{\prime}}}\right|,\left|\frac{\partial_{-} F_{i h}(x)}{\partial x_{j h^{\prime}}}\right|\right)$. Equation (1) has a unique solution if (1) there exists an $H$-by- $H$ matrix A satisfying $\rho(\mathbf{A})<$ 1 such that for all $i \in \mathcal{N}, h, h^{\prime} \in \mathcal{H}, \sum_{j} \delta_{i h, j h^{\prime}}(x) \leq(\mathbf{A})_{h h^{\prime}}$ or $(2)$ there exists an $N$-by- $N$ matrix $\mathbf{B}$ satisfying $\rho(\mathbf{B})<1$ such that for all $i, j \in \mathcal{N}, h \in \mathcal{H}, \sum_{h^{\prime}} \delta_{i h, j h^{\prime}}(x) \leq(\mathbf{B})_{i j}$. Note that the derivatives of equation (1) typically will depend on the particular network structure. Thus this extension of Theorem 1 bears a closer resemblance to existing work on social networks (e.g. Ballester, Calvó-Armengol, and Zenou (2006); Bramoullé, Kranton, and D'amours (2014); Allouch (2015); Parise and Ozdaglar (2019)) but nevertheless offers a complementary characterization. ${ }^{5}$ We apply this result in Online Appendices A.3 and A.4

\footnotetext{
${ }^{4}$ For example, consider $x_{i}=\sum_{j=1}^{2} K_{i j} x_{j}^{\frac{1}{2}}$ for $i \in\{1,2\}$, where $K_{11}=K_{22}=0$ and $K_{21}=K_{12}=1$. This equation has two solutions: $x^{*}=(0,0)^{T}$ and $x^{*}=(1,1)^{T}$. Iterating this equation nearby solution $x^{*}=(0,0)^{T}$ from $x=\left(\epsilon_{1}, \epsilon_{2}\right)^{T}>0$, we get sequence $\left(\epsilon_{2}^{\frac{1}{2}}, \epsilon_{1}^{\frac{1}{2}}\right)^{T},\left(\epsilon_{1}^{\frac{1}{2^{2}}}, \epsilon_{2}^{\frac{1}{2^{2}}}\right)^{T},\left(\epsilon_{2}^{\frac{1}{2^{3}}}, \epsilon_{1}^{\frac{1}{2^{3}}}\right)^{T}$.., which converges to solution $x^{*}=(1,1)^{T}$.

${ }^{5}$ For example, consider $x_{i}=\sum_{j} K_{i j} x_{j}$ for $i \in\{1,2\}$, where $K_{11}=K_{21}=K_{22}=0$ and $K_{12}=4$. Applying the strong monotonicity in Parise and Ozdaglar (2019) (Proposition 3) gives a uniqueness condition that
} 
where we analyze the private provision of multiple public goods and multiple activities on networks.

Remark 4. (Presence of Endogenous Scalars) In addition to equilibrium outcomes for each agent and interaction, certain economic systems also contain endogenous scalars that reflect e.g. the aggregate welfare of the system, as in:

$$
\lambda_{h} x_{i h}=\sum_{j=1}^{N} f_{i j h}\left(x_{j 1}, \ldots, x_{j H}\right),
$$

where $\lambda_{h}>0$ is endogenous. We offer two results for such systems.

The first result concerns the equilibrium system (2) with constant elasticities (as in Theorem 1 part ii.b). For this form, if $\rho(\mathbf{A}) \leq 1$, we have the same conclusion as in part ii.b with the proof unchanged: the $\left\{x_{i h}\right\}$ of any solution is column-wise up-to-scale unique. For $\rho(\mathbf{A})<1$, particularly, it is possible to explicitly subsume the endogenous scalars into the equilibrium outcomes through a change in variables, expressing equation (2) as in equation (1), which in turn implies that the $\left\{x_{i h}\right\}$ are column-wise up-to-scale unique. (Separating the $\left\{x_{i h}\right\}$ and $\left\{\lambda_{h}\right\}$ to determine the scale of $\left\{x_{i h}\right\}$ requires the imposition of further equilibrium conditions, e.g. aggregate labor market clearing conditions).

The second result concerns the equilibrium system (2) for general $f_{i j h}$ with $H$ additional aggregate constraints of the form $\sum_{i=1}^{N} x_{i h}=c_{h}$ for known constants $c_{h}>0$. This system has a unique solution as long as $\rho(\mathbf{A})<\frac{1}{2}$, where $\mathbf{A}$ is defined as in Theorem 1. Intuitively, $\rho(\mathbf{A})<\frac{1}{2}$ ensures that the feedback effect from changes in the endogenous scalar are small enough to continue to ensure a contraction. We apply both these results in Section 3.1.

The next remark facilitates implementation of Theorem 1.

Remark 5. (Change of variables) It is often useful to consider a change of variables of one's original equilibrium system when writing it in the form of equation (1). One important example that can be found in the study of network economics is the following system:

$$
x_{i h}=f_{i h}\left(\left\{\sum_{j \neq i} g_{i j h^{\prime}} x_{j h^{\prime}}\right\}_{h^{\prime} \in \mathcal{H}}\right)
$$

where $\sum_{j \neq i} g_{i j h^{\prime}} x_{j h^{\prime}}$ measures the aggregate behavior of agent $i$ 's peers (see, for example, Glaeser and Scheinkman (2002)). Define $y_{i h} \equiv \sum_{j \neq i} g_{i j h} x_{j h}$ and substitute the expression of $(1-c) I-\frac{K+K^{T}}{2}$ is positive definite for some $c>0$, which requires $\rho\left(\frac{K+K^{T}}{2}\right)<1$. But this condition does not hold because $\rho\left(\frac{K+K^{T}}{2}\right)=2>1$. In contrast, applying the result in this remark gives a uniqueness condition $\rho(K)<1$. 
$x_{j h}$. We then obtain $y_{i h}=\sum_{j \neq i} g_{i j h} f_{j h}\left(\left\{y_{j h^{\prime}}\right\}_{h^{\prime} \in \mathcal{H}}\right)$, which is in the form of (1). We illustrate this technique in Online Appendices A.1 and A.2. Another important example that has found widespread use in trade and spatial economics is the following economic system in which the elasticities are constant (see, for example, Arkolakis, Costinot, and Rodríguez-Clare (2012); Allen and Arkolakis (2014); Redding and Rossi-Hansberg (2017)):

$$
\prod_{h^{\prime} \in \mathcal{H}} x_{i h^{\prime}}^{\gamma_{h h^{\prime}}}=\sum_{j \in \mathcal{N}} K_{i j h} \prod_{h^{\prime} \in \mathcal{H}} x_{i h^{\prime}}^{\rho_{h h^{\prime}}} x_{j h^{\prime}}^{\beta_{h h^{\prime}}}
$$

for all $i \in \mathcal{N}$ and $h^{\prime} \in \mathcal{H}$ where $\gamma_{h h^{\prime}}, \rho_{h h^{\prime}}$, and $\beta_{h h^{\prime}}$ are $\left(h, h^{\prime}\right)$ th cells of matrix $\boldsymbol{\Gamma}, \boldsymbol{R}$, and $\mathbf{B}$, respectively. To transform equation (3) to the form of equation (1), if $\boldsymbol{\Gamma}-\boldsymbol{R}$ is invertible, we can redefine $y_{i h} \equiv \prod_{h^{\prime} \in \mathcal{H}} x_{i h^{\prime}}^{\gamma_{h h^{\prime}}-\rho_{h h^{\prime}}}$. Substituting this definition into the right-hand-side we obtain $y_{i h}=\sum_{j \in \mathcal{N}} K_{i j h} \prod_{h^{\prime} \in \mathcal{H}} y_{j h^{\prime}}^{\alpha_{h h^{\prime}}}$, where $\alpha_{h h^{\prime}}$ is the corresponding element of matrix $\mathbf{B}(\boldsymbol{\Gamma}-\mathbf{R})^{-1}$, which is in the form of equation (1) with $(\mathbf{A})_{h h^{\prime}}=\left|\alpha_{h h^{\prime}}\right|$. Note that a change of variables is not just analytically convenient: the presence of the absolute value operator in Theorem 1 means that a change of variables may reduce the spectral radius, making it more likely that the sufficient conditions for uniqueness are satisfied and improving the speed at which an iterative algorithm converges. ${ }^{6}$ We illustrate this change of variables technique in applications presented in Section 3.1 and Online Appendices A.5, A.6, A.7, and A.10.

The last two remarks offer details about the spectral radius.

Remark 6. (Spectral Radius of 1) In practice, $\rho(\mathbf{A})=1$ is a general phenomenon in economic systems. Indeed, any economic system of the form (3) that is homogeneous of degree 0 in at least one of its arguments will have spectral radius $\rho(\mathbf{A})$ no smaller than 1. Intuitively, such systems require the imposition of additional normalization conditions (e.g. a choice of numeraire) to determine the equilibrium. It is important to note, however, imposing the numeraire prior to characterizing the system will not necessarily reduce its spectral radius. ${ }^{7}$ This highlights the importance of part (ii) of Theorem 1's role for the characterization of the equilibrium properties of such systems. We provide two such economic systems in Online Appendices A.9 and A.10.

\footnotetext{
${ }^{6}$ Consider the simple example $x_{i}=\sum_{j=1}^{N} K_{i j} x_{i}^{\beta} x_{j}^{\alpha}$. Applying Theorem 1 directly (using Remark 1) provides the sufficient uniqueness condition $|\alpha|+|\beta| \leq 1$, but transforming the system using a change of variable $y_{i} \equiv x_{i}^{1-\beta}$ to $y_{i}=\sum_{j=1}^{N} K_{i j} y_{j}^{\frac{\alpha}{1-\beta}}$ provides the sufficient uniqueness condition $\left|\frac{\alpha}{1-\beta}\right| \leq 1$, which is a strictly weaker sufficiency condition (e.g. $\alpha=1, \beta=-\frac{1}{2}$ ).

${ }^{7}$ For example, suppose $N$ locations earn and spend their income on each other's goods with Cobb-Douglas expenditure shares with equilibrium defined as $x_{i}=\sum K_{i j} x_{j}$, where $\sum_{i} K_{i j}=1$. This system has a spectral radius of one. Applying a choice of numeraire (i.e. $x_{1}=1$ ) does not affect the spectral radius, as the bounds of the elasticity of the $N-1$ equilibrium system $x_{i}=K_{i i}+\sum_{j=2}^{N} K_{i j} x_{j}$ remains one.
} 
Remark 7. (Characterization of the Spectral Radius) While it is straightforward to numerically calculate $\rho(\mathbf{A})$ to apply the results of Theorem 1, analytical characterizations are also possible. We offer two results to facilitate such characterization. The first is well known: the Collatz-Wielandt Formula (e.g. see Page 670 in Meyer (2000)), implies that if the summation of each row (or column) of $\mathbf{A}$ is less than 1 , then $\rho(\mathbf{A}) \leq 1$. We illustrate how to use this result to generate intuitive economic conditions for uniqueness in Section 3.1 below. The second is, to our knowledge, new. Define $g(s)$ as the determinant of matrix $s I-\mathbf{A}$ i.e. $g(s)=|s I-\mathbf{A}|$ and denote its $k$-th derivative as $g^{(k)}(s)$. For any constant $s>0, \rho(\mathbf{A}) \leq s$ if and only if $g^{(k)}(s) \geq 0$ for all $k=0,1,2, \ldots, n-1$. We apply this result in Online Appendix A.7.

\section{Applications}

We finally apply Theorem 1 to extend seminal models of urban economics and social networks; in the Online Appendix A, we apply Theorem 1 to ten additional applications. ${ }^{8}$

\subsection{An urban spatial network with many types of agents}

We first consider an urban spatial network based on the work of Ahlfeldt, Redding, Sturm, and Wolf (2015) where agents choose where to live and where to work subject to commuting costs. While we do not explicitly model the land market nor do we incorporate spillovers that decay over space, we extend that framework to incorporate (a) many different types of agents; and (b) flexible productivity and amenity spillovers between agents of different

\footnotetext{
${ }^{8}$ In Online Appendix A.1, we consider a setting where agents make a discrete choice over a large number of possible actions, extending the framework of Brock and Durlauf (2001); in Online Appendix A.2, we consider a setting where agents interact in a large number of non-market ways, extending the framework of Glaeser and Scheinkman (2002); in Online Appendix A.3, we extend a framework where agents trade-off private consumption and public good contributions as in Bergstrom, Blume, and Varian (1986); Allouch (2015); Acemoglu, García-Jimeno, and Robinson (2015) to incorporate multiple types of public goods; in Online Appendix A.4, we consider a network model where agents make multiple actions as in Chen, Zenou, and Zhou (2018) and extend the analysis (from two) to many actions in different networks.; in Online Appendix A.5, we consider an urban setting where spillovers can occur across space, as in Ahlfeldt, Redding, Sturm, and Wolf (2015); in Online Appendix A.6 we show that spatial spillovers-regardless if agglomerative or congestive - can lead to multiplicity in an economic geography framework based on Allen and Arkolakis (2014); in Online Appendix A.7, we provide sufficient conditions for uniqueness in a trade model with intermediate inputs and tariffs as in Alvarez and Lucas (2007); in Online Appendix A.8 we prove the uniqueness of equilibrium in a production network, extending the framework of Acemoglu, Carvalho, Ozdaglar, and Tahbaz-Salehi (2012) to include a CES aggregator across labor and intermediates and between intermediate goods and multiple types of intermediate goods; in Online Appendix A.9, we show that relative sector productivities can be identified to-scale from observed sales in a trade model with input-output linkages as in Caliendo and Parro (2015); and in Online Appendix A.10, we provide conditions under which demand shifters can be identified from market shares, extending the framework of Berry, Levinsohn, and Pakes (1995) to two types of goods.
} 
types, thereby enabling the framework to be applied to a variety of empirically relevant urban interactions, including e.g. segregation, inequality, and gentrification. ${ }^{9}$

Setup A city comprises $i \in\{1, \ldots, N\} \equiv \mathcal{N}$ blocks inhabited by $h \in\{1, \ldots, H\} \equiv \mathcal{H}$ types of agents with measure $\bar{L}_{h}$. Each agent $\nu$ of type $h \in \mathcal{H}$ chooses where to live, denoted by $i \in \mathcal{N}$, and where to work, denoted by $j \in \mathcal{N}$, to maximize her utility:

$$
U_{i j, h}(\nu)=\frac{u_{i h} w_{j h}}{\mu_{i j h}} \varepsilon_{i j h}(\nu)
$$

where $u_{i h}$ and $w_{j h}$ are the value of living and working, respectively, common to all agents of type $h, \mu_{i j h} \geq 1$ is the commuting cost, and $\varepsilon_{i j, h}(\nu)$ is the idiosyncratic preference of agent $\nu$ of type $h$ over location pairs. We assume $\varepsilon_{i j, h}(\nu)$ is extreme value (Frechet) distributed with shape parameter $\theta_{h}>0$. The number of agents of type $h$ who choose to live in location $i$ and work in location $j$ can then be written as:

$$
L_{i j, h}=\frac{\left(\frac{u_{i h} w_{j h}}{\mu_{i j h}}\right)^{\theta_{h}}}{\sum_{(i, j) \in \mathcal{N}^{2}}\left(\frac{u_{i h} w_{j h}}{\mu_{i j h}}\right)^{\theta_{h}}} \bar{L}_{h} .
$$

Spillovers An agent $h$ working in block $j$ produces a numeraire good, for which they are paid their marginal productivity, $A_{j h}$, i.e. $w_{j h}=A_{j h}$. We suppose agents' aggregate location choices also affect $A_{j h}$ and $u_{j h}$. Specifically, $A_{j h}\left(u_{j h}\right)$ depends both on the innate productivity (amenity) of block $j, \bar{A}_{j h}\left(\bar{u}_{i h}\right)$, and the number of each type of agents working (residing) in that location, $\left\{L_{j h^{\prime}}^{W}\right\}_{h^{\prime} \in \mathcal{H}}\left(\left\{L_{j h^{\prime}}^{R}\right\}_{h^{\prime} \in \mathcal{H}}\right)$ i.e.:

$$
A_{j h}=\bar{A}_{j h} f_{h}^{A}\left(\left\{L_{j h^{\prime}}^{W}\right\}_{h^{\prime} \in \mathcal{H}}\right) ; u_{i h}=\bar{u}_{i h} f_{h}^{u}\left(\left\{L_{i h^{\prime}}^{R}\right\}_{h^{\prime} \in \mathcal{H}}\right)
$$

for some functions $f_{h}^{A}: \mathbb{R}_{++}^{H} \rightarrow \mathbb{R}_{++}$and $f_{h}^{u}: \mathbb{R}_{++}^{H} \rightarrow \mathbb{R}_{++}$.

Equilibrium For any geography $\left\{\left\{\mu_{i j h}\right\}_{h \in \mathcal{H}}^{(i, j) \in \mathcal{N}^{2}},\left\{\bar{A}_{i h}, \bar{u}_{i h}\right\}_{h \in \mathcal{H}}^{i \in \mathcal{N}}\right\}$, measure of agents $\left\{\bar{L}_{h}\right\}_{h \in \mathcal{H}}$, spillover functions $\left\{f_{h}^{A}, f_{h}^{u}\right\}_{h \in \mathcal{H}}$, and commuting elasticities $\left\{\theta_{h}\right\}_{h \in \mathcal{H}}$, an equilibrium is a set of workplace and residential populations $\left\{L_{i h}^{W}, L_{i h}^{R}\right\}_{h \in \mathcal{H}}^{i \in \mathcal{N}}$ such that:

$$
L_{i h}^{W}=\sum_{j \in \mathcal{N}} L_{j i, h} ; L_{i h}^{R}=\sum_{j \in \mathcal{N}} L_{i j, h}
$$

\footnotetext{
${ }^{9}$ In Online Appendix A.5, we characterize the equilibrium of an urban model with spatial spillovers.
} 
i.e. the number of workers (residents) of type $h$ in block $i$ is equal to the total number of agents of type $h$ commuting to (from) that location.

We now apply Theorem 1 to characterize the equilibrium of the model; for detailed derivations see Online Appendix B.2.

Theorem 1, part (i): General spillovers For a general set of spillover functions $f_{h}^{A}$ and $f_{h}^{u}$, we suppose that for all $h, h^{\prime}$, and $j$, we have $\left|\frac{\partial \ln f_{h}^{A}}{\partial \ln L_{j h^{\prime}}^{W}}\right| \leq \alpha_{h h^{\prime}}$ and $\left|\frac{\partial \ln f_{h}^{u}}{\partial \ln L_{j h^{\prime}}^{R}}\right| \leq \beta_{h h^{\prime}}$. Then, by substituting equations (5) and (6) into the equilibrium conditions (7), we can derive the following bounds on the elasticities of the workplace and residential populations: $\sum_{j}\left|\frac{\partial \ln L_{i h}^{W}}{\partial \ln L_{j h^{\prime}}^{W}}\right| \leq 2 \theta_{h} \alpha_{h h^{\prime}}, \sum_{j}\left|\frac{\partial \ln L_{i h}^{W}}{\partial \ln L_{j h^{\prime}}^{R}}\right| \leq 2 \theta_{h} \beta_{h h^{\prime}}, \sum_{j}\left|\frac{\partial \ln L_{i h}^{R}}{\partial \ln L_{j h^{\prime}}^{W}}\right| \leq 2 \theta_{h} \alpha_{h h^{\prime}}$, and $\sum_{j}\left|\frac{\partial \ln L_{i h}^{R}}{\partial \ln L_{j h^{\prime}}^{R}}\right| \leq$ $2 \theta_{h} \beta_{h h^{\prime}}$. Therefore by applying Remark 1, part (i) of Theorem 1 yields the following sufficient condition for uniqueness:

$$
\rho\left(\begin{array}{cc}
2 \boldsymbol{\theta} \boldsymbol{\alpha} & 2 \boldsymbol{\theta} \boldsymbol{\beta} \\
2 \boldsymbol{\theta} \boldsymbol{\alpha} & 2 \boldsymbol{\theta} \boldsymbol{\beta}
\end{array}\right)<1
$$

where $\boldsymbol{\alpha}$ and $\boldsymbol{\beta}$ are $H \times H$ matrices with $\left(h, h^{\prime}\right)$ entries $\alpha_{h h^{\prime}}$ and $\beta_{h h^{\prime}}$, respectively and $\boldsymbol{\theta}$ is an $H \times H$ diagonal matrix with $(h, h)$ entry $\theta_{h}$. An equivalent condition for this to hold is that $\rho(\boldsymbol{\theta}(\boldsymbol{\alpha}+\boldsymbol{\beta}))<\frac{1}{2}$. As $\boldsymbol{\theta}$ captures the dispersion force arising from agent's preference heterogeneity (with smaller values indicating greater dispersion) and $\boldsymbol{\alpha}$ and $\boldsymbol{\beta}$ capture the agglomeration/dispersion forces arising from productivities and amenities, respectively (with larger positive values indicating greater agglomeration), these conditions have a simple intuition: uniqueness can be assured when agglomeration forces are small relative to congestion forces (as in Allen and Arkolakis (2014)).

Theorem 1, part (ii): Constant elasticity spillovers Now we consider the special case where the spillover functions $f_{h}^{A}$ and $f_{h}^{u}$ take the following convenient constant elasticity specification: $f_{h}^{A}\left(\left\{L_{j h^{\prime}}^{W}\right\}_{h^{\prime} \in \mathcal{H}}\right)=\prod_{h^{\prime} \in \mathcal{H}}\left(L_{j h^{\prime}}^{W}\right)^{\alpha_{h h^{\prime}}}$ and $f_{h}^{u}\left(\left\{L_{i h^{\prime}}^{R}\right\}_{h^{\prime} \in \mathcal{H}}\right)=\prod_{h^{\prime} \in \mathcal{H}}\left(L_{i h^{\prime}}^{R}\right)^{\beta_{h h^{\prime}}}$. (The $\alpha_{h h^{\prime}}$ and $\beta_{h h^{\prime}}$ here can be negative.) Combining these spillover functions with equation (5), substituting into the equilibrium system (7), and applying the change of variables in Remarks 4 and 5 results in the following system of equilibrium equations:

$$
\tilde{L}_{i h}^{W}=\sum_{j \in \mathcal{N}}\left(\frac{\bar{u}_{j h} \bar{A}_{i h}}{\mu_{j i h}}\right)^{\theta_{h}} \prod_{h^{\prime} \in \mathcal{H}}\left(\tilde{L}_{j h^{\prime}}^{R}\right)^{\gamma_{h, h^{\prime}}^{R}} ; \tilde{L}_{i h}^{R}=\sum_{j}\left(\frac{\bar{u}_{i h} \bar{A}_{j h}}{\mu_{i j h}}\right)^{\theta_{h}} \prod_{h^{\prime} \in \mathcal{H}}\left(\tilde{L}_{j h^{\prime}}^{W}\right)^{\gamma_{h, h^{\prime}}^{W}}
$$

where $\left[\gamma_{h, h^{\prime}}^{R}\right] \equiv \boldsymbol{\theta} \boldsymbol{\beta}(\mathbf{I}-\boldsymbol{\theta} \boldsymbol{\beta})^{-1}$, and $\left[\gamma_{h, h^{\prime}}^{W}\right] \equiv \boldsymbol{\theta} \boldsymbol{\alpha}(\mathbf{I}-\boldsymbol{\theta} \boldsymbol{\alpha})^{-1}$. This $2 \times N \times H$ system of equations is a special case of the equation (1) and as a result, the uniqueness of the 
equilibrium depends on the spectral radius of the following $2 H \times 2 H$ matrix:

$$
\mathbf{A} \equiv\left(\begin{array}{cc}
0 & \left|\boldsymbol{\theta} \boldsymbol{\beta}(\mathbf{I}-\boldsymbol{\theta} \boldsymbol{\beta})^{-1}\right| \\
\left|\boldsymbol{\theta} \boldsymbol{\alpha}(\mathbf{I}-\boldsymbol{\theta} \boldsymbol{\alpha})^{-1}\right| & 0
\end{array}\right)
$$

From part ii.b of Theorem 1, there is at most one equilibrium as long as the spectral radius of $\mathbf{A}$ is not greater than one, i.e. $\rho(\mathbf{A}) \leq 1$. It can be shown that this condition is strictly weaker than the condition required in the case of general spillovers given above: this arises because the assumed functional form of spillovers allows us to accommodate directly the feedback loop generated by the endogenous welfare through the change of variables.

Theorem 1, part (iii): Multiplicity We consider a simple economy with two identical city blocks with symmetric commuting $\operatorname{costs}\left(N=2, \bar{A}_{i h}=\bar{u}_{i h}=1, \mu_{i j}=\mu\right.$ if $i \neq j$, and $\mu=1$ if $i=j)$, a single type of agent $(H=1)$, a unitary commuting elasticity $(\theta=1)$, and the Cobb-Douglas spillover function from the previous section with equal productivity and amenity spillovers $(\alpha=\beta)$. Part iii of Theorem 1 implies that for any $\alpha=\beta>\frac{1}{2}$, there will exist a $\mu>1$ such that there are multiple equilibria. Online Appendix Figure 1 plots the two equilibrium conditions as a function of the relative number of agents employed and living in the first location. As long as the commuting cost $\mu$ is sufficiently large, for any $\alpha=\beta>\frac{1}{2}$ there are three possible equilibria: one in which there are an equal number of workers and residents in each location and another two where one of two the locations has a greater number of workers and residents to take advantage of the agglomeration economies.

Comparison to previously known results In the $H=1$ constant elasticity case, Ahlfeldt, Redding, Sturm, and Wolf (2015) prove the existence and uniqueness of an equilibrium in the absence of productivity and amenity spillovers where the only forces present are congestion forces due to the inelastic supply of land and the idiosyncratic preferences of agents, i.e. $\alpha<0$ and $\beta<0$ in our notation. Here we show for any finite $H \geq 1$ and any $f_{i j h}$ that uniqueness is assured even if some forces are agglomerative, so long as congestion forces are greater in strength-formally $\rho(\boldsymbol{\theta}(\boldsymbol{\alpha}+\boldsymbol{\beta}))<\frac{1}{2}$ in the general case.

\subsection{Social interactions with many types of networks}

We now consider a social network based on the work of Ballester, Calvó-Armengol, and Zenou (2006) (as summarized in the review article of Jackson and Zenou (2015)) where agents' payoffs depend both on their own actions as well as the actions of others in their social network. We extend that framework to incorporate (a) flexible impacts of others' actions 
on one's own payoffs; (b) many different types of actions; and (c) many different types of networks. Allowing agents' different types of actions through different networks - and for those choices to flexibly affect the payoffs of all other agents' actions - enables the study of a variety of empirically relevant social interactions, including e.g. the interdependent nature of different types actions on different social networks (friends, family, work, etc).

Setup There are $i \in\{1, \ldots, N\} \equiv \mathcal{N}$ agents, each of whom decides how much effort $x_{i h}$ to exert on each activity $h \in\{1, \ldots, H\} \equiv \mathcal{H}$. Agent $i$ 's payoff from activity $h$ is:

$$
u_{i h}\left(\left\{x_{j h^{\prime}}\right\}_{j \in \mathcal{N}}^{h^{\prime} \in \mathcal{H}}\right)=c_{i h} x_{i h}-\frac{x_{i h}^{2}}{2}+x_{i h} \sum_{j \neq i} f_{i j h}\left(\left\{x_{j h^{\prime}}\right\}_{h^{\prime} \in \mathcal{H}}\right)
$$

where $c_{i h}>0$ is the (constant) marginal own benefit of effort, costs are quadratic in effort, and $f_{i j h}(\cdot) \geq 0$ is a function capturing the network of type $h$ and how others' efforts in all activities affect agent $i$ 's payoff in activity $h$. Agent $i$ 's overall utility is given by:

$$
u_{i}(x)=m\left[u_{i 1}(x), \ldots, u_{i H}(x)\right]
$$

where $m(\cdot)$ is a monotonic function increasing in each of its arguments.

Example For the purpose of illustration, consider a simple example with $H=2$ where $f_{i j 1}\left(\left\{x_{j h^{\prime}}\right\}_{h^{\prime} \in \mathcal{H}}\right)=K_{i j 1} x_{j 1}^{\alpha_{11}} x_{j 2}^{\alpha_{12}}$ and $f_{i j 2}\left(\left\{x_{j h^{\prime}}\right\}_{h^{\prime} \in \mathcal{H}}\right)=K_{i j 2} x_{j 1}^{\alpha_{21}} x_{j 2}^{\alpha_{22}}$. Here, $\left\{x_{j 1}\right\}_{j \in \mathcal{N}}$ and $\left\{x_{j 2}\right\}_{j \in \mathcal{N}}$ can be agents' incomes and educations, respectively; correspondingly, $\left\{K_{i j 1}\right\}_{i, j \in \mathcal{N}}$ and $\left\{K_{i j 2}\right\}_{i, j \in \mathcal{N}}$ reflect the economic and education networks, through which incomes and educations are determined, with $\left\{\alpha_{i j}\right\}_{i, j \in\{1,2\}}$ capturing the interaction between incomes and educations.

Equilibrium Agent $i$ choose her efforts $\left\{x_{i h}\right\}_{h \in \mathcal{H}}$ to maximize her utility $u_{i}(x)$. The first order conditions give the best response function of agent $i \in \mathcal{N}$ for action $h \in \mathcal{H}$ to all other agents actions:

$$
x_{i h}=c_{i h}+\sum_{j \neq i} f_{i j h}\left(\left\{x_{j h^{\prime}}\right\}_{h^{\prime} \in \mathcal{H}}\right)
$$

which is a special case of equation (1) (where $\left.f_{i i h}\left(x_{j 1}, \ldots, x_{j H}\right)=c_{i h}\right)$. We note that Ballester, Calvó-Armengol, and Zenou (2006) consider a single network $(H=1)$ and a linear spillover function $\left(f_{i j}\left(x_{j}\right)=g_{i j} x_{j}\right)$.

Theorem 1, part (i): General spillovers Suppose that the elasticities of the spillover function can be bounded, i.e. for all $h, h^{\prime} \in \mathcal{H}$ there exists an $\alpha_{h h^{\prime}} \geq 0$ such that $\left|\frac{\partial \ln f_{i j h}}{\partial \ln x_{j h^{\prime}}}\right| \leq \alpha_{h h^{\prime}}$ 
for all $\left\{x_{j h^{\prime}}\right\}_{h^{\prime} \in \mathcal{H}}$. Let $\mathbf{A}$ be the $H \times H$ matrix whose $\left(h, h^{\prime}\right)$ element is $\alpha_{h h^{\prime}}$. From Theorem 1 part (i), there exists a unique strictly positive equilibrium if $\rho(\mathbf{A})<1$. Moreover, that equilibrium can be reached from any initial strictly positive starting point $\left\{x_{j h^{\prime}}^{0}\right\}_{j \in \mathcal{N}}^{h^{\prime} \in \mathcal{H}}$ by iteration of equation (9). Note that the iterative procedure here has the simple economic intuition as an application of best-response dynamics, i.e. from any initial starting point, the unique equilibrium can be reached as an iterative application of agents' best-responses (see e.g. section 6 of Parise and Ozdaglar (2019)). Finally, while there may also be weakly positive solutions, from Remark 2 any such solutions will be asymptotically unstable, in the sense of e.g. Weibull (1997). ${ }^{10}$

Theorem 1, part (ii): Constant elasticity spillovers Consider the above example of income and education networks. Then from Theorem 1 part (ii), there is at most one equilibrium if $\rho(\mathbf{A}) \leq 1$

Theorem 1, part (iii): Multiplicity It is sufficient to consider a two agent single network $(N=2, H=1)$ with constant elasticity social spillover $f_{i j}=g_{i j} x_{j}^{\alpha}$. For any $\alpha>1$ it is straightforward to confirm that the payoff structure of $c_{1}=c_{2}=1-\frac{1}{2 \alpha}$ and $g_{12}=g_{21}=\frac{1}{2 \alpha}$ has at least two solutions: a low-effort symmetric equilibrium $\left(x_{1}, x_{2}\right)=(1,1)$ and a higheffort symmetric equilibrium $\left(x_{1}, x_{2}\right)=(M, M)$, where $M$ is the maximal root of the equation $x^{\alpha}-2 \alpha x+2 \alpha-1=0$.

Comparison to previously known results We view our results are complementary to existing results in the social network literature.

In the baseline network model where $H=1$ and $f_{i j}=g_{i j} x_{j}$, Ballester, Calvó-Armengol, and Zenou (2006) show that there exists a unique interior solution if $\rho(\mathbf{G})<1$, where $\mathbf{G}$ is the $N \times N$ matrix with $(i, j)$ element $g_{i j}$, i.e. they provide a condition on the spectral radius of the network structure. In contrast, Theorem 1.ii.a shows there exists at most one interior equilibrium, as the elasticities of $f_{i j}=g_{i j} x_{j}$ and $f_{i i}=c_{i}$ are equal to and smaller than 1 , respectively. Combining these results in the $\alpha=1$ case then illustrates that the condition $\rho(\mathbf{G})<1$ guarantees the existence of an interior equilibrium (indeed, if $\rho(\mathbf{G}) \geq 1$, there exists no interior solution).

Bramoullé, Kranton, and D'amours (2014) and Galeotti, Golub, and Goyal (2020) extend the Ballester, Calvó-Armengol, and Zenou (2006) framework to the case where actions can be substitutes by allowing possibly negative $\mathbf{G}$ and offer similar conditions for uniqueness as

\footnotetext{
${ }^{10}$ In Online Appendix A.4, we extend the analysis here to consider the uniqueness of weakly positive solutions in the setting where the best response functions are linear (i.e. $\rho(\mathbf{A})=1$ ).
} 
Ballester, Calvó-Armengol, and Zenou (2006) based on the network structure. While Theorem 1 does not allow negative $f_{i j h}$, it can incorporate situations where actions are substitutes through negative payoff elasticities. ${ }^{11}$ In the example above, income and education can be substitutes if $\alpha_{12}$ and $\alpha_{21}$ are negative. Moreover, while there may exist non-interior weakly positive equilibria, Remark 2 guarantees that the only stable equilibria is the unique strictly positive solution when the spectral radius $\rho(\mathbf{A})<1$.

As in Allouch (2015); Acemoglu, García-Jimeno, and Robinson (2015) and Chen, Zenou, and Zhou (2018), the setup above also extends the Ballester, Calvó-Armengol, and Zenou (2006) framework to include nonlinearity and multiple activities. However, the setup above also extends the framework to allow for multiple networks, something (to the best of our knowledge) for which positive properties have not been previously characterized, despite the empirical importance of simultaneous social interactions across multiple types networks (see e.g. Christakis and Fowler (2009); Banerjee, Chandrasekhar, Duflo, and Jackson (2013)). Our characterization emphasizes that the positive properties of the equilibrium multi-network system can be characterized in terms of a single statistic: the spectral radius of the matrix of the elasticities of the social interactions.

\section{Conclusion}

In this short paper, we provide sufficient conditions for the uniqueness and computation of the equilibrium for a broad class of models with large numbers of heterogeneous agents simultaneously interacting in a large number of ways. The conditions are written in terms of the elasticities of the economic interactions across agents. We illustrate that a wide variety of heterogeneous agent economies yield equilibrium representations amenable to our theorem's characterization, thereby contributing to our understanding of the big models needed to interpret big data.

By construction, the conditions provided here depend only on the uniform bound of the elasticities of agent's interactions on each other's outcomes rather than the particular form of the network; that is, the conditions provided abstract from agent heterogeneity and network structure. We show that should the conditions provided not hold, there exist network models for which multiplicity is guaranteed, i.e. our conditions are "globally" necessary. However, an outstanding and important question remains about how agent heterogeneity and network structure itself shapes the positive properties of model equilibria.

\footnotetext{
${ }^{11}$ Note that Theorem 1's parallel result, Remark 3, does allow negative $f_{i j h}$. Remark 3 is also complementary with existing works on social networks by enabling the characterization of nonsymmetric networks (see Footnote 5) and settings with multiple actions in multiple networks (see Online Appendices A.3 and A.4).
} 


\section{References}

Acemoglu, D., V. M. Carvalho, A. Ozdaglar, and A. Tahbaz-Salehi (2012): "The network origins of aggregate fluctuations," Econometrica, 80(5), 1977-2016.

Acemoglu, D., C. García-Jimeno, and J. A. Robinson (2015): "State capacity and economic development: A network approach," American Economic Review, 105(8), 2364-2409.

Ahlfeldt, G. M., S. J. Redding, D. M. Sturm, And N. Wolf (2015): "The economics of density: Evidence from the Berlin Wall," Econometrica, 83(6), 2127-2189.

Allen, T., And C. Arkolakis (2014): "Trade and the Topography of the Spatial Economy," The Quarterly Journal of Economics.

Allen, T., C. Arkolakis, And Y. Takahashi (2020): "Universal gravity," Journal of Political Economy, 128(2), 393-433.

Allen, T., And D. Donaldson (2018): "The geography of path dependence," Unpublished manuscript.

Allouch, N. (2015): "On the private provision of public goods on networks," Journal of Economic Theory, $157,527-552$.

Alvarez, F., AND R. E. Lucas (2007): "General Equilibrium Analysis of the Eaton-Kortum Model of International Trade," Journal of Monetary Economics, 54(6), 1726-1768.

Arkolakis, C., A. Costinot, And A. Rodríguez-Clare (2012): "New Trade Models, Same Old Gains?," American Economic Review, 102(1), 94-130.

Ballester, C., A. Calvó-Armengol, And Y. Zenou (2006): "Who's who in networks. Wanted: The key player," Econometrica, 74(5), 1403-1417.

Banerjee, A., A. G. Chandrasekhar, E. Duflo, and M. O. Jackson (2013): "The diffusion of microfinance," Science, 341(6144), 1236498.

Bergstrom, T., L. Blume, And H. Varian (1986): "On the private provision of public goods," Journal of Public Economics, 29(1), 25-49.

Berry, S., A. Gandhi, And P. Haile (2013): "Connected substitutes and invertibility of demand," Econometrica, 81(5), 2087-2111.

Berry, S., J. Levinsohn, And A. Pakes (1995): "Automobile Prices in Market Equilibrium," Econometrica, 63(4), 841-890.

Blackwell, D. (1965): "Discounted dynamic programming," The Annals of Mathematical Statistics, 36(1), $226-235$.

Bramoullé, Y., R. Kranton, And M. D'amours (2014): "Strategic interaction and networks," American Economic Review, 104(3), 898-930.

Brock, W. A., And S. N. Durlauf (2001): "Discrete choice with social interactions," The Review of Economic Studies, 68(2), 235-260.

Caliendo, L., And F. Parro (2015): "Estimates of the Trade and Welfare Effects of NAFTA," The Review of Economic Studies, 82(1), 1-44.

Carvalho, V. M., M. Nirei, Y. U. Saito, And A. Tahbaz-Salehi (2021): "Supply chain disruptions: Evidence from the great east Japan earthquake," The Quarterly Journal of Economics, 136(2), 1255-1321. 
Carvalho, V. M., And A. Tahbaz-Salehi (2019): "Production networks: A primer," Annual Review of Economics, 11, 635-663.

Chen, Y.-J., Y. Zenou, And J. Zhou (2018): "Multiple activities in networks," American Economic Journal: Microeconomics, 10(3), 34-85.

Christakis, N. A., AND J. H. Fowler (2009): Connected: The surprising power of our social networks and how they shape our lives. Little, Brown Spark.

Eaton, J., AND S. Kortum (2002): "Technology, Geography and Trade," Econometrica, 70(5), 1741-1779.

Fine, B., And G. Rosenberger (1997): The Fundamental Theorem of Algebra. Springer Science \& Business Media.

Gale, D., AND H. NikAido (1965): "The Jacobian matrix and global univalence of mappings," Mathematische Annalen, 159(2), 81-93.

Galeotti, A., B. Golub, And S. Goyal (2020): "Targeting interventions in networks," Econometrica, 88(6), 2445-2471.

Glaeser, E., AND J. Scheinkman (2002): "Non-Market Interactions," in In Advances in Economics and Econometetrics: Theory and Applications, Eighth World Congress, ed. by M. Armstrong, and R. Porter. Cambridge, UK: Cambridge University Press.

Hadamard, J. (1906): "Sur les transformations ponctuelles," Bull. Soc. Math. France, 34, 71-84.

JaCKSOn, M. O., AND Y. Zenou (2015): "Games on networks," in Handbook of game theory with economic applications, vol. 4, pp. 95-163. Elsevier.

Kenoe, T. J. (1980): "An index theorem for general equilibrium models with production," Econometrica: Journal of the Econometric Society, pp. 1211-1232.

Mas-Colell, A., M. D. Whinston, And J. R. Green (1995): Microeconomic Theory. Oxford University Press, Oxford, UK.

Meyer, C. D. (2000): Matrix analysis and applied linear algebra. Siam.

Parise, F., AND A. Ozdaglar (2019): "A variational inequality framework for network games: Existence, uniqueness, convergence and sensitivity analysis," Games and Economic Behavior, 114, 47-82.

Perov, A. I. (1964): "On the Cauchy problem for a system of ordinary differential equations (in Russian)," Approximate Methods of Solving Differential Equations. Kiev. Naukova Dumka.

Perov, A. I., AND A. Kibenko (1966): "On a certain general method for investigation of boundary value problems (in Russian)," Izvestiya Rossiiskoi Akademii Nauk. Seriya Matematicheskaya, 30(2), 249-264.

Redding, S. J., And E. A. Rossi-Hansberg (2017): "Quantitative Spatial Economics," Annual Review of Economics, 9(1).

Weibull, J. (1997): Evolutionary Game Theory, vol. 1. The MIT Press, 1 edn. 


\section{A Proof of Theorem 1}

We start by reminding readers of the Perov fixed point theorem (Perov, 1964; Perov and Kibenko, 1966), which is a multi-dimensional extension of the standard contraction mapping theorem and used in the proof of Theorem 1 part (i):

Theorem A.1. (Perov Fixed Point Theorem) Let $\left\{\left(X_{h}, d_{h}\right)\right\}_{h=1,2, \ldots, H}$ be $H$ metric spaces where $X_{h}$ is a set and $d_{h}$ is its corresponding metric. Define $X \equiv X_{1} \times X_{2} \times \ldots \times X_{H}$, and $d$ : $X \times X \rightarrow \mathbb{R}_{+}^{H}$ such that for $x=\left(x_{1, \ldots}, x_{H}\right), x^{\prime}=\left(x_{1, \ldots}^{\prime}, x_{H}^{\prime}\right) \in X, d\left(x, x^{\prime}\right)=\left(\begin{array}{c}d_{1}\left(x_{1}, x_{1}^{\prime}\right) \\ \ldots \\ d_{H}\left(x_{H}, x_{H}^{\prime}\right)\end{array}\right)$.

Given operator $T: X \rightarrow X$, suppose for any $x, x^{\prime} \in X$

$$
d\left(T(x), T\left(x^{\prime}\right)\right) \leq \mathbf{A} d\left(x, x^{\prime}\right),
$$

where $\mathbf{A}$ is a non-negative matrix and the inequality is entry-wise. Denote $\rho(\mathbf{A})$ as the spectral radius of $\mathbf{A}$. If $\rho(\mathbf{A})<1$ and for all $h=1,2, \ldots, H,\left(X_{h}, d_{h}\right)$ is complete, there exists a unique fixed point of $T$, and for any $x \in X$, the sequence of $x, T(x), T(T(x)), \ldots$ converges to the fixed point of $T$.

We now proceed to the analysis of equation (1). Notice that equation (1) can be written as $\ln x_{i h}=\ln \sum_{j \in \mathcal{N}} f_{i j h}\left(\exp \ln x_{j}\right)$. Denote the leftside of this equation $\ln x_{i h}$ as $y_{i h}$ for all $h \in \mathcal{H} i \in \mathcal{N}$ and furthermore denote its right side $\ln \sum_{j \in \mathcal{N}} f_{i j h}\left(\exp y_{j}\right)$ as function $g_{i h}(y)$, we thus have:

$$
\frac{\partial g_{i h}}{\partial y_{j h^{\prime}}}=\frac{\epsilon_{i j h, j h^{\prime}}\left(\exp y_{j}\right) f_{i j h}\left(\exp y_{j}\right)}{\sum_{j \in \mathcal{N}} f_{i j h}\left(\exp y_{j}\right)}
$$

Given any $y$ and $y^{\prime}$, according to the mean value theorem, for each $i$ and $h$, there exists $\hat{y}=\left(1-t_{i h}\right) y+t_{i h} y^{\prime}$ where $t_{i h} \in[0,1]$ such that:

$$
\begin{aligned}
g_{i h}(y)-g_{i h}\left(y^{\prime}\right) & =\nabla g_{i h}(\hat{y})\left(y-y^{\prime}\right) \\
& =\sum_{j \in \mathcal{N}, h^{\prime} \in \mathcal{H}} \frac{\partial g_{i h}(\hat{y})}{\partial y_{j h^{\prime}}}\left(y_{j h^{\prime}}-y_{j h^{\prime}}^{\prime}\right) .
\end{aligned}
$$

Part i: Combine the above two equations (11) and (12) with condition $\left|\epsilon_{i j h, j h^{\prime}}\left(x_{j}\right)\right| \leq$ $(\mathbf{A})_{h h^{\prime}}$, we have

$$
\begin{aligned}
\left|g_{i h}(y)-g_{i h}\left(y^{\prime}\right)\right| & \leq \sum_{j \in \mathcal{N}, h^{\prime} \in \mathcal{H}} \frac{(\mathbf{A})_{h h^{\prime}} f_{i j h}\left(\exp \hat{y}_{j}\right)}{\sum_{j \in \mathcal{N}} f_{i j h}\left(\exp \hat{y}_{j}\right)}\left|y_{j h^{\prime}}-y_{j h^{\prime}}^{\prime}\right| \\
& \leq \sum_{h^{\prime} \in \mathcal{H}}(\mathbf{A})_{h h^{\prime}} \max _{j \in \mathcal{N}}\left|y_{j h^{\prime}}-y_{j h^{\prime}}^{\prime}\right|
\end{aligned}
$$

For any $h \in H$, define metric $d_{h}\left(y_{h}, y_{h}^{\prime}\right)=\max _{j \in \mathcal{N}}\left|y_{j h}-y_{j h}^{\prime}\right|$ on space $Y_{h} \equiv \mathbb{R}^{N}$. Furthermore, define $Y=Y_{1} \times Y_{2} \times \ldots \times Y_{H}$ and $d\left(y, y^{\prime}\right)=\left[d_{h}\left(y_{h}, y_{h}^{\prime}\right)\right]$ for $y, y^{\prime} \in Y$. Notice that inequality (13) then becomes $d\left(g(y), g\left(y^{\prime}\right)\right) \leqq \mathbf{A} d\left(y, y^{\prime}\right)$. Thus we can apply the Perov Fixed Point Theorem to obtain the desired results (existence, uniqueness and computation). 
For the purpose of the computation, instead of applying the iterative procedure in the space $Y=\mathbb{R}^{N \times H}$ according to Theorem A.1, it is equivalent to do so in the space where $x$ lies on, i.e. $\mathbb{R}_{++}^{N \times H}$.

Part ii.a: We proceed by contradiction. Suppose there are two distinct solutions $y$ and $y^{\prime}$ i.e. $y_{i h}=g_{i h}(y)$ and $y_{i h}^{\prime}=g_{i h}\left(y^{\prime}\right)$. Substitute these two solutions into equation (12) and we then have $y_{i h}-y_{i h}^{\prime}=\sum_{j \in \mathcal{N}, h^{\prime} \in \mathcal{H}} \frac{\partial g_{i h}(\hat{y})}{\partial y_{j h^{\prime}}}\left(y_{j h^{\prime}}-y_{j h^{\prime}}^{\prime}\right)$. In this equation, if $(\mathbf{A})_{h h^{\prime}}>0$, according to the condition in Part (ii.a) and equation (11), for some $j\left|\frac{\partial g_{i h}}{\partial y_{j h^{\prime}}}\right|<\frac{(\mathbf{A})_{h h^{\prime}} f_{i j h}\left(\exp y_{j}\right)}{\sum_{j \in \mathcal{N}} f_{i j h}\left(\exp y_{j}\right)}$, so that:

$$
\left|y_{i h}-y_{i h}^{\prime}\right|<\sum_{h^{\prime} \in \mathcal{H}}(\mathbf{A})_{h h^{\prime}} \max _{j \in \mathcal{N}}\left|y_{j h^{\prime}}-y_{j h^{\prime}}^{\prime}\right|
$$

Thus we have vector inequality $d\left(y, y^{\prime}\right) \leq \mathbf{A} d\left(y, y^{\prime}\right)$. Within this vector inequality, each sub-inequality strictly holds as long as its right side is not zero. Since $y$ and $y^{\prime}$ are distinct, $d\left(y, y^{\prime}\right)$ is a nonzero vector. Thus according to the Collatz-Wielandt Formula (i.e. $\rho(\mathbf{A})=$ $\left.\max _{d \in \mathbb{R}_{+}^{H}, d \neq 0} \min _{\substack{1 \leq h \leq H \\ d_{h} \neq 0}} \frac{(\mathbf{A} d)_{h}}{d_{h}}\right)$, we have $\rho(\mathbf{A})>1$, which is a contradiction.

Part ii.b: We again proceed by contradiction. Suppose a pair of solutions $x$ and $x^{\prime}$ to equation (1) exists that are column-wise up-to-scale different. Then for some $h, y_{. h} \equiv$ $\ln x_{. h}$ and $y_{. h}^{\prime} \equiv \ln x_{. h}^{\prime}$ are different up to addition by a constant, i.e. for some $h d_{h} \equiv$ $\operatorname{minmax}_{s \in \mathbb{R}}\left|y_{j h}-y_{j h}^{\prime}+s\right|>0$. Let $d=\left[d_{h}\right]$ be the resulting nonzero vector. For any $h \in \mathcal{H}$, assume the pair of $s_{h}$ and $j_{h}$ reach the min-max in the definition of $d_{h}$, that is, $d_{h}=$ $\left|y_{j_{h} h}-y_{j_{h} h}^{\prime}+s_{h}\right|$. The definition of $d_{h}$ implies the following three properties: (1) For any $h^{\prime}$ and $j,\left|y_{j h^{\prime}}-y_{j h^{\prime}}^{\prime}+s_{h^{\prime}}\right| \leq d_{h^{\prime}} ;(2)$ For any $h^{\prime}$, if $d_{h^{\prime}}>0$, there must exist some $j \in \mathcal{N}$ such that $\left|y_{j h^{\prime}}-y_{j h^{\prime}}^{\prime}+s_{h^{\prime}}\right|<d_{h^{\prime}}$; and (3) For any $h$ and an arbitrary constant $\hat{s}_{h}, d_{h} \leq$ $\max _{i \in \mathcal{N}}\left|y_{i h}-y_{i h}^{\prime}+\hat{s}_{h}\right|$. Substitute $\frac{\partial g_{i h}}{\partial y_{j h^{\prime}}}=\frac{\alpha_{h h^{\prime}} f_{i j h}\left(\exp y_{j}\right)}{\sum_{j \in \mathcal{N}} f_{i j h}\left(\exp y_{j}\right)}$ on the right side of equation (12), $y_{i h}=g_{i h}(y)$ and $y_{i h}^{\prime}=g_{i h}\left(y^{\prime}\right)$ on its left side, and add $\hat{s}_{h} \equiv \sum_{h^{\prime} \in \mathcal{H}} \alpha_{h h^{\prime}} s_{h^{\prime}}$ on both sides. We then have:

$$
\begin{aligned}
y_{i h}-y_{i h}^{\prime}+\hat{s}_{h} & =\sum_{h^{\prime} \in \mathcal{H}} \alpha_{h h^{\prime}} \sum_{j \in \mathcal{N}} \frac{f_{i j h}\left(\exp \hat{y}_{j}\right)}{\sum_{j \in \mathcal{N}} f_{i j h}\left(\exp \hat{y}_{j}\right)}\left(y_{j h^{\prime}}-y_{j h^{\prime}}^{\prime}+s_{h^{\prime}}\right) \Rightarrow \\
\left|y_{i h}-y_{i h}^{\prime}+\hat{s}_{h}\right| & \leq \sum_{h^{\prime} \in \mathcal{H}}\left|\alpha_{h h^{\prime}}\right| d_{h^{\prime}} \Rightarrow \\
d_{h} & \leq \sum_{h^{\prime} \in \mathcal{H}}\left|\alpha_{h h^{\prime}}\right| d_{h^{\prime}}
\end{aligned}
$$

where the first and second steps are due to the above properties (1) and (3), respectively. Since for some $h, d_{h}>0$, there must exist $h^{\prime}$ such that $d_{h^{\prime}}>0$ and $\alpha_{h h^{\prime}} \neq 0$; thus, applying Property (2) in the above first step, we must have the inequality strictly holds for such $h$. That is: whenever $d_{h}>0, d_{h}<\sum_{h^{\prime} \in \mathcal{H}}\left|\alpha_{h h^{\prime}}\right| d_{h^{\prime}}$. Again, according to the Collatz-Wielandt Formula, we have $\rho(\mathbf{A})>1$, which is a contradiction.

Part iii: Consider $\left\{K_{i j h}>0\right\}_{i, j \in \mathcal{N}, h \in \mathcal{H}}$ which satisfies $\sum_{j \in \mathcal{N}} K_{i j h}=1$ for any $i$. Obviously, 
$x=1$ is one solution of equation (3). In the following, we define $\bar{x} \in \mathbb{R}_{++}^{N \times H}$, which is (columnwise up-to-scale) different from $x$, and show it is also a solution

To define $\bar{x}_{j h^{\prime}}$, we classify its indexes $j h^{\prime}$ into $4 H+1$ sets. First, arbitrarily classify all $j \in \mathcal{N}$ into $2 H+1$ non-empty disjoint sets $\left\{\mathcal{N}_{h}^{+}, \mathcal{N}_{h}^{-}, \mathcal{N}^{0}\right\}_{h \in H}$; second, if $j$ is in $\mathcal{N}_{h}^{+}$ or $\mathcal{N}_{h}^{-}$, we classify $h^{\prime}$ into two sets $\mathcal{H}_{h}^{+} \equiv\left\{h^{\prime} \mid \alpha_{h h^{\prime}}>0\right\}$ and $\mathcal{H}_{h}^{-} \equiv\left\{h^{\prime} \mid \alpha_{h h^{\prime}} \leq 0\right\}\left(\mathcal{H}_{h}^{+}\right.$or $\mathcal{H}_{h}^{-}$may be empty). Let $z \in \mathbb{R}_{+}^{H}$ be an eigenvector of matrix $\mathbf{A}$ such that $\mathbf{A} z=\rho(\mathbf{A}) z$. Now we are ready to define $\bar{x}_{j h^{\prime}}$. If $j \in \mathcal{N}_{h}^{+}, \bar{x}_{j h^{\prime}} \equiv\left\{\begin{array}{ll}\exp \left(+z_{h^{\prime}}\right) & h^{\prime} \in \mathcal{H}_{h}^{+} \\ \exp \left(-z_{h^{\prime}}\right) & h^{\prime} \in \mathcal{H}_{h}^{-}\end{array} ;\right.$if $j \in \mathcal{N}_{h}^{-}$, $\bar{x}_{j h^{\prime}} \equiv\left\{\begin{array}{ll}\exp \left(-z_{h^{\prime}}\right) & h^{\prime} \in \mathcal{H}_{h}^{+} \\ \exp \left(+z_{h^{\prime}}\right) & h^{\prime} \in \mathcal{H}_{h}^{-}\end{array} ;\right.$if $j \in \mathcal{N}^{0}, \bar{x}_{j h^{\prime}} \equiv 1$.

Note that $\prod_{h^{\prime} \in \mathcal{H}} \bar{x}_{j h^{\prime}}^{\alpha_{h h^{\prime}}}$ must be between $\exp \left(-\sum_{h^{\prime}=\mathcal{H}}\left|\alpha_{h h^{\prime}}\right| z_{h^{\prime}}\right)$ and $\exp \left(\sum_{h^{\prime}=\mathcal{H}}\left|\alpha_{h h^{\prime}}\right| z_{h^{\prime}}\right)$. Furthermore, if $j \in \mathcal{N}_{h}^{+}, \prod_{h^{\prime} \in \mathcal{H}} \bar{x}_{j h^{\prime}}^{\alpha_{h h^{\prime}}}=\exp \left(\sum_{h^{\prime}=\mathcal{H}}\left|\alpha_{h h^{\prime}}\right| z_{h^{\prime}}\right)$; if $j \in \mathcal{N}_{h}^{-}, \prod_{h^{\prime} \in \mathcal{H}} \bar{x}_{j h^{\prime}}^{\alpha_{h h^{\prime}}}=$ $\exp \left(-\sum_{h^{\prime}=\mathcal{H}}\left|\alpha_{h h^{\prime}}\right| z_{h^{\prime}}\right)$. Notice that $\sum_{h^{\prime}=\mathcal{H}}\left|\alpha_{h h^{\prime}}\right| z_{h^{\prime}}=\rho(\mathbf{A}) z_{h}$. Thus:

$\sum_{j \in \mathcal{N}} K_{i j h} \prod_{h^{\prime} \in \mathcal{H}} \bar{x}_{j h^{\prime}}^{\alpha_{h h^{\prime}}}=\exp \left(\rho(\mathbf{A}) z_{h}\right) \sum_{j \in \mathcal{N}_{h}^{+}} K_{i j h}+\exp \left(-\rho(\mathbf{A}) z_{h}\right) \sum_{j \in \mathcal{N}_{h}^{-}} K_{i j h}+\sum_{j \notin \mathcal{N}_{h}^{+} \cup \mathcal{N}_{h}^{-}} K_{i j h} \prod_{h^{\prime} \in \mathcal{H}} \bar{x}_{j h^{\prime}}^{\alpha_{h h^{\prime}}}$,

where in the last term $\prod_{h^{\prime} \in \mathcal{H}} \bar{x}_{j h^{\prime}}^{\alpha_{h h^{\prime}}}$ is between $\exp \left(\rho(\mathbf{A}) z_{h}\right)$ and $\exp \left(-\rho(\mathbf{A}) z_{h}\right)$.

If $z_{h}=0$, the above equation is equal to $1\left(=\exp \left(-z_{h}\right)=\exp \left(z_{h}\right)\right)$ since $\sum_{j \in \mathcal{N}} K_{i j h}=$ 1 ; if $z_{h} \neq 0$, we can set it to be any value that is strictly between $\exp \left(\rho(\mathbf{A}) z_{h}\right)$ and $\exp \left(-\rho(\mathbf{A}) z_{h}\right)$ by appropriately choosing $\left\{K_{i j h}>0\right\}_{j \in \mathcal{N}}$ while keeping $\sum_{j \in \mathcal{N}} K_{i j h}=1$. In particular, since $\rho(\mathbf{A})>1$, we can set it to be $\exp \left(-z_{h}\right), 1$, or $\exp \left(z_{h}\right)$, which are the range of $x_{i h}$. Thus we have $\sum_{j \in \mathcal{N}} K_{i j h} \prod_{h^{\prime} \in \mathcal{H}} \bar{x}_{j h^{\prime}}^{\alpha_{h h^{\prime}}}=\bar{x}_{i h}$. That is: $\bar{x}$ is also a solution of equation (1), as desired. 


\title{
On the Equilibrium Properties of Network Models with Heterogeneous Agents: Online Appendix
}

\author{
Treb Allen, Costas Arkolakis and Xiangliang Li
}

\section{Table of Contents}

A Additional Applications $\quad 1$

A.1 Discrete choice over many actions with social interactions . . . . . . . 1

A.2 Choosing many (continuous) actions with social interactions . . . . . . . 2

A.3 Public goods in social networks f. . . . . . . . . . . 3

A.4 Multiple activities in social networks . . . . . . . . . . . . . 4

A.5 An urban model with spatial spillovers . . . . . . . . . . . 5

A.6 An economic geography model with spatial spillovers . . . . . . . . . 8

A.7 A trade model with intermediate goods and tariffs . . . . . . . . . . 10

A.8 A production network with multiple intermediates goods . . . . . . . . 14

A.9 Productivity identification in a production network with many locations

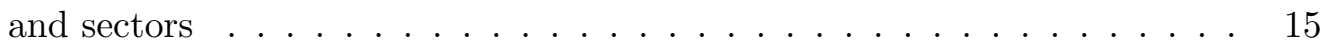

A.10 Inverting a demand system with multiple types of goods . . . . . . . . 17

B Additional Details $\quad 19$

B.1 Details of Remarks . . . . . . . . . . . . . . . . . . 19

B.2 Details of the Urban Spatial Model . . . . . . . . . . . . . . 23 


\section{A Additional Applications}

We apply Theorem 1 to ten additional applications in the fields of social networks, spatial networks, production networks, and demand estimation.

\section{A.1 Discrete choice over many actions with social interactions}

Here we consider a discrete choice framework with social interactions as in the seminal paper of Brock and Durlauf (2001), generalized to include agents simultaneously choosing over many types of actions with flexible social spillovers across agents and actions.

\section{A.1.1 The model}

Setup Suppose there are $N$ agents. Each agent $i \in\{1, \ldots, N\} \equiv \mathcal{N}$ makes a discrete choice over $\mathcal{H} \equiv\{1, \ldots, H\}$, a set of $H$ actions.

$$
V_{i h}\left(\mu_{i}\right)=u_{i h}+S_{i h}\left(\mu_{i}\right)+\varepsilon_{i h}
$$

where $u_{i h}$ is the private utility associated with action $h, S_{i h}\left(\mu_{i}\right)$ is the social utility, $\mu_{i}$ is agent $i$ 's belief of other agents' actions, and $\varepsilon_{i h}$ is a random utility term, independently and identically distributed across agents and actions. Agent chooses action

$$
\arg \max _{h \in \mathcal{H}} V_{i h}\left(\mu_{i}\right)
$$

that maximizes her payoffs given her belief of the actions of others. Define $\mu_{i j h}$ to be the conditional probability measure agent $i$ places on the probability that agent $j$ chooses action $h$. We assume that $S_{i h}\left(\mu_{i}\right)$ takes the following form:

$$
S_{i h}\left(\mu_{i}\right)=\sum_{h^{\prime} \in \mathcal{H}} J_{h h^{\prime}} \ln \left(\bar{\mu}_{i h^{\prime}}\right)
$$

where $J_{h h^{\prime}}$ reflects the impact of action $h^{\prime}$ by others on agent $i$ 's utility when she chooses action $h, \bar{\mu}_{i h^{\prime}} \equiv \sum_{j \neq i} w_{i j h^{\prime}} \mu_{i j h^{\prime}}$ is her (weighted) expected number of agents taking action $h^{\prime}, w_{i j h^{\prime}}$ is the corresponding weight, and $\mu_{i j h^{\prime}}$ is her belief of agent $i$ taking action $h^{\prime}$. We note that the log transform on the social utility function - not present in the primary case considered by Brock and Durlauf (2001) - ensures that the uniqueness of the equilibrium can be characterized without reference to an (endogenous) threshold value (c.f. Brock and Durlauf (2001) Proposition 2).

Equilibrium Retaining the assumption from Brock and Durlauf (2001) that the random utility term follows a type I extreme value distribution with shape parameter $\beta_{h}$ and agent's conditional probabilities are rational (so that $\mu_{i j h}=\mu_{j h}$ for all $j \in\{1, \ldots, N\}$ and $\mu_{j h}$ is equal to the probability agent $j$ actually chooses action $h$ ) results in the following equilibrium conditions for all $i \in\{1, \ldots, N\}$ and for all $h \in\{1, \ldots, H\}$ :

$$
\mu_{i h}=\frac{e^{\beta_{h} u_{i h}}\left(\Pi_{h^{\prime} \in \mathcal{H}}\left(\sum_{j \neq i} w_{i j h^{\prime}} \mu_{j h^{\prime}}\right)^{\beta_{h} J_{h h^{\prime}}}\right)}{\sum_{k \in \mathcal{H}} e^{\beta_{k} u_{i k}}\left(\Pi_{h^{\prime} \in \mathcal{H}}\left(\sum_{j \neq i} w_{i j h^{\prime}} \mu_{j h^{\prime}}\right)^{\beta_{k} J_{k h^{\prime}}}\right)}
$$


Note this is a system of $N \times H$ equilibrium conditions in $N \times H$ unknown probabilities $\mu_{j h}$. Equation (16) is a special case of (1). From Remark 5, define $x_{i h} \equiv \sum_{j \neq i} w_{i j h} \mu_{j h}$, which, when combined with equation (16), becomes:

$$
x_{i h}=\sum_{j \neq i} w_{i j h} \frac{e^{\beta_{h} u_{j h}} \Pi_{h^{\prime} \in \mathcal{H}}\left(x_{j h^{\prime}}\right)^{\beta_{h} J_{h h^{\prime}}}}{\left(\sum_{k \in \mathcal{H}} e^{\beta_{k} u_{j k}}\left(\Pi_{h^{\prime} \in \mathcal{H}}\left(x_{j h^{\prime}}\right)^{\beta_{k} J_{k h^{\prime}}}\right)\right)}
$$

Finally, defining $f_{i j h} \equiv w_{i j h} \frac{e^{\beta_{h} u_{j h}} \Pi_{h^{\prime} \in \mathcal{H}}\left(x_{j h^{\prime}}\right)^{\beta_{h} J_{h h^{\prime}}}}{\left(\sum_{k \in \mathcal{H}} e^{\beta_{k} u_{j k}}\left(\Pi_{h^{\prime} \in \mathcal{H}}\left(x_{j h^{\prime}}\right)^{\beta_{k} J_{k h^{\prime}}}\right)\right)}$ if $j \neq i$ and $f_{i i h}=0$ results in equation (17) be written as:

$$
x_{i h}=\sum_{j \in \mathcal{N}} f_{i j h}\left(x_{j 1}, \ldots, x_{j H}\right),
$$

as in (1).

\section{A.1.2 Applying Theorem 1}

It is straightforward to calculate the elasticities of interactions as follows:

$$
\frac{\partial \ln f_{i j, h}}{\partial \ln x_{j, h^{\prime}}}=\beta_{h} J_{h h^{\prime}}-\sum_{k} \frac{e^{\beta_{k} u_{j k}}\left(\Pi_{h^{\prime} \in \mathcal{H}}\left(x_{j h^{\prime}}\right)^{\beta_{k} J_{k h^{\prime}}}\right)}{\sum_{k \in \mathcal{H}} e^{\beta_{k} u_{j k}}\left(\Pi_{h^{\prime} \in \mathcal{H}}\left(x_{j h^{\prime}}\right)^{\beta_{k} J_{k h^{\prime}}}\right)} \beta_{k} J_{k h^{\prime}},
$$

which is between $\underline{J}_{h h^{\prime}} \equiv \beta_{h} J_{h h^{\prime}}-\max _{k \in \mathcal{H}} \beta_{k} J_{k h^{\prime}}$ and $\bar{J}_{h h^{\prime}} \equiv \beta_{h} J_{h h^{\prime}}-\min _{k \in \mathcal{H}} \beta_{k} J_{k h^{\prime}}$. Thus if we define:

$$
(\mathbf{A})_{h h^{\prime}} \equiv \max \left(-\underline{J}_{h h^{\prime}}, \bar{J}_{h h^{\prime}}\right)
$$

then we have for all $h, h^{\prime}$ :

$$
\left|\frac{\partial \ln f_{i j, h}}{\partial \ln x_{j, h^{\prime}}}\right| \leq(\mathbf{A})_{h h^{\prime}}
$$

From Theorem 1, there is a unique solution if $\rho(\mathbf{A})<1$, i.e. as long as the social spillovers are not too heterogeneous across actions.

\section{A.2 Choosing many (continuous) actions with social interactions}

Here we consider a framework with non-market interactions as in Glaeser and Scheinkman (2002), generalized to include many actions and a general network structure.

\section{A.2.1 The Model}

Setup Suppose there are $N$ agents where each agent $i \in\{1, \ldots, N\} \equiv \mathcal{N}$ who chooses actions $\left\{x_{i h}\right\}$, indexed by $h \in\{1, \ldots, H\} \equiv \mathcal{H}$. Let agent $i^{\prime} s$ payoffs depend on her own actions and the actions of others as follows:

$$
U_{i}\left(\left\{x_{i h}\right\}_{h \in \mathcal{H}} ;\left\{\sum_{j \neq i} g_{i j h^{\prime}} x_{j h^{\prime}}\right\}_{h^{\prime} \in \mathcal{H}}\right)
$$


where the utility function $U_{i}$ is strictly concave in each $x_{i h}, g_{i j h^{\prime}} \geq 0$, and $\sum_{j \neq i} g_{i j h^{\prime}} x_{j h^{\prime}}$ measures the aggregate behavior of agent $i$ 's peers. Note that this generalizes Glaeser and Scheinkman (2002) to include an unrestricted network structure $\left\{g_{i j h^{\prime}}\right\}$ and arbitrary $H$.

Equilibrium Suppose there exists a unique solution to the utility maximization problem of equation (18) that can be written as:

$$
x_{i h}=f_{i h}\left(\left\{\sum_{j \neq i} g_{i j h^{\prime}} x_{j h^{\prime}}\right\}_{h^{\prime} \in \mathcal{H}}\right),
$$

where $f_{i h}$ is the best response function. Following Remark 5, we define $y_{i h} \equiv \sum_{j \neq i} g_{i j h} x_{j h}$ and substitute the expression (19), yielding:

$$
y_{i h}=\sum_{j \neq i} g_{i j h} f_{j h}\left(\left\{y_{j h^{\prime}}\right\}_{h^{\prime} \in \mathcal{H}}\right) .
$$

\section{A.2.2 Applying Theorem 1}

It is immediately evident that equation (20) is a special case of equation (1). Suppose that the elasticities of the spillover function can be bounded, i.e. for all $h, h^{\prime} \in \mathcal{H}$ there exists an $\alpha_{h h^{\prime}} \geq 0$ such that $\left|\frac{\partial \ln f_{j h}}{\partial \ln y_{j h^{\prime}}}\right| \leq \alpha_{h h^{\prime}}$ for all $\left\{y_{j h^{\prime}}\right\}_{h^{\prime} \in \mathcal{H}}$. Let $\mathbf{A}$ be the $H \times H$ matrix whose $\left(h, h^{\prime}\right)$ element is $\alpha_{h h^{\prime}}$. From Theorem 1 part (i), there exists a unique equilibrium if $\rho(\mathbf{A})<1$. Moreover, that equilibrium can be reached from any initial starting point $\left\{y_{j h^{\prime}}^{0}\right\}_{j \in \mathcal{N}}^{h^{\prime} \in \mathcal{H}}$ by iteration of equation (20).

Glaeser and Scheinkman (2002) prove uniqueness in the $H=1$ case where $\sum_{j \neq i} g_{i j}=1$ if a "Moderate Social Influence" condition holds, i.e. $\left|\frac{\partial f_{j}}{\partial y_{j}}\right|<1$ for all $y_{j}$. Notice that their results are actually implied by Remark 3 and depend on the particular network structure. In the $H=1$ case, our condition, obtained from Theorem 1, simplifies to $\left|\frac{\partial \ln f_{j}}{\partial \ln y_{j}}\right|<1$ for all $y_{j}$, regardless of the structure of $\left\{g_{i j}\right\}$. More generally, ours is the first characterization (of which we are aware) for the $H>1$ case with general $\left\{g_{i j h}\right\}$.

\section{A.3 Public goods in social networks}

Here we consider a framework where agents decide how much of their own resources to contribute to public goods whose payoff depends on the contributions of others. To do so, we extend the work of Allouch (2015) and Acemoglu, García-Jimeno, and Robinson (2015) to allow agents to contribute multiple types of public goods on multiple social networks. ${ }^{12}$

\section{A.3.1 The model}

Setup Consider a world of $i \in\{1, \ldots, N\} \equiv \mathcal{N}$ agents. Agent $i \in \mathcal{N}$ is endowed with wealth $w_{i}$ and chooses how allocate that wealth to private consumption $\left(c_{i}\right)$ or contributions to $H$ public

\footnotetext{
${ }^{12}$ Allouch (2015) and Acemoglu, García-Jimeno, and Robinson (2015) extend the work of Bramoullé, Kranton, and D'amours (2014), who applies the seminal work of the private provision of public goods in Bergstrom, Blume, and Varian (1986) to a network setting, but with non-linear best response functions.
} 
goods $\left(\left\{q_{i h}\right\}_{h \in\{1,2,3 \ldots H\} \equiv \mathcal{H}}\right)$, where her payoff depends on the contributions of all other agents. In particular, agent $i \in \mathcal{N}$ solves:

$$
\begin{gathered}
\max _{c_{i}, q_{i}} U_{i}\left(c_{i},\left\{Q_{i h}\right\}_{h}\right) \\
\text { s.t. } c_{i}+\sum_{h} q_{i h}=w_{i}+\sum_{h} Q_{-i h} \text { and } q_{i} \geq 0,
\end{gathered}
$$

where $U_{i}(\cdot)$ is the utility function $Q_{i h}=q_{i h}+Q_{-i h}$ is the public good bundle with $Q_{-i h}=$ $\sum_{j \neq i} g_{i j h} q_{j h}$ the contributions of all other agents, and the $N \times N$ matrix $\mathbf{G}_{h} \equiv\left[g_{i j h}\right]$ governs the payoff of $j$ 's contribution of public good $h$ to agent $i$ (thereby defining the social network $h$ ).

Equilibrium Suppose that $U_{i}(\cdot)$ is continuous, strictly increasing in all its arguments, and strictly quasi-concave. Solving agent's utility maximization problem gives rise to agent $i$ demand function of public good $h, \gamma_{i h}\left(w_{i}+\sum_{h^{\prime}} Q_{-i h^{\prime}}\right)$. Notice that if this demand function is less than other agents' contribution $Q_{-i h}$, agent $i$ will contribute nothing in public goods. That is in equilibrium we have:

$$
q_{i h}=\max \left(\gamma_{i h}\left(w_{i}+\sum_{h^{\prime}} Q_{-i h^{\prime}}\right)-Q_{-i h}, 0\right) .
$$

We assume that $\gamma_{i h}(\cdot)$ is differentiable and the private and public goods are all normal goods i.e. $0<\gamma_{i h}^{\prime}<1$.

\section{A.3.2 Applying Theorem 1's Remark 3}

Denote the rightside of equation $(21)$ as $F_{i h}(\cdot)$. The right and left partial derivatives of $F_{i h}(\cdot)$ with respect to $q_{j h^{\prime}}$ are either 0 or $\gamma_{i h}^{\prime} g_{i j h^{\prime}}$ if $h \neq h^{\prime}$ and $\left(\gamma_{i h}^{\prime}-1\right) g_{i j h}$ if $h=h^{\prime}$. Thus, according to Remark 3, equation (21) has a unique solution if there exists an $N$-by- $N$ matrix B satisfying $\rho(\mathbf{B})<1$ such that for all $i, j \in \mathcal{N}, h \in \mathcal{H}, \sum_{h^{\prime}} \max \left(\left|\frac{\partial_{+} F_{i h}(x)}{\partial x_{j h^{\prime}}}\right|,\left|\frac{\partial_{-} F_{i h}(x)}{\partial x_{j h^{\prime}}}\right|\right) \leq\left|\gamma_{i h}^{\prime} \sum_{h^{\prime}} g_{i j h^{\prime}}-g_{i j h}\right| \leq(\mathbf{B})_{i j}$. Intuitively, as long as the aggregate spillovers between agents' public goods contributions are not too strong, the incentives of any agent to shirk her contribution to public goods are not large enough to result in multiplicity. When $H=1$, this condition can be reduced to $\rho(G)<\frac{1}{1-\gamma_{i}^{\prime}}$ where $G$ represents the only network. This condition is very similar to (and stronger than) Allouch (2015)'s $-\lambda_{\min }(G)<\frac{1}{1-\gamma_{i}^{\prime}}$, since $-\lambda_{\min }(G) \leq \rho(G)$. However, the well-definedness of $\lambda_{\min }(G)$ crucially relies on network $G$ being symmetric (the symmetry guarantees all the eigenvalues of $G$ are real and can be ranked); in contrast, the condition provided here is valid for asymmetric networks as well.

\section{A.4 Multiple activities in social networks}

We extend the work of Chen, Zenou, and Zhou (2018) where agent's payoffs depend on their own multiple actions as well as the actions of others in their social networks to more than two types of actions on multiple social networks. Unlike in Section 3.2, here we focus on linear best response functions in order to extend the domain of solutions to include zero and negative values.

\section{A.4.1 The model}

Setup Consider a system of $h \in\{1,2,3 \ldots H\} \equiv \mathcal{H}$ social networks with $N$ agents. Each agent $i \in\{1,2,3 \ldots N\} \equiv \mathcal{N}$ has preferences over actions $\left\{x_{i h}\right\}_{h \in \mathcal{H}}$ which take real numbers. We assume 
that agents' preferences are represented by the quadratic utility function:

$$
U_{i}=\sum_{h=1}^{H}\left(c_{i h} x_{i h}-\frac{1}{2} x_{i h}^{2}\right)+\sum_{h=1}^{H} \sum_{h^{\prime}=1}^{H} d_{i h h^{\prime}} x_{i h} x_{i h^{\prime}}+\sum_{h=1}^{H} \sum_{j=1}^{N} g_{i j h} x_{i h} x_{j h},
$$

where $c_{i h}, d_{i h h^{\prime}}$, and $g_{i j h}$ are exogenously given constants and for all $i, h, d_{i h h}=0$ and $g_{i i h}=0$. The first term in the above expression, reflects decreasing returns to scale of agent $i$ 's own actions; the second term reflects substitution or complementary effects between agent $i$ 's different actions; the last term reflects the network externality from other agents and $\left\{g_{i j h}\right\}_{i, j \in \mathcal{N}}$ represents the corresponding social network.

Equilibrium We assume the above utility function is concave. Thus its maximum can be characterized by the first order condition:

$$
x_{i h}=c_{i h}+\sum_{h^{\prime} \neq h}\left(d_{i h h^{\prime}}+d_{i h^{\prime} h}\right) x_{i h^{\prime}}+\sum_{j \neq i} g_{i j h} x_{j h} .
$$

Define the $H$-by- $H$ symmetric matrix $D_{i}$ such that $\left(D_{i}\right)_{h h^{\prime}}=d_{i h h^{\prime}}+d_{i h h^{\prime}}$. The concavity assumption implies that matrix $I-D_{i}$ is positive definite and thus invertible. Denote the element of the inverse of $I-D_{i}$ as $\delta_{i h h^{\prime}}$. Then we can rewrite the above first order condition as:

$$
x_{i h}=\sum_{h^{\prime}} \delta_{i h h^{\prime}}\left(c_{i h^{\prime}}+\sum_{j \neq i} g_{i j h^{\prime}} x_{j h^{\prime}}\right) .
$$

This equation then represents the Nash equilibrium of this network game.

\section{A.4.2 Applying Theorem 1's Remark 3}

Denote the rightside of equation 22 as $F_{i h}(\cdot)$. Notice that $\frac{\partial F_{i h}(x)}{\partial x_{j h^{\prime}}}=\delta_{i h h^{\prime}} g_{i j h^{\prime}}$. Thus, according to Remark 3, equation (22) has a unique solution if there exists an $N$-by- $N$ matrix B satisfying $\rho(\mathbf{B})<1$ such that for all $i, j \in \mathcal{N}, h \in \mathcal{H}, \sum_{h^{\prime}}\left|\delta_{i h h^{\prime}} g_{i j h^{\prime}}\right| \leq(\mathbf{B})_{i j}$. Intuitively, if the aggregate connections of different networks are low, agents' influences on each other are weak enough such that the multiplicity as in coordination games then is excluded.

We note that our condition simplifies to the one given by Chen, Zenou, and Zhou (2018) in the special case considered there of $H=2, d_{i h h^{\prime}}+d_{i h h^{\prime}}=-\beta\left(h=1, h^{\prime}=2\right)$, and for all $i, j$, $g_{i j 1}=g_{i j 2}=g_{i j}$ i.e. there is a single network $G$. To see this, note that by calculating the inverse of $I-D_{i}$, we have $\sum_{h^{\prime}}\left|\delta_{i h h^{\prime}} g_{i j h^{\prime}}\right|=\frac{1}{1-|\beta|} g_{i j}$. Then our condition can be written as $\rho(G)<1-|\beta|$, which is the one used in Chen, Zenou, and Zhou (2018).

\section{A.5 An urban model with spatial spillovers}

Here we consider another variant of the urban spatial model based on the seminal work of Ahlfeldt, Redding, Sturm, and Wolf (2015) presented in Section 3.1, where we include productivity and amenity spillovers that depend flexibly on the distribution of workers and residents, respectively, across the entire city. 


\section{A.5.1 The Model}

We first describe the model and derive its equilibrium conditions.

Setup Consider a city comprised of $i \in\{1, \ldots, N\} \equiv \mathcal{N}$ blocks inhabited by agents with measure $\bar{L}$. Each agent $\nu$ chooses where to live $i \in \mathcal{N}$ and where to work $j \in \mathcal{N}$ in order to maximize her utility:

$$
U_{i j}(\nu)=\frac{u_{i} w_{j}}{\mu_{i j}} \varepsilon_{i j}(\nu),
$$

where $u_{i}$ and $w_{j}$ are the value of living at block $i$ and working at block $j$, respectively, common to all agents, $\mu_{i j} \geq 1$ is the commuting cost, and $\varepsilon_{i j}(\nu)$ is the idiosyncratic preference of agent $\nu$ over location pairs, which we assume is extreme value (Frechet) distributed with shape parameter $\theta>0$.

Commuting flows The number of agents who choose to live in location $i$ and work in location $j$ can be written as:

$$
L_{i j}=\left(\frac{u_{i} w_{j}}{\mu_{i j}}\right)^{\theta} \lambda,
$$

where $\lambda \equiv \bar{L} W^{-\theta_{h}}$ and $W \equiv\left(\sum_{(i, j) \in \mathcal{N}^{2}}\left(\frac{u_{i} w_{j}}{\mu_{i j}}\right)^{\theta}\right)^{\frac{1}{\theta}}=\mathbb{E}\left(\max _{(i, j) \in \mathcal{N}^{2}} U_{i j}(\nu)\right)$ is the expected welfare of agents.

Spatial Spillovers Suppose that an agent working in block $j$ produces a costlessly traded numeraire good, for which they are paid their marginal product $A_{j}$, which is the only value they derive from their work, i.e. $w_{j}=A_{j}$. Suppose that their productivity depends both on the innate productivity of block $j, \bar{A}_{j}$, and the entire distribution of populations of workers throughout the city as follows:

$$
A_{i}=\bar{A}_{i}\left(\sum_{j \in \mathcal{N}} F_{i j}^{A} L_{j}^{F}\right)^{\alpha},
$$

where $F_{i j}^{A}>0$ governs the effect of the number of workers in $j \in \mathcal{N}$ on the productivity of a worker in $i \in \mathcal{N}$ and $\alpha$ governs the overall strength of the productivity spillover.

Similarly, suppose that an agent residing in block $i$ receives a value of living there that depends both on the innate amenity of block $i, \bar{u}_{i}$, and the entire distribution of populations of residents throughout the city as follows:

$$
u_{i}=\bar{u}_{i}\left(\sum_{j \in \mathcal{N}} F_{i j}^{u} L_{j}^{R}\right)^{\beta},
$$

where $F_{i j}^{u}>0$ governs the effect of the number of residents in $j \in \mathcal{N}$ on the amenity of a worker in $i \in \mathcal{N}$ and $\beta$ governs the overall strength of the amenity spillover. ${ }^{13}$

\footnotetext{
${ }^{13}$ Assuming alternative spillover functions $A_{i}=\bar{A}_{i} \sum_{j \in \mathcal{N}} F_{i j}^{A}\left(L_{j}^{F}\right)^{\alpha}$ and $u_{i}=\bar{u}_{i} \sum_{j \in \mathcal{N}} F_{i j}^{u}\left(L_{j}^{R}\right)^{\beta}$ result in an elasticity matrix with the same spectral radius as the one below, i.e. the conclusions of Theorem 1 below are unchanged.
} 
Equilibrium For any geography $\left\{\left\{\mu_{i j}, F_{i j}^{A}, F_{i j}^{u}\right\}_{(i, j) \in \mathcal{N}^{2}},\left\{\bar{A}_{i}, \bar{u}_{i}\right\}_{i \in \mathcal{N}}\right\}$, measure of agents $\bar{L}$, and model elasticities $\{\theta, \alpha, \beta\}$, equilibrium is a set of workplace and residential populations $\left\{L_{i}^{F}, L_{i}^{R}\right\}_{i \in \mathcal{N}}$ such that:

1. The measure of workers employed in block $i \in \mathcal{N}$ is equal to the total number of agents commuting to that location:

$$
L_{i}^{F}=\sum_{j \in \mathcal{N}} L_{j i}
$$

2. The measure of residents residing in block $i \in \mathcal{N}$ is equal to the total number of agents commuting from that location:

$$
L_{i}^{R}=\sum_{j \in \mathcal{N}} L_{i j}
$$

As in Section 3.1 (and unlike Ahlfeldt, Redding, Sturm, and Wolf (2015)) we do not impose that rental rates of residential and commercial floor spaces are equalized.

\section{A.5.2 Applying Theorem 1}

Substituting the commuting equation (24) into the equilibrium conditions (27) and (28) and rearranging equations (25) and (26) yields:

$$
\begin{gathered}
L_{i}^{F} A_{i}^{-\theta}=\lambda \sum_{j \in \mathcal{N}} \mu_{j i}^{-\theta} u_{j}^{\theta} \\
L_{i}^{R} u_{i}^{-\theta}=\lambda \sum_{j \in \mathcal{N}} \mu_{i j}^{-\theta} A_{j}^{\theta}, \\
A_{i}^{\frac{1}{\alpha}}=\bar{A}_{i}^{\frac{1}{\alpha}} \sum_{j \in \mathcal{N}} F_{i j}^{A} L_{j}^{F} \\
u_{i}^{\frac{1}{\beta}}=\bar{u}_{i} \sum_{j \in \mathcal{N}} F_{i j}^{u} L_{j}^{R},
\end{gathered}
$$

which together comprise our equilibrium system. It is immediately evident that this system of $4 N$ equations in $4 N$ unknowns takes the form of equation (3), which is a special case of equation (1), so by applying Remarks 4 and 5, it is sufficient to characterize the spectral radius of matrix $\mathbf{A} \equiv\left|\mathbf{B} \boldsymbol{\Gamma}^{-1}\right|$, where:

$$
\mathbf{B} \equiv\left(\begin{array}{cccc}
0 & 0 & 0 & \theta \\
0 & 0 & \theta & 0 \\
1 & 0 & 0 & 0 \\
0 & 1 & 0 & 0
\end{array}\right), \quad \boldsymbol{\Gamma} \equiv\left(\begin{array}{cccc}
1 & 0 & -\theta & 0 \\
0 & 1 & 0 & -\theta \\
0 & 0 & \frac{1}{\alpha} & 0 \\
0 & 0 & 0 & \frac{1}{\beta}
\end{array}\right)
$$

so that:

$$
\mathbf{A} \equiv\left(\begin{array}{cccc}
0 & 0 & 0 & |\beta \theta| \\
0 & 0 & |\alpha \theta| & 0 \\
1 & 0 & |\alpha \theta| & 0 \\
0 & 1 & 0 & |\beta \theta|
\end{array}\right)
$$

From Remark 7, a sufficient condition for uniqueness is hence $|\alpha| \theta \leq \frac{1}{2}$ and $|\beta| \theta \leq \frac{1}{2}$, i.e. both the productivity and amenity agglomeration forces must be no stronger than the dispersion forces aris- 
ing from the heterogeneity in agent preferences governed by $\theta$. Note these conditions are identical to the $H=1$ case of the example presented in Section 3.1, i.e. the presence of spatial spillovers does not affect the uniqueness condition.

We remark that while the full model presented in Ahlfeldt, Redding, Sturm, and Wolf (2015) included spatial spillovers, that paper only offered conditions for uniqueness in the absence of such spillovers; as a result, to our knowledge this is the first proof of uniqueness of an urban model in the presence of spatial spillovers. A similar methodology can be applied to incorporate spatial spillovers in other spatial settings - but with very different implications for the properties of the model - as we illustrate in the following economic geography example.

\section{A.6 An economic geography model with spatial spillovers}

We now extend the economic geography framework of Allen and Arkolakis (2014) to incorporate spatial productivity and amenity spillovers. It turns out that any spatial productivity or amenity spillovers can result in multiple equilibria - a very different conclusion from the urban model-highlighting the importance of Theorem 1 part (iii).

\section{A.6.1 The model}

Setup There are $N$ locations, each of which produces a differentiated variety of a good. Agents in location $i \in\{1, \ldots, N\} \equiv \mathcal{N}$ have constant elasticity of substitution preferences over the differentiated varieties so that their welfare $W_{i}$ is:

$$
W_{i}=\left(\sum_{j \in \mathcal{N}} q_{j i}^{\frac{\sigma-1}{\sigma}}\right)^{\frac{\sigma}{\sigma-1}} u_{i}
$$

where $q_{j i}$ is the quantity of goods produced in $j \in \mathcal{N}$ and consumed in $i, \sigma \geq 1$ is the elasticity of substitution, and $u_{i}$ is the local amenity. Agents are perfectly mobile and earn wage $w_{i}$ by supplying their unit labor inelastically. Labor is the only factor of production; let $A_{i}$ be the productivity of an agent in location $i \in \mathcal{N}$. Finally, the transportation of goods are subject to iceberg transportation costs, where $T_{i j} \geq 1$ indicates the number of goods needed to be sent from $i \in \mathcal{N}$ in order for one unit to arrive in $j \in \mathcal{N}$.

Spatial spillovers We suppose that productivities and amenities depend on the distribution of labor across all locations through spatial spillovers as follows:

$$
\begin{aligned}
A_{i} & =\bar{A}_{i}\left(\sum_{j \in \mathcal{N}} F_{i j}^{A} L_{j}\right)^{\alpha} \\
u_{i} & =\bar{u}_{i}\left(\sum_{j \in \mathcal{N}} F_{i j}^{u} L_{j}\right)^{\beta}
\end{aligned}
$$

where $\bar{A}_{i}$ and $\bar{u}_{i}$ are the exogenous productivity and amenity, respectively, of location $i \in \mathcal{N}$; $F_{i j}^{A}>0$ and $F_{i j}^{u}>0$ capture how the population in location $j \in \mathcal{N}$ affects the productivity and amenity, respectively in location $i \in \mathcal{N}$, and $\alpha$ and $\beta$ are the productivity and amenity spillover 
elasticities, respectively common to all locations. ${ }^{14}$

Equilibrium For any geography $\left\{\left\{T_{i j}\right\}_{(i, j) \in \mathcal{N}^{2}},\left\{\bar{A}_{i}, \bar{u}_{i}\right\}_{i \in \mathcal{N}},\left\{F_{i j}^{A}\right\}_{(i, j) \in N^{2}}\right\}$ equilibrium is a set of populations, wages, productivities, and amenities $\left\{L_{i}, w_{i}, A_{i}, u_{i}\right\}_{i \in \mathcal{N}}$ such that:

1. Markets clear, i.e. income in a location $i \in \mathcal{N}$ is equal to the value of all goods sold in all other locations:

$$
w_{i} L_{i}=\sum_{j \in \mathcal{N}} X_{i j}
$$

where $X_{i j}=\frac{T_{i j}^{1-\sigma}\left(w_{i} / A_{i}\right)^{1-\sigma}}{\sum_{k=1}^{N} T_{k j}^{1-\sigma}\left(w_{k} / A_{k}\right)^{1-\sigma}} w_{j} L_{j}$ is the bilateral flow of goods from $i \in \mathcal{N}$ to $j \in \mathcal{N}$.

2. Trade is balanced, i.e. income in a location $i \in \mathcal{N}$ is equal to the value of all goods purchased from all other locations:

$$
w_{i} L_{i}=\sum_{j \in \mathcal{N}} X_{j i}
$$

3. Welfare is equalized, i.e. there exists a scalar $W>0$ such that for all $i \in \mathcal{N}, W_{i} \leq W$, with the equality strict if $L_{i}>0$.

4. Productivities and amenities are given by equations (29) and (30).

\section{A.6.2 Applying Theorem 1}

Combining the first three equilibrium conditions (see equations (10) and (11) of Allen and Arkolakis (2014)) and re-arranging equations (29) and (30) yields the following system of $4 N$ equilibrium conditions in $4 N$ unknowns:

$$
\begin{aligned}
L_{i} w_{i}^{\sigma} A_{i}^{1-\sigma} & =W^{1-\sigma} \sum_{j=1}^{N} T_{i j}^{1-\sigma} L_{j} w_{j}^{\sigma} u_{j}^{\sigma-1} \\
w_{i}^{1-\sigma} u_{i}^{1-\sigma} & =W^{1-\sigma} \sum_{j=1}^{N} T_{j i}^{1-\sigma} w_{j}^{1-\sigma} A_{j}^{\sigma-1} \\
A_{i}^{\frac{1}{\alpha}} & =\bar{A}_{i}^{\frac{1}{\alpha}} \sum_{j \in \mathcal{N}} F_{i j}^{A} L_{j} \\
u_{i}^{\frac{1}{\beta}} & =\bar{u}_{i}^{\frac{1}{\beta}} \sum_{j \in \mathcal{N}} F_{i j}^{u} L_{j}
\end{aligned}
$$

which together comprise our equilibrium system. It is immediately evident that this system takes the form of equation (3), which is a special case of equation (1), so by applying Remarks 4 and 5 ,

\footnotetext{
${ }^{14}$ Assuming alternative spillover functions $A_{i}=\bar{A}_{i} \sum_{j \in \mathcal{N}} F_{i j}^{A} L_{j}^{\alpha}$ and $u_{i}=\bar{u}_{i} \sum_{j \in \mathcal{N}} F_{i j}^{u} L_{j}^{\beta}$ result in an elasticity matrix with the same spectral radius as the one below, i.e. the conclusions of Theorem 1 below are unchanged.
} 
it is sufficient to characterize the spectral radius of matrix $\mathbf{A} \equiv\left|\mathbf{B} \boldsymbol{\Gamma}^{-1}\right|$, where:

$$
\mathbf{B} \equiv\left(\begin{array}{cccc}
1 & \sigma & 0 & \sigma-1 \\
0 & 1-\sigma & \sigma-1 & 0 \\
1 & 0 & 0 & 0 \\
1 & 0 & 0 & 0
\end{array}\right), \boldsymbol{\Gamma} \equiv\left(\begin{array}{cccc}
1 & \sigma & 1-\sigma & 0 \\
0 & 1-\sigma & 0 & 1-\sigma \\
0 & 0 & \frac{1}{\alpha} & 0 \\
0 & 0 & 0 & \frac{1}{\beta}
\end{array}\right)
$$

so that:

$$
\mathbf{A} \equiv\left(\begin{array}{cccc}
1 & 0 & |\alpha|(\sigma-1) & |\beta|(\sigma-1) \\
0 & 1 & |\alpha|(\sigma-1) & |\beta|(\sigma-1) \\
1 & \frac{\sigma}{\sigma-1} & |\alpha|(\sigma-1) & |\beta| \sigma \\
1 & \frac{\sigma}{\sigma-1} & |\alpha|(\sigma-1) & |\beta| \sigma
\end{array}\right)
$$

It can be shown that $\rho(\mathbf{A}) \leq 1$ only if $\alpha=\beta=0$, i.e. only if there are no spatial spillovers. Note that this is a substantial departure from Allen and Arkolakis (2014) and Allen, Arkolakis, and Takahashi (2020), who show that uniqueness is guaranteed in an economic geography model with local spillovers as long as the dispersion forces are stronger than agglomeration forces; in contrast, Thereom 1 part (iii) says that there will be geographies for which there are multiple equilibria for in the presence of any spatial spillover, i.e. for any non-zero $\alpha$ and $\beta$. Note too that this is also a major qualitative difference with the urban example above, where the conditions for uniqueness were the same for local and spatial spillovers.

A simple example suffices to provide intuition for the possibility of multiple equilibria. Consider a world of two identical locations (i.e. $\bar{A}_{i}=\bar{u}_{i}=1$ for $i, j \in\{1,2\}$ ) separated by trade $\operatorname{costs} \tau>1$. Suppose there are only productivity spillovers (i.e. $\beta=0$ ); the case with amenity spillovers is similar. For any $\alpha>0$ and $F_{i j}^{A}=\left\{\begin{array}{ll}1 & \text { if } i=j \\ 0 & \text { if } i \neq j\end{array}\right.$ - i.e. a case where the spillovers are positive and depend only on one's own population - there exists a $\tau>1$ such that there are three equilibria: one in which both locations have an equal population and one in which one of the two locations has a greater concentration of population (to take advantage of the agglomeration forces). But for any $\alpha<0$ and $F_{i j}^{A}=\left\{\begin{array}{ll}0 & \text { if } i=j \\ 1 & \text { if } i \neq j\end{array}\right.$ - i.e. a case where the spillovers are negative and depend only on the other location's population - there exists a $\tau>1$ such that there are again three equilibria: one in which both locations have an equal population and one in which one of the two locations has a greater concentration of population (to take advantage of the fact that the smaller population in the neighbor increases productivity spillovers). That is, with spatial spillovers, a dispersion force from population elsewhere acts like a local agglomeration force.

To our knowledge, this is the first characterization of uniqueness in an economic geography model with spatial spillovers.

\section{A.7 A trade model with intermediate goods and tariffs}

We now consider a Ricardian model based on the seminal work of Eaton and Kortum (2002) but augmented to include tariffs and an input-output network as in Alvarez and Lucas (2007). 


\section{A.7.1 The model}

\section{Setup}

There are $N$ locations, each of which produces 3 sets of goods: a continuum of tradeables $q_{i}(u)$ where $u \in[0,1]$, a aggregate intermediate good $a_{i}$, and a non-tradeable final good $c_{i}$. Agents in the economy derive their utility from the non-tradeable final good $c_{i}$. This final good $c_{i}$ is produced in a Cobb-Douglas manner using the intermediate good $a_{i}$ and labor i.e. $c_{i}=s_{f i}^{\alpha} a_{f i}^{1-\alpha}$ where $s_{f i}$ and $a_{f i}$ are the labor and intermediate inputs in final good production, respectively. The intermediate good $a_{i}$ is a Spence-Dixit-Stiglitz aggregate of all varieties of tradeables:

$$
a_{i}=\left[\int_{0}^{1}\left(q_{i^{*}}(u)\right)^{1-1 / \eta} d u\right]^{\frac{\eta}{\eta-1}}
$$

where $i^{*} \equiv \arg \min _{j \in \mathcal{N}} p_{j i}(u)$, i.e. each variety of tradeable is sourced from the lowest cost location. Tradeables in turn are produced using the composite intermediate good $I_{i}$ as input, along with labor as:

$$
q_{i}(u)=x_{i}(u)^{-\theta} s_{i}(u)^{\beta} a_{i}(u)^{1-\beta}
$$

where $x_{i}(u)^{-\theta}$ is the total factor productivity, $a_{i}(u)$ is the quantity of the intermediate good used in the production of tradeable variety $u$ and $s_{i}(u)$ is the labor input. Following Alvarez and Lucas (2007), we assume $x_{i}(u)$ follows an exponential distribution with parameter $\lambda_{i}$ and its draws are independent across $u$ (and across countries), allowing us to rewrite the above equations in terms of $x$. Each country $i \in\{1,2, \ldots N\} \equiv \mathcal{N}$ is endowed with immobile labor $L_{i}$. Transportation costs between countries are iceberg in nature, where to keep the notation similar to Alvarez and Lucas (2007), we denote by $\kappa_{i j} \leq 1$ as the fraction arriving in location $j \in \mathcal{N}$ if one unit is set from location $i \in \mathcal{N}$. Tariffs $\omega_{i j}$ are defined as the proportion of revenue received by producer in country

$j$ for a unit of its tradeable good sold in country $i$. In addition, we define $Y_{m i}$ as the revenue of the tradeables sector and $I_{i}$ as the expenditure on tradeables in country $i$.

\section{Equilibrium}

The equilibrium can be characterized by three sets of equations. The first one corresponds to equation 3.8 in Alvarez and Lucas (2007):

$$
p_{m i}^{-1 / \theta}=\sum_{j \in \mathcal{N}} \lambda_{j}\left(\frac{1}{\kappa_{i j}} \frac{A B}{\omega_{i j}}\right)^{-1 / \theta}\left(w_{j}^{\beta} p_{m j}^{1-\beta}\right)^{-1 / \theta} .
$$

Now we derive the other two, which are different from those in Alvarez and Lucas (2007) and convenient for the exercise here. Let $L_{f i}$ and $L_{m i}$ be the numbers of labor used in country $i$, production of the final and intermediate goods. We have $\alpha Y_{f i}=L_{f i} w_{i}$ and $\beta Y_{m i}=L_{m i} w_{i}$. Adding both sides of the two equations, we get

$$
\alpha Y_{f i}+\beta Y_{m i}=L_{i} w_{i}
$$

Also let $T_{i}$ be the total tariffs collected by country $i$. Notice that the residents' total income in country $i$ is $L_{i} w_{i}+T_{i}$ and all used to buy the final goods. That is $Y_{f i}=L_{i} w_{i}+T_{i}$. Substitute the expression into the above displayed equation. We can solve

$$
L_{i} w_{i}=\frac{\alpha}{1-\alpha} T_{i}+\frac{\beta}{1-\alpha} Y_{m i}
$$


Let $I_{j}$ be the total expenditure on intermediate goods in country $j$. Then $D_{j i} I_{j}$ is the amount spent on intermediate goods from country $i$, of which $\left(1-\omega_{j i}\right) D_{j i} I_{j}$ is tariff and goes to the government and $\omega_{j i} D_{j i} I_{j}$ goes to the producer. Thus, we have $T_{i}=\sum_{j \in \mathcal{N}}\left(1-\omega_{j i}\right) D_{j i} I_{j}$ and $Y_{m i}=\sum_{j \in \mathcal{N}} \omega_{j i} D_{j i} I_{j}$. Insert them into equation (32). Then we get our second equilibrium equation

$$
L_{i} w_{i}=\sum_{j \in \mathcal{N}} \frac{\alpha\left(1-\omega_{j i}\right)+\beta \omega_{j i}}{1-\alpha} D_{j i} I_{j}
$$

Furthermore, notice that producers' total expenditure $I_{i}+L_{i} w_{i}$ must be equal to their total income $Y_{m i}+Y_{f i}$ i.e. $I_{i}+L_{i} w_{i}=Y_{m i}+Y_{f i}$. Since $L_{i} w_{i}+T_{i}=Y_{f i}, I_{i}$ must be equal to $Y_{m i}+T_{i}$. Substituting the expression of $Y_{m i}$ and $T_{i}$, we then have

$$
I_{i}=\sum_{j \in \mathcal{N}} D_{j i} I_{j}
$$

Although the above equilibrium equations can be simply transformed the one in Theorem 1, unfortunately, the corresponding spectral radius we get is larger than $1 .{ }^{15}$ We move to impose a quasi-symmetry condition like Allen and Arkolakis (2014) that can allow us to reduce the three sets of equilibrium equations into two. Specifically, we assume $\kappa_{i j} \omega_{i j}=\tau_{i j} c_{i} r_{j}$ for some $\tau_{i j}, c_{i}$, and $r_{j}$ where $\tau$ is symmetric i.e. for any $i, j, \tau_{i j}=\tau_{j i}$.

Notice that $\sum_{j \in \mathcal{N}} D_{i j}=1$. Multiplying it with both sides of equation (34), we get $\sum_{j \in \mathcal{N}} D_{i j} I_{i}=$ $\sum_{j \in \mathcal{N}} D_{j i} I_{j}$. Substitute into the expression of equation $D_{i j}=\lambda_{j}\left(\frac{w_{j}^{\beta} p_{m j}^{1-\beta}}{p_{m i}}\right)^{-1 / \theta}\left(\frac{A B}{\kappa_{i j} \omega_{i j}}\right)^{-1 / \theta}$ and $\kappa_{i j} \omega_{i j}=\tau_{i j} c_{i} r_{j}$, then:

$$
\sum_{j \in \mathcal{N}} \lambda_{j}\left(\frac{w_{j}^{\beta} p_{m j}^{1-\beta}}{p_{m i}}\right)^{-1 / \theta}\left(\frac{A B}{\tau_{i j} c_{i} r_{j}}\right)^{-1 / \theta} I_{i}=\sum_{j \in \mathcal{N}} \lambda_{i}\left(\frac{w_{i}^{\beta} p_{m i}^{1-\beta}}{p_{m j}}\right)^{-1 / \theta}\left(\frac{A B}{\tau_{j i} c_{j} r_{i}}\right)^{-1 / \theta} I_{j}
$$

On the left side of this equation, keep all the $i$-related variables $\left(c_{i}, p_{m i}\right.$, and $\left.I_{i}\right)$ and move the rest (the summation) to the right side; similarly, on the right side of this equation, keep all the $i$-related variables $\left(\lambda_{i}, r_{i}, w_{i}\right.$, and $\left.p_{m i},\right)$ and move the rest (the summation) to the left side. We have:

$$
\frac{c_{i}^{1 / \theta} p_{m i}^{1 / \theta} I_{i}}{\sum_{j \in \mathcal{N}}\left(\frac{A B}{\tau_{j i}}\right)^{-1 / \theta} c_{j}^{1 / \theta} p_{m j}^{1 / \theta} I_{j}}=\frac{\lambda_{i} r_{i}^{1 / \theta}\left(w_{i}^{\beta} p_{m i}^{1-\beta}\right)^{-1 / \theta}}{\sum_{j \in \mathcal{N}}\left(\frac{A B}{\tau_{i j}}\right)^{-1 / \theta} \lambda_{j} r_{j}^{1 / \theta}\left(w_{j}^{\beta} p_{m j}^{1-\beta}\right)^{-1 / \theta}}
$$

Denote the numerators, $c_{i}^{1 / \theta} p_{m i}^{1 / \theta} I_{i}$ and $\lambda_{i} r_{i}^{1 / \theta}\left(w_{i}^{\beta} p_{m i}^{1-\beta}\right)^{-1 / \theta}$, as $\tilde{I}_{i}$ and $\tilde{w}_{i}$ respectively. Furthermore, denote $\left(\frac{A B}{\tau_{i j}}\right)^{-1 / \theta}$ as $\tilde{\tau}_{i j}$. Notice that $\tilde{\tau}_{i j}=\tilde{\tau}_{j i}$. Then we can write the above equation as $\frac{\tilde{I}_{i}}{\sum_{j \in \mathcal{N}} \tilde{\tau}_{i j} \tilde{I}_{j}}=\frac{\tilde{w}_{i}}{\sum_{j \in \mathcal{N}} \tilde{\tau}_{i j} \tilde{w}_{j}}$, of which the value we denote as $\gamma_{i}$. Then we can write this equation as

\footnotetext{
${ }^{15}$ This does not necessarily imply multiplicity of solutions since the kernels are correlated unlike Part (iii).
} 
two equations:

$$
\begin{aligned}
\tilde{I}_{i} & =\sum_{j \in \mathcal{N}} \gamma_{i} \tilde{\tau}_{i j} \tilde{I}_{j} \\
\tilde{w}_{i} & =\sum_{j \in \mathcal{N}} \gamma_{i} \tilde{\tau}_{i j} \tilde{w}_{j}
\end{aligned}
$$

Thus $\tilde{I}$ and $\tilde{w}$ can be viewed as two solutions of equation $x_{i}=\sum_{j \in \mathcal{N}} \gamma_{i} \tilde{\tau}_{i j} x_{j}$. According to Perron-Frobenius theorem, $\tilde{I}$ and $\tilde{w}$ is different at most up to scale i.e. there exists some constant $a$ such that $\tilde{I}_{i}=a \tilde{w}_{i}$. Furthermore, substitute into the expression of $\tilde{I}_{i}$ and $\tilde{w}_{i}$ and we get

$$
I_{i}=a \lambda_{i} r_{i}^{1 / \theta} c_{i}^{-1 / \theta}\left(w_{i}^{\beta} p_{m i}^{2-\beta}\right)^{-1 / \theta}
$$

Notice that in this expression, $I_{i}, w_{i}$, and $p_{m i}$ are nominal variables and we can scale them arbitrarily and get the corresponding $a$. Therefore, $a$ simply reflects the normalization of nominal variables and without loss of generality, we set $a=1$.

Substitute equation (35) into equation (33). Then, the equilibrium can be characterized by equations (31) and (33) where the endogenous variables are: $p_{m i}$, the price index of tradeables in country $i$; and $w_{i}$, country $i$ 's wage.

\section{Applying Theorem 1}

As in the previous example, the equilibrium of this system can be expressed in the special form of equation (1) presented in equation (3) in Remark 5 . Now we show how to transform the equilibrium equations into the form of equation (3). To see this, denote $\lambda_{j}\left(\frac{1}{\kappa_{i j}} \frac{A B}{\omega_{i j}}\right)^{-1 / \theta}$ in equation (31) as $K_{i j}^{1}$, so that it becomes

$$
p_{m i}^{-1 / \theta}=\sum_{j \in \mathcal{N}} K_{i j}^{1}\left(w_{j}^{\beta} p_{m j}^{1-\beta}\right)^{-1 / \theta}
$$

Second, substitute the expression of $D_{i j}=\lambda_{j}\left(\frac{w_{j}^{\beta} p_{m j}^{1-\beta}}{p_{m i}}\right)^{-1 / \theta}\left(\frac{A B}{\kappa_{i j} \omega_{i j}}\right)^{-1 / \theta}$ and $I_{i}=a \lambda_{i} r_{i}^{1 / \theta} c_{i}^{-1 / \theta}\left(w_{i}^{\beta} p_{m i}^{2-\beta}\right)^{-1 / \theta}$ into equation (33), multiply both sides by $\left(w_{i}^{\beta} p_{m i}^{1-\beta}\right)^{1 / \theta} L_{i}^{-1}$, and denote $\frac{\alpha\left(1-\omega_{j i}\right)+\beta \omega_{j i}}{1-\alpha}\left(\frac{A B}{\kappa_{j i} \omega_{j i}}\right)^{-1 / \theta} \lambda_{i} \lambda_{j} r_{j}^{1 / \theta} c_{j}^{-1 / \theta} L_{i}^{-1}$ as $K_{i j}^{2}$, so that equation (33) becomes

$$
w_{i}^{1+\beta / \theta} p_{m i}^{(1-\beta) / \theta}=\sum_{j \in \mathcal{N}} K_{i j}^{2}\left(w_{j}^{\beta} p_{m j}^{1-\beta}\right)^{-1 / \theta} .
$$

Now we have transformed the equilibrium equations into the form (3) and with two set of endogenous variables $\left\{p_{m i}, w_{i}\right\}_{i=1,2, \ldots, n}$. Notice that the kernels, $K_{i j}^{1}$ and $K_{i j}^{2}$, defined above are positive when $\alpha, \beta, \theta>0$ and $0<\omega_{i j} \leq 1$.

Then we have the corresponding parameter matrices

$$
\Gamma=\left(\begin{array}{cc}
-1 / \theta & 0 \\
(1-\beta) / \theta & 1+\beta / \theta
\end{array}\right), \mathbf{B}=\left(\begin{array}{cc}
-(1-\beta) / \theta & -\beta / \theta \\
-(1-\beta) / \theta & -\beta / \theta
\end{array}\right)
$$

Clearly, $\Gamma$ is always invertible as long as $\theta>0$. Therefore, we have 


$$
\left|\mathbf{B} \boldsymbol{\Gamma}^{-\mathbf{1}}\right|=\left(\begin{array}{cc}
\frac{(1-\beta) \theta}{\beta+\theta} & \frac{\beta}{\beta+\theta} \\
\frac{(1-\beta) \theta}{\beta+\theta} & \frac{\beta}{\beta+\theta}
\end{array}\right)
$$

Here $\rho\left(\left|\mathbf{B} \boldsymbol{\Gamma}^{-1}\right|\right)=\frac{\beta+\theta-\beta \theta}{\beta+\theta}<1$ i.e. we always have (up-to-scale) uniqueness with quasi-symmetry trade costs $\kappa_{i j}$ and tariffs $\omega_{i j}$. In comparison, the conditions for uniqueness in Alvarez and Lucas (2007) (see their Theorem 3) are:

$$
\text { (i) }\left(\min _{i, j \in \mathcal{N}^{2}}\left\{\kappa_{i j}\right\} \min _{i, j \in \mathcal{N}^{2}}\left\{\omega_{i j}\right\}\right)^{\frac{2}{\theta}} \geq 1-\beta \text {; (ii) } \alpha \geq \beta \text {; (iii) } 1-\min _{i, j \in \mathcal{N}^{2}}\left\{\omega_{i j}\right\} \leq \frac{\theta}{\alpha-\beta},
$$

although these conditions are derived only for the special case of uniform tariffs (i.e. $\omega_{i j}=\omega_{i}$ for all $j \in \mathcal{N})$.

\section{A.8 A production network with multiple intermediates goods}

We extend the many firm production network in the seminal paper by Acemoglu, Carvalho, Ozdaglar, and Tahbaz-Salehi (2012) to include (1) a constant elasticity of substitution (CES) aggregator across labor and intermediates (as discussed in Carvalho and Tahbaz-Salehi (2019)), (2) a constant elasticity of substitution between intermediate goods (as discussed in Carvalho, Nirei, Saito, and Tahbaz-Salehi (2021)), and (3) multiple types of intermediates goods.

\section{A.8.1 The model}

Setup There are $N$ different competitive firms, each of which produce $H$ distinct products using as intermediate goods the output of all other firms. The quantity of product $H$ by firm $i \in\{1, \ldots, N\} \equiv$ $\mathcal{N}, Q_{i h}$, is determined by a constant elasticity of substitution production function combining labor and a composite bundle of intermediate goods as follows:

$$
Q_{i h}=\left[\left(1-\mu_{i h}\right)^{\frac{1}{\zeta_{h}}}\left(A_{i h} L_{i h}\right)^{\frac{\zeta_{h}-1}{\zeta_{h}}}+\mu_{i h}^{\frac{1}{\zeta_{h}}} M_{i h}^{\frac{\zeta_{h}-1}{\zeta_{h}}}\right]^{\frac{\zeta_{h}}{\zeta_{h}-1}}
$$

where $\mu_{i h}$ is between 0 and 1 and governs the relative importance of labor and intermediate goods, $L_{i h}$ is the amount of labor, $A_{i h}$ is the (exogenous) labor productivity, $\zeta_{h}$ is the elasticity of substitution between labor and intermediates, and the intermediate input bundle $M_{i h}$ is a CES aggregate of inputs purchased from other firms:

$$
M_{i h}=\prod_{h^{\prime} \in \mathcal{H}}\left(\left(\sum_{j \in \mathcal{N}} a_{j i h^{\prime} h}^{\frac{1}{\sigma_{h^{\prime}}}} q_{j i h^{\prime} h}^{\frac{\sigma h^{\prime}-1}{\sigma h^{\prime}}}\right)^{\frac{\sigma_{h^{\prime}}}{\sigma_{h^{\prime}}-1}}\right)^{\beta_{h^{\prime} h}},
$$

where $\sigma_{h^{\prime}}$ is the elasticity of substitution between different intermediate goods, $a_{j i h^{\prime} h}$ represents the production network of firms $j$ supplying intermediates $h^{\prime}$ in firm $i$ 's production of product $h$, $q_{j i h^{\prime} h}$ is the quantity supplied, and $\left\{\beta_{h^{\prime} h}\right\}_{h^{\prime} \in \mathcal{H}}$ is the intermediates share satisfying $\sum_{h^{\prime}} \beta_{h^{\prime} h}=1$ for all $h$. 
Equilibrium Solving the cost minimization problem of the firm results in the following system of equations for equilibrium firm prices $p_{i h}$ :

$$
p_{i h}^{1-\zeta_{h}}=\left(1-\mu_{i h}\right)\left(w / A_{i h}\right)^{1-\zeta_{h}}+\mu_{i h} \prod_{h^{\prime} \in \mathcal{H}} \beta_{h^{\prime} h}^{-\beta_{h^{\prime} h}}\left(\sum_{j=1}^{n} a_{j i h^{\prime} h} p_{j h^{\prime}}^{1-\sigma_{h^{\prime}}}\right)^{\frac{\beta_{h^{\prime} h}}{1-\sigma_{h^{\prime}}}\left(1-\zeta_{h}\right)}
$$

where $w$ is the (exogenous) market wage.

\section{A.8.2 Applying Theorem 1}

Take both sides of equation (38) to the power of $\frac{1}{1-\zeta_{h}}$ and denote its right side as $F_{i h}(\cdot)$. We can directly bound its elasticity as follows:

$$
\sum_{j \in \mathcal{N}}\left|\frac{\partial \ln F_{i h}}{\partial \ln p_{j h^{\prime}}}\right|<\beta_{h^{\prime} h}
$$

Since $\sum_{h^{\prime}} \beta_{h^{\prime} h}=1$, according to Remark 1, we have $\rho(\beta)=1$. Thus by Theorem 1 (part ii.a) and Remark 1, there exists at most one equilibrium. To our knowledge, this is the first proof of uniqueness of an equilibrium in a many firm production network with multiple types of intermediates goods and constant elasticity of substitution between different types of intermediate goods and between the intermediate goods bundle and labor.

\section{A.9 Productivity identification in a production network with many loca- tions and sectors}

In this application, we consider input-output production networks with many locations and sectors as in the seminal paper of Caliendo and Parro (2015). The purpose of this is two-fold: first, it demonstrates how Theorem 1 can be applied to establish identification results (in addition to characterizing the uniqueness of the equilibrium, as in the examples in the main text); second, it demonstrates the ubiquity of economic situations where $\rho(\mathbf{A})=1$ (see Remark 6), highlighting the importance of part (ii) of Theorem 1.

\section{A.9.1 The Model}

Setup Consider an economy comprised of $i \in\{1, \ldots, N\} \equiv \mathcal{N}$ locations and $h \in\{1, \ldots, H\} \equiv$ $\mathcal{H}$ sectors. Each sector $h$ in location $i$ produces a differentiated intermediate good (denoted as good $(i, h)$ ) by combining local labor with a Cobb-Douglas combination of a CES composite of intermediates from all locations according to the following production function:

$$
Q_{i h}=A_{i h} L_{i h}^{\alpha_{h}} \prod_{h^{\prime} \in \mathcal{H}}\left(\left(\sum_{j \in \mathcal{N}} \frac{\sigma_{h^{\prime}-1}}{q_{h^{\prime} h}}\right)^{\frac{\sigma_{h^{\prime}}}{\sigma_{h^{\prime}}-1}}\right)^{\beta_{h^{\prime} h}},
$$

where $q_{j i h^{\prime} h}$ is the quantity of the good $\left(j, h^{\prime}\right)$ used as an intermediate good in the production of $\operatorname{good}(i, h),\left\{\sigma_{h}\right\}_{h \in \mathcal{H}}$ are the sector elasticities of substitution across locations, $\left\{\alpha_{h}\right\}_{h \in \mathcal{H}}$ are the sector labor shares, and $\mathbf{B} \equiv\left[\beta_{h^{\prime} h}\right]$ is an $H \times H$ input-output matrix of intermediate inputs, and 
$\left\{A_{i h}\right\}_{i \in \mathcal{N}}^{h \in \mathcal{H}}$ are the productivities of each sector-location. The shipment of $\operatorname{good}\left(j, h^{\prime}\right)$ from $j \in \mathcal{N}$ to $i \in \mathcal{N}$ incurs an iceberg trade cost $\tau_{i j h^{\prime}} \geq 1$.

Suppose that each location $i \in \mathcal{N}$ is endowed with $L_{i}$ agents, each of whom is perfectly mobile across sectors and earns (equilibrium) wage $w_{i}$ for inelastically supplying one unit of labor. Agents use their wages to consume a non-traded final good produced by combining intermediate goods with the production function $C_{i}=\prod_{\substack{h \in \mathcal{H} \\ \sigma_{h}}} M_{i h}^{\gamma_{h}}$, where $\sum_{h \in \mathcal{H}} \gamma_{h}=1$ are the consumption shares of each sector, $M_{i h}=\left(\sum_{j \in \mathcal{N}} m_{j h}^{\frac{\sigma_{h}-1}{\sigma_{h}}}\right)^{\frac{\sigma_{h}}{\sigma_{h}-1}}$, and $m_{j h}$ is the quantity of the good $(j, h)$ used in the production of final good.

Equilibrium From the cost minimization, the equilibrium price of the intermediate good produced by sector $h \in \mathcal{H}$ in location $i \in \mathcal{N}$ is:

$$
p_{i h}=c_{h} \frac{1}{A_{i h}} w_{i}^{\alpha_{h}} \prod_{h^{\prime} \in \mathcal{H}} P_{i h^{\prime}}^{\beta_{h^{\prime} h}}
$$

where

$$
P_{i h}^{1-\sigma_{h}}=\sum_{j \in \mathcal{N}} \tau_{j i h}^{1-\sigma_{h}} p_{j h}^{1-\sigma_{h}}
$$

is a sector-location price index of intermediate goods purchased in all locations, and $c_{h}>0$ is a exogenous constant. ${ }^{16}$

Let $Y_{i h} \equiv p_{i h} Q_{i h}$ denote the income of sector $h \in \mathcal{H}$ in location $i \in \mathcal{N}$, which in equilibrium is equal to its total sales to all locations and sectors:

$$
Y_{i h}=\sum_{j \in \mathcal{N}} \tau_{i j h}^{1-\sigma_{h}} p_{i h}^{1-\sigma_{h}} P_{j h}^{\sigma_{h}-1} \sum_{h^{\prime} \in \mathcal{H}}\left(\beta_{h h^{\prime}}+\gamma_{h} \alpha_{h^{\prime}}\right) Y_{j h^{\prime}}
$$

where the two terms in the last summation captures how much spending in sector $\left(j, h^{\prime}\right)$ translates to spending in sector $(i, h)$ through intermediate production and final good purchases by consumers, respectively.

Identification The question we are interested in is the following. Suppose one observes (1) the sales of each sector $h \in \mathcal{H}$ in each location $i \in \mathcal{N}$, i.e. $\left\{Y_{i h}\right\}_{i \in \mathcal{N}}^{h \in \mathcal{H}} ;(2)$ the labor endowment $\left\{L_{i}\right\}_{i \in \mathcal{N}}$; (3) the sector elasticities $\left\{\sigma_{h}\right\}_{h \in \mathcal{H}}$; (4) the sector production function labor shares $\left\{\alpha_{h}\right\}_{h \in \mathcal{H}}$ and input-output matrix $\mathbf{B} \equiv\left[\beta_{h^{\prime} h}\right]$; (5) the final good production shares $\left\{\gamma_{h}\right\}_{h \in \mathcal{H}}$; and (6) the sectorspecific bilateral trade costs $\left\{\tau_{i j h}\right\}_{i, j \in \mathcal{N}}^{h \in \mathcal{H}}$. Is it possible to identify the productivity of each sector $h \in \mathcal{H}$ in each location $i \in \mathcal{H},\left\{A_{i h}\right\}_{i \in \mathcal{N}}^{h \in \mathcal{H}}$ ? One could imagine many instances where recovering the underlying productivities of different sectors in different locations from observed sales data is useful and important: e.g. in the study of comparative advantage, structural change, technological innovations, etc.

\section{A.9.2 Applying Theorem 1}

We begin by remarking that since wages can be inferred directly from the observed labor share of income and labor endowment, given knowledge of prices $\left\{p_{i h}\right\}_{i \in \mathcal{N}}^{h \in \mathcal{H}}$ and price indices $\left\{P_{i h}\right\}_{i \in \mathcal{N}}^{h \in \mathcal{H}}$,

\footnotetext{
${ }^{16}$ In particular, $c_{h} \equiv \alpha_{h}^{-\alpha_{h}} \prod_{h^{\prime} \in \mathcal{H}} \beta_{h^{\prime} h}^{-\beta_{h^{\prime} h}}$.
} 
one can immediately recover productivities $\left\{A_{i h}\right\}_{i \in \mathcal{N}}^{h \in \mathcal{H}}$ from equation (39). Hence, it is sufficient to focus on the question of identification of prices $\left\{p_{i h}\right\}_{i \in \mathcal{N}}^{h \in \mathcal{H}}$ and price indices $\left\{P_{i h}\right\}_{i \in \mathcal{N}}^{h \in \mathcal{H}}$.

Define the $2 H \times 1$ endogenous vector $\boldsymbol{x}_{i}=\left[\left\{P_{i h}^{1-\sigma_{h}}\right\}_{h=1}^{H},\left\{p_{i h}^{\sigma_{h}-1}\right\}_{h=1}^{H}\right]$ so that equations (40) and (41) can be written as:

$$
x_{i h}= \begin{cases}\sum_{j} K_{i j, h} x_{j, h+H}^{-1} & \text { if } h \in\{1, \ldots, H\} \\ \sum_{j} F_{i j, h} x_{j, h-H}^{-1} & \text { if } h \in\{H+1, \ldots, 2 H\}\end{cases}
$$

where $K_{i j, h} \equiv \tau_{j i h}^{1-\sigma_{h}}$ for $h \in\{1, \ldots, H\}$ and $F_{i j, h} \equiv \tau_{i j h}^{1-\sigma_{h}}\left(\frac{\sum_{h^{\prime} \in \mathcal{H}}\left(\beta_{h h^{\prime}}+\gamma_{h} \alpha_{h^{\prime}}\right) Y_{j h^{\prime}}}{Y_{i h}}\right)$ for $h \in\{H+1, \ldots, 2 H\}$. As a result, we can define the $2 H \times 2 H$ matrix of elasticity bounds as:

$$
\mathbf{A} \equiv\left(\begin{array}{cc}
\mathbf{0} & \mathbf{I}_{H} \\
\mathbf{I}_{H} & \mathbf{0}
\end{array}\right)
$$

where $\mathbf{I}_{H}$ is the $H \times H$ identity matrix. Hence, regardless of the particular input output structure (or the values of labor shares, final goods shares, or sector elasticities) we have $\rho(\mathbf{A})=1$, and so from Theorem 1 part (ii) there is at most one set of (column-wise to scale unique) prices $\left\{p_{i h}\right\}_{i \in \mathcal{N}}^{h \in \mathcal{N}}$ and price indices $\left\{P_{i h}\right\}_{i \in \mathcal{N}}^{h \in \mathcal{H}}$ consistent with equations (40) and (41). This then implies that there is at most one (column-wise up to scale) unique set of productivities $\left\{A_{i h}\right\}$ consistent with observed sales data. ${ }^{17}$ To our knowledge, this is the first identification result applied to many location/sector models with input/output linkages.

\section{A.10 Inverting a demand system with multiple types of goods}

Here we consider the question of the invertibility of demand systems based on the seminal work of Berry, Levinsohn, and Pakes (1995). In Berry, Levinsohn, and Pakes (1995), agents makes a choice over a single type of goods, e.g. which cellphone to buy. Here, we extend the framework to consider a situation where consumers simultaneously make decisions across multiple types of goods, e.g. which cellphone and computer to buy. We suppose that the market shares for each type are observed and ask if that is enough information to recover the unobserved demand for each good. ${ }^{18}$

\section{A.10.1 The model}

Setup There are $H$ types of goods for agents to buy (e.g. cellphones, computers, and automobiles). Within each type $h \in\{1, \ldots, H\} \equiv \mathcal{H}$ of good, there are $N_{h}$ products over which to choose (e.g. in the case of cellphones, there are the Google Pixel 6, the iPhone 13, etc.). One of these $N_{h}$ products may be the choice to purchase nothing.

Let $J$ be a $H$-by- 1 vector representing agent's choice over the bundle of products. Specifically, $J \equiv\left[j_{h}\right]_{h \in \mathcal{H}}$, where $j_{h} \in\left\{1, \ldots, N_{h}\right\} \equiv \mathcal{N}_{h}$ is agent's choice of product type $h$ to purchase. Suppose that the latent utility of agent $k$ 's choice $J$ is:

\footnotetext{
${ }^{17}$ The column-wise up to scale uniqueness implies that the relative productivity within sector across locations can be identified from sales data, but the relative productivity across sectors cannot; intuitively, if the productivity of sector $h$ doubles in all locations, given the unit price elasticity from the presumed Cobb-Douglas production function, its price will half, leaving its sales unchanged.

${ }^{18}$ While the choice of buying two products can be technically modeled as a single choice over pairs of products, applying the inversion results of Berry, Levinsohn, and Pakes (1995) would then require knowledge of the market shares of each pair of products, which is typically not observed.
} 


$$
U_{k}(J)=\sum_{h \in \mathcal{H}} \delta_{j_{h}, h}+\mu\left(J, \nu_{k}\right)+\varepsilon_{k J}
$$

where $\delta_{j_{h}, h}$ represents the (unobservable) good characteristics of product $j_{h}$ in type $h, \mu\left(J, \nu_{k}\right)$ is a function of (observable) good characteristics of the bundle $J$ and consumer characteristics $\nu_{k}$ and $\varepsilon_{k J}$ is a random variable representing agents' idiosyncratic preference. Note that $\mu\left(J, \nu_{k}\right)$ flexibly allows for any sort of (observed) complementarity or substitutability across products of different types, which can potentially vary with consumer characteristics $\nu_{k}$. We assume $\nu_{k} \sim P$ where $P$ is a known distribution and $\varepsilon_{k J}$ has type I extreme value distributions independent of $k$ and $J$.

Suppose for each $h \in \mathcal{H}$ we observe the fraction of agents that choose product $i \in \mathcal{N}_{h}$, i.e. the market share $s_{i, h}$. Our goal is to identify the set of unobservable good characteristrics $\left\{\delta_{i, h}\right\}$.

Market share Given the extreme value distribution of $\varepsilon_{i h}$, the market share can be written as:

$s_{i, h}=\int \frac{\exp \left(\delta_{i, h}\right) \sum_{j_{1}=1}^{N_{1}} \cdots \sum_{j_{h-1}=1}^{N_{h-1}} \sum_{j_{h+1}=1}^{N_{h+1}} \cdots \sum_{j_{H}=1}^{N_{H}} \exp \left(\sum_{h^{\prime} \neq h} \delta_{j_{h^{\prime}}, h^{\prime}}+\mu\left(\left[j_{1}, \ldots, j_{h-1}, i, j_{h+1}, \ldots, j_{H}\right], \nu\right)\right)}{\sum_{j_{h}=1}^{N_{h}} \exp \left(\delta_{j_{h}, h}\right) \sum_{j_{1}=1}^{N_{1}} \cdots \sum_{j_{h-1}=1}^{N_{h}} \sum_{j_{h+1}=1}^{N_{h+1}} \cdots \sum_{j_{H}=1}^{N_{H}} \exp \left(\sum_{h^{\prime} \neq h} \delta_{j_{h^{\prime}}, h^{\prime}}+\mu\left(\left[j_{1}, \ldots, j_{h-1}, j_{h}, j_{h+1}, \ldots, j_{H}\right], \nu\right)\right)} d P(\nu)$.

\section{A.10.2 Applying Theorem 1}

The case of $H=1$ (Berry, Levinsohn, and Pakes (1995)) We first consider the case of $H=1$, as in Berry, Levinsohn, and Pakes (1995). In this case, equation (43) becomes:

$$
s_{i}=\int \frac{\exp \left(\delta_{i}+\mu(i, \nu)\right)}{\sum_{j=1}^{N} \exp \left(\delta_{j}+\mu(j, \nu)\right)} d P(\nu) .
$$

Define $x_{i} \equiv \exp \left(\delta_{i}\right)$. Then $x_{i}=s_{i} f_{i}(x)$,where $f_{i}(x) \equiv\left(\int \frac{\exp (\mu(i, \nu))}{\sum_{j=1}^{N} x_{j} \exp (\mu(j, \nu))} d P(\nu)\right)^{-1}$. We then have:

$$
\frac{\partial \ln f_{i}}{\partial \ln x_{j}}=f_{i} \int \frac{\exp (\mu(i, \nu)) x_{j} \exp (\mu(j, \nu))}{\left(\sum_{j=1}^{N} x_{j} \exp (\mu(j, \nu))\right)^{2}} d P \nu
$$

which in turn implies:

$$
\begin{aligned}
\sum_{j \in \mathcal{N}}\left|\frac{\partial \ln f_{i}}{\partial \ln x_{j}}\right| & =f_{i} \int \frac{\exp (\mu(i, \nu)) \sum_{j} x_{j} \exp (\mu(j, \nu))}{\left(\sum_{j=1}^{N} x_{j} \exp (\mu(j, \nu))\right)^{2}} d P \nu \\
& =f_{i}(x) / f_{i}(x)=1 .
\end{aligned}
$$

According to part (ii) of Theorem 1 and Remark 1, there is at most one set of $\left\{\delta_{i}\right\}$ (up to an unknown constant), as in Berry, Levinsohn, and Pakes (1995).

The case of $H=2$ We now consider the case of $H=2$, under the special case where $\mu([i, j], \nu) \equiv$ $\mu_{p}([i, j])+\mu_{c}(\nu)$, i.e. that there is separability between any complementarity or substitutability of product characteristics and any heterogeneity in consumer preferences. Also, we assume $N_{1}=$ $N_{2}=N$. 
Define $x_{i, h} \equiv \exp \left(\delta_{i, h}\right)$. Equation (43) can be written as:

$$
\begin{aligned}
x_{i, 1} z_{i, 2} & =\sum_{j=1}^{N} s_{i, 1} x_{j, 1} z_{j, 2} \\
x_{i, 2} z_{i, 1} & =\sum_{j=1}^{N} s_{i, 2} x_{j, 2} z_{j, 1},
\end{aligned}
$$

where:

$$
\begin{aligned}
z_{i, 1} & \equiv \sum_{j=1}^{N} x_{j, 1} \exp \left(\mu_{p}([j, i])\right) \\
z_{i, 2} & \equiv \sum_{j=1}^{N} x_{j, 2} \exp \left(\mu_{p}([i, j])\right)
\end{aligned}
$$

It is immediately evident that this system of $4 N$ equations in $4 N$ unknowns takes the form of equation (3), which is a special case of equation (1), so by applying Remark 5 , it is sufficient to characterize the spectral radius of matrix $\mathbf{A} \equiv\left|\mathbf{B} \boldsymbol{\Gamma}^{-1}\right|$, where:

$$
\mathbf{B} \equiv\left(\begin{array}{llll}
1 & 0 & 0 & 1 \\
0 & 1 & 1 & 0 \\
1 & 0 & 0 & 0 \\
0 & 1 & 0 & 0
\end{array}\right), \boldsymbol{\Gamma} \equiv\left(\begin{array}{llll}
1 & 0 & 0 & 1 \\
0 & 1 & 1 & 0 \\
0 & 0 & 1 & 0 \\
0 & 0 & 0 & 1
\end{array}\right)
$$

so that:

$$
\mathbf{A} \equiv\left(\begin{array}{llll}
1 & 0 & 0 & 0 \\
0 & 1 & 0 & 0 \\
1 & 0 & 0 & 1 \\
0 & 1 & 1 & 0
\end{array}\right)
$$

which has a spectral radius equal to 1 , so that from Theorem 1 part (ii) there exists at most one set of $\left\{\delta_{i, h}\right\}$ consistent with the observed market shares, up to an unknown constant for each $h \in \mathcal{H}$, thereby extending the results of Berry, Levinsohn, and Pakes (1995) to the case of $H=2$ under the special case where $\mu([i, j], \nu) \equiv \mu_{p}([i, j])+\mu_{c}(\nu)$.

\section{B Additional Details}

\section{B.1 Details of Remarks}

In this section, we provide further details for the remarks discussed in the paper.

\section{B.1.1 Remark 1}

Remark 1: Suppose there exists an $H$-by- $H$ matrix A such that for all $i, j \in \mathcal{N}, h, h^{\prime} \in \mathcal{H}$, and $x_{j} \in \mathbb{R}_{++}^{H}, \sum_{j \in \mathcal{N}}\left|\frac{\partial \ln \sum_{k \in \mathcal{N}} f_{i k h}(x)}{\partial \ln x_{j h^{\prime}}}\right| \leq(\mathbf{A})_{h h^{\prime}}$. Then:

(i). If $\rho(\mathbf{A})<1$, there exists a unique solution to equation (1) which can be computed by iteratively applying equation (1) with a rate of convergence $\rho(\mathbf{A})$; 
(ii). If $\rho(\mathbf{A})=1$ and:

a. For all $i \in \mathcal{N}$ and $h, h^{\prime} \in \mathcal{H}$ when $(\mathbf{A})_{h h^{\prime}} \neq 0$ there exists some $j$ such that for all $x_{j} \in \mathbb{R}_{++}^{H}$, $\sum_{j \in \mathcal{N}}\left|\frac{\partial \ln \sum_{k \in \mathcal{N}} f_{i k h}(x)}{\partial \ln x_{j h^{\prime}}}\right|<(\mathbf{A})_{h h^{\prime}}$, then equation (1) has at most one solution;

b. For all $x_{j}, \sum_{j \in \mathcal{N}} \frac{\partial \ln \sum_{k \in \mathcal{N}} f_{i k h}(x)}{\partial \ln x_{j h^{\prime}}}=\alpha_{h h^{\prime}} \in \mathbb{R}$ where $\left|\alpha_{h h^{\prime}}\right|=(\mathbf{A})_{h h^{\prime}}$ then there is at most one column-wise up-to-scale solution, i.e. for any $h \in \mathcal{H}$ and two solutions $x$ and $x^{\prime}$ it must be $x_{. h}^{\prime}=c_{h} x_{. h}$ for some scalar $c_{h}>0$.

Proving this remark requires only a small change to the proof of Theorem 1. Equality (11) becomes $\frac{\partial g_{i h}}{\partial y_{j h^{\prime}}}=\frac{\partial \ln \sum_{k} f_{i k h}(x)}{\partial \ln x_{j h^{\prime}}}$.

Correspondingly, in part (i), inequality (13) becomes

$$
\begin{aligned}
\left|g_{i h}(y)-g_{i h}\left(y^{\prime}\right)\right| & =\left|\sum_{h^{\prime} \in \mathcal{H}} \sum_{j \in \mathcal{N}} \frac{\partial g_{i h}(\hat{y})}{\partial y_{j h^{\prime}}}\left(y_{j h^{\prime}}-y_{j h^{\prime}}^{\prime}\right)\right| \\
& \leq \sum_{h^{\prime} \in \mathcal{H}} \sum_{j \in \mathcal{N}}\left|\frac{\partial \ln \sum_{k} f_{i k h}(\hat{x})}{\partial \ln x_{j h^{\prime}}}\right| \max _{j \in \mathcal{N}}\left|y_{j h^{\prime}}-y_{j h^{\prime}}^{\prime}\right| \\
& \leq \sum_{h^{\prime} \in \mathcal{H}}(\mathbf{A})_{h h^{\prime}} \max _{j \in \mathcal{N}}\left|y_{j h^{\prime}}-y_{j h^{\prime}}^{\prime}\right| .
\end{aligned}
$$

And in part (ii.b), equation (15) becomes

$$
y_{i h}-y_{i h}^{\prime}+\hat{s}_{h}=\sum_{h^{\prime} \in \mathcal{H}} \sum_{j \in \mathcal{N}} \frac{\partial \ln \sum_{k} f_{i k h}(x)}{\partial \ln x_{j h^{\prime}}}\left(y_{j h^{\prime}}-y_{j h^{\prime}}^{\prime}+s_{h^{\prime}}\right)
$$

The rest of the proof of Theorem 1 remains unchanged.

\section{B.1.2 Remark 2}

Remark 2: Consider the special case of equation (1) where $f_{i j h}: \mathbb{R}_{+}^{H} \rightarrow \mathbb{R}_{+} \equiv K_{i j h} g_{i j h}\left(x_{j 1}, \ldots, x_{j H}\right)$, where $K_{i j h} \geq 0$ and $g_{i j h}\left(x_{j}\right)$ is continuous, differentiable, and $g_{i j h}\left(x_{j}\right)>0$ for all $x_{j}>0$ so that equation (1) becomes $x_{i h}=\sum_{j=1}^{N} K_{i j h} g_{i j h}\left(x_{j 1}, \ldots, x_{j H}\right)$. Then if $\rho(\mathbf{A})<1$ and matrices $\left(K_{i j h}\right)_{i, j \in \mathcal{N}}$ for all $h$ are irreducible, there exists only one strictly positive solution. Weakly positive solutions, where for some $i, h, x_{i, h}^{*}=0$, may exist but will be asymptotically unstable,

The condition that matrices $\left(K_{i j h}\right)_{i, j \in \mathcal{N}}$ for all $h$ are irreducible implies that for any strictly positive $x, \sum_{j=1}^{N} K_{i j h} g_{i j h}\left(x_{j 1}, \ldots, x_{j H}\right)>0$. Thus we can apply Remark 1 to obtain the existence, uniqueness, and convergence of the solution. Observe that the convergence simply implies the unstableness of other weakly positive solutions.

\section{B.1.3 Remark 3}

Remark 3: Suppose for all $i, j \in \mathcal{N}, h, h^{\prime} \in \mathcal{H}, F_{i h}(x)$ has right and left partial derivatives with respect to $x_{j h^{\prime}}$ and denote they as $\frac{\partial_{+} F_{i h}(x)}{\partial x_{j h^{\prime}}}$ and $\frac{\partial_{-} F_{i h}(x)}{\partial x_{j h^{\prime}}}$. Equation $x_{i h}=F_{i h}(x)$ has a unique solution if (1) there exists an $H$-by- $H$ matrix A satisfying $\rho(\mathbf{A})<1$ such that for all $i \in \mathcal{N}, h, h^{\prime} \in$ $\mathcal{H}, \sum_{j} \max \left(\left|\frac{\partial_{+} F_{i h}(x)}{\partial x_{j h^{\prime}}}\right|,\left|\frac{\partial_{-} F_{i h}(x)}{\partial x_{j h^{\prime}}}\right|\right) \leq(\mathbf{A})_{h h^{\prime}}$ or (2) there exists an $N$-by- $N$ matrix $\mathbf{B}$ satisfying $\rho(\mathbf{B})<1$ such that for all $i, j \in \mathcal{N}, h \in \mathcal{H}, \sum_{h^{\prime}} \max \left(\left|\frac{\partial_{+} F_{i h}(x)}{\partial x_{j h^{\prime}}}\right|,\left|\frac{\partial_{-} F_{i h}(x)}{\partial x_{j h^{\prime}}}\right|\right) \leq(\mathbf{B})_{i j}$. Due to symmetry, we only need to prove the first condition. 
Given any $x$ and $x^{\prime}$, let $m_{i h}\left(t_{i h}\right) \equiv F_{i h}\left(\left(1-t_{i h}\right) x+t_{i h} x^{\prime}\right)$ be a function of $t_{i h}$ on interval $[0,1]$. Since each $F_{i h}(x)$ is left and right differentiable, so is $m_{i h}\left(t_{i h}\right)$. Suppose $m_{i h+}^{\prime}\left(t_{i h}\right)$ and $m_{i h-}^{\prime}\left(t_{i h}\right)$ are function $m_{i h}(\cdot)$ 's right and left derivatives at $t_{i h}$. According to a generalized mean value theorem, there exists some $t_{i h} \in(0,1)$ such that $\frac{m_{i h}(1)-m_{i h}(0)}{1-0}$ is between $m_{i h+}^{\prime}\left(t_{i h}\right)$ and $m_{i h-}^{\prime}\left(t_{i h}\right)$. Observe that $m_{i h+}^{\prime}\left(t_{i h}\right)=\sum_{j, h^{\prime}}\left[\frac{\partial_{+} F_{i h}(\hat{x})}{\partial x_{j h^{\prime}}} \mathbb{1}_{\Delta x_{j h^{\prime}}>0}+\frac{\partial_{-} F_{i h}(\hat{x})}{\partial x_{j h^{\prime}}} \mathbb{1}_{\Delta x_{j h^{\prime}}<0}\right] \Delta x_{j h^{\prime}}$ where $\hat{x} \equiv\left(1-t_{i h}\right) x+$ $t_{i h} x^{\prime}$ and $\Delta x_{j h^{\prime}} \equiv x_{j h^{\prime}}^{\prime}-x_{j h^{\prime}}$. Similarly, $m_{i h-}^{\prime}\left(t_{i h}\right)=\sum_{j, h^{\prime}}\left[\frac{\partial_{+} F_{i h}(\hat{x})}{\partial x_{j h^{\prime}}} \mathbb{1}_{\Delta x_{j h^{\prime}}<0}+\frac{\partial_{-} F_{i h}(\hat{x})}{\partial x_{j h^{\prime}}} \mathbb{1}_{\Delta x_{j h^{\prime}}>0}\right] \Delta x_{j h^{\prime}}$. Thus we have $m_{i h+}^{\prime}\left(t_{i h}\right)$ and $m_{i h-}^{\prime}\left(t_{i h}\right)$ must be within interval $\left[-M_{i h}, M_{i h}\right]$ where

$$
M_{i h} \equiv \sum_{j, h^{\prime}} \max \left(\left|\frac{\partial_{+} F_{i h}(\hat{x})}{\partial x_{j h^{\prime}}}\right|,\left|\frac{\partial_{-} F_{i h}(\hat{x})}{\partial x_{j h^{\prime}}}\right|\right)\left|\Delta x_{j h^{\prime}}\right| .
$$

Thus $F_{i h}\left(x^{\prime}\right)-F_{i h}(x)=m_{i h}(1)-m_{i h}(0)$ must be also within interval $\left[-M_{i h}, M_{i h}\right]$. That is

$$
\begin{aligned}
\left|F_{i h}\left(x^{\prime}\right)-F_{i h}(x)\right| & \leq \sum_{j, h^{\prime}} \max \left(\left|\frac{\partial_{+} F_{i h}(\hat{x})}{\partial x_{j h^{\prime}}}\right|,\left|\frac{\partial_{-} F_{i h}(\hat{x})}{\partial x_{j h^{\prime}}}\right|\right)\left|\Delta x_{j h^{\prime}}\right| \\
& \leq \sum_{h^{\prime}}(\mathbf{A})_{h h^{\prime}} \max _{j}\left|\Delta x_{j h^{\prime}}\right| .
\end{aligned}
$$

Since the above expression holds for any $i, h$, we have $\max _{j}\left|F_{i h}\left(x^{\prime}\right)-F_{i h}(x)\right| \leq \sum_{h^{\prime}}(\mathbf{A})_{h h^{\prime}} \max _{j}\left|\Delta x_{j h^{\prime}}\right|$. Thus we establish a contraction mapping as in Theorem A.1, which gives us the existence and uniqueness of the solution in equation $x_{i h}=F_{i h}(x)$.

\section{B.1.4 Remark 4}

Consider first the equilibrium system (2) with constant elasticities, which can be written as follows:

$$
\lambda_{h} x_{i h}=\sum_{j \in \mathcal{N}} K_{i j h} \prod_{h^{\prime} \in \mathcal{H}} x_{j h^{\prime}}^{\alpha_{h h^{\prime}}}
$$

where $\lambda_{h}>0$ is endogenous. If $\rho(\mathbf{A}) \leq 1$, we have the same conclusion as in part (ii)b: the $\left\{x_{i h}\right\}$ of any solution is column-wise up-to-scale unique. The proof of this result is exactly the same as part (ii)b of Theorem 1.

For $\rho(\mathbf{A})<1$, particularly it is possible to subsume the endogenous scalars into the equilibrium outcomes through a change in variables, expressing equation (44) as in equation (1). To do so, define

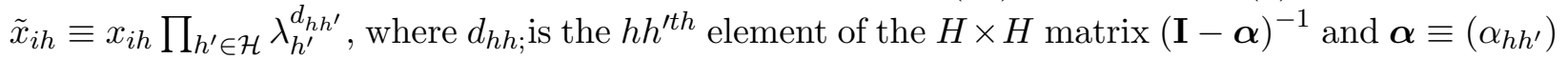
(i.e. $\boldsymbol{\alpha}$ is the matrix of elasticities without the absolute value taken) so the system becomes:

$$
\tilde{x}_{i h}=\sum_{j \in \mathcal{N}} K_{i j h} \prod_{h^{\prime} \in \mathcal{H}} \tilde{x}_{j h^{\prime}}^{\alpha_{h h^{\prime}}}
$$

Note that because $\rho(\mathbf{A})<1$, then so too is $\rho(\boldsymbol{\alpha})<1$, so that $(\mathbf{I}-\boldsymbol{\alpha})^{-1}$ exists. From Theorem 1 part (i), the $\left\{\tilde{x}_{i h}\right\}$ are unique and can be calculated using an iterative algorithm, which in turn implies that the $\left\{x_{i h}\right\}$ are column-wise up-to-scale unique. (Separating the $\left\{x_{i h}\right\}$ and $\left\{\lambda_{h}\right\}$ to determine the scale of $\left\{x_{i h}\right\}$ requires the imposition of further equilibrium conditions, e.g. aggregate labor market clearing conditions).

Consider now equilibrium system (2) with $H$ additional aggregate constraints $\sum_{i=1}^{N} x_{i h}=c_{h}$ 
for known constants $c_{h}>0$.

The second result concerns the general case with an endogenous scalar:

$$
\lambda_{h} x_{i h}=\sum_{j=1}^{N} f_{i j h}\left(x_{j 1}, \ldots, x_{j H}\right)
$$

with $H$ additional aggregate constraints $\sum_{i=1}^{N} x_{i h}=c_{h}$ for known constants $c_{h}>0$. Substituting in the aggregate constraints allows us to express the equilibrium system as:

$$
x_{i h}=\sum_{j=1}^{N}\left(\frac{f_{i j h}\left(x_{j 1}, \ldots, x_{j H}\right)}{\frac{1}{c_{h}} \sum_{i^{\prime}=1}^{N} \sum_{j^{\prime}=1}^{N} f_{i^{\prime} j^{\prime} h}\left(x_{j^{\prime} 1}, \ldots, x_{j^{\prime} H}\right)}\right),
$$

where the denominator is equal to the endogenous scalar, i.e. $\lambda_{h}=\frac{1}{c_{h}} \sum_{i^{\prime}=1}^{N} \sum_{j^{\prime}=1}^{N} f_{i^{\prime} j^{\prime} h}\left(x_{j^{\prime} 1}, \ldots, x_{j^{\prime} H}\right)$. We can define the new function:

$$
g_{i j, h}(x) \equiv \frac{f_{i j h}\left(x_{j 1}, \ldots, x_{j H}\right)}{\frac{1}{c_{h}} \sum_{i^{\prime}=1}^{N} \sum_{j^{\prime}=1}^{N} f_{i^{\prime} j^{\prime} h}\left(x_{j^{\prime} 1}, \ldots, x_{j^{\prime} H}\right)}
$$

so that the equilibrium system becomes:

$$
x_{i h}=\sum_{j=1}^{N} g_{i j h}(x) .
$$

We can then bound the elasticities, following Remark 1. Note:

$$
\frac{\partial \ln g_{i j, h}}{\partial \ln x_{m, l}}=\frac{\partial \ln f_{i j, h}}{\partial \ln x_{j, l}} \mathbf{1}_{m=j}-\sum_{o}\left(\frac{\partial \ln f_{o m, h}}{\partial \ln x_{m, l}}\right) \frac{f_{o m, h}\left(\left\{x_{m, l}\right\}\right)}{\sum_{o} \sum_{p} f_{o p, h}\left(\left\{x_{p, l}\right\}\right)}
$$

where $\mathbf{1}_{m=j}=\left\{\begin{array}{ll}1 & \text { if } m=j \\ 0 & \text { if } m \neq j\end{array}\right.$ is an indicator function. Thus,

$$
\left|\frac{\partial \ln g_{i j, h}}{\partial \ln x_{m, l}}\right| \leq\left|A_{h l}\right| \mathbf{1}_{m=j}+\left|A_{h l}\right| \frac{\sum_{o} f_{o m, h}\left(\left\{x_{m, l}\right\}\right)}{\sum_{o} \sum_{p} f_{o p, h}\left(\left\{x_{p, l}\right\}\right)} .
$$

Furthermore,

$$
\sum_{m}\left|\frac{\partial \ln g_{i j, h}}{\partial \ln x_{m, l}}\right| \leq \sum_{m}\left|A_{h l}\right| \mathbf{1}_{m=j}+\left|A_{h l}\right| \frac{\sum_{m} \sum_{o} f_{o m, h}\left(\left\{x_{m, l}\right\}\right)}{\sum_{o} \sum_{p} f_{o p, h}\left(\left\{x_{p, l}\right\}\right)}=2\left|A_{h l}\right| .
$$

Hence, from Remark 1, we have uniqueness as long as $\rho(\mathbf{A})<\frac{1}{2}$, as required.

\section{B.1.5 Remark 6}

Consider equation (3). We will directly prove that $\rho(\mathbf{A})=\rho\left(\mathbf{B} \boldsymbol{\Gamma}^{-1}\right) \geq 1$. Suppose for some $\bar{h} \geq 1$ that $\left\{x_{. h}\right\}_{h=1, \ldots, \bar{h}}$ are nominal variables. Then if we construct $\left\{\bar{x}_{. h}\right\}_{h \in \mathcal{H}}$ by scaling $\left\{x_{. h}\right\}_{h=1, \ldots, \bar{h}}$ up to t times and keeping all other entries unchanged, the constructed $\left\{\bar{x}_{. h}\right\}_{h \in \mathcal{H}}$ should still solve the equation. Therefore we can write 


$$
\boldsymbol{\Gamma} T=\mathbf{B} T
$$

where $T$ is a $H$-by-1 vector and

$$
T_{h}=\left\{\begin{array}{ll}
t & h \leq \bar{h} \\
0 & \text { other case }
\end{array} .\right.
$$

Notice that this further implies $\boldsymbol{\Gamma}^{-1} \mathbf{B}$ has eigenvalue of 1. Furthermore, because $\mathbf{B} \boldsymbol{\Gamma}^{-1}=\boldsymbol{\Gamma}\left(\boldsymbol{\Gamma}^{-1} \mathbf{B}\right) \boldsymbol{\Gamma}^{-1}$, $\mathbf{B} \boldsymbol{\Gamma}^{-1}$ also has eigenvalue of 1 . We define matrix $\mathbf{A}$ as the absolute value of $\mathbf{B} \boldsymbol{\Gamma}^{-1}$ (i.e. each entry of matrix $\mathbf{A}$ is the absolute value of the corresponding entry in matrix $\left.\mathbf{B} \boldsymbol{\Gamma}^{-1}\right)$. Therefore $\rho(\mathbf{A})$ must be weakly larger than 1 because $\rho(\mathbf{A})=\lim _{n \rightarrow \infty}\left\|\mathbf{A}^{n}\right\|^{\frac{1}{n}} \geq \lim _{n \rightarrow \infty}\left\|\left(\mathbf{B} \boldsymbol{\Gamma}^{-1}\right)^{n}\right\|^{\frac{1}{n}}=\rho\left(\mathbf{B} \boldsymbol{\Gamma}^{-\mathbf{1}}\right)$.

\section{B.1.6 Remark 7}

We prove a necessary and sufficient condition such that $\rho(\mathbf{A}) \leq 1$.

Lemma 1. Let $\mathbf{A}$ be a non-negative $n \times n$ matrix. The function $f(\lambda)$ is defined as the determinant of matrix $\lambda I-\mathbf{A}$ i.e. $f(\lambda)=|\lambda I-\mathbf{A}|$, and its $k$-th derivative is denoted by $f^{(k)}(\lambda)$. Then $\rho(\mathbf{A}) \leq s$ if and only if $f^{(k)}(s) \geq 0$ for all $k=0,1,2, \ldots, n-1$.

Proof. If part: Notice that $f^{(n)}(s)=n !>0$. Then $f^{(n-1)}(\lambda)$ strictly increases with $\lambda$. So $f^{(n-1)}(\lambda)>0$ for $\lambda \in[s, \infty)$. Using deduction we obtain $f(\lambda)$ is strictly increasing and $f(\lambda) \geq 0$ for any $\lambda \in[s, \infty]$. According to Perron-Frobenius theorem, $\rho(\mathbf{A})$ is $\mathbf{A}$ 's largest eigenvalue, so that $f(\rho(\mathbf{A}))=0$. Thus, by strict monotonicity it must be $\rho(\mathbf{A}) \leq s$.

Only If part: According to the Fundamental Theorem of Algebra (e.g. see Corollary 3.6.3 of Fine and Rosenberger (1997)), $f(\lambda)$ can be decomposed as $f(\lambda)=f_{1}(\lambda) f_{2}(\lambda)$ such that $f_{1}(\lambda)=$ $\prod_{i \in C}\left(\lambda-\lambda_{i}\right)\left(\lambda-\overline{\lambda_{i}}\right)$ and $f_{2}(\lambda)=\prod_{i \in R}\left(\lambda-\lambda_{i}\right)$ where $\overline{\lambda_{i}}$ is conjugate of $\lambda_{i}$ and $C$ and $R$ are set of indexes. For all $i \in C, \lambda_{i}$ is a complex number and for all $i \in R \lambda_{i}$ is a real number. Clearly, $\lambda_{i}$ and $\overline{\lambda_{i}}$ are eigenvalues of A.Notice that $f^{(k)}(\lambda)=\sum_{\left(k_{1}, k_{2}\right) \in D_{k}}, f_{1}^{\left(k_{1}\right)}(\lambda) f_{2}^{\left(k_{2}\right)}(\lambda)$ where $D_{k}=\left\{k_{1}, k_{2} \mid k_{1}+k_{2}=k, k_{1}, k_{2} \geq 0\right\}$. When $i \in R \lambda_{i} \leq \rho(\mathbf{A})$ (from Perron-Frobenius theorem), we have $f_{2}^{\left(k_{2}\right)}(s) \geq 0$. Additionally, $f_{1}^{\left(k_{1}\right)}(\lambda)=\prod_{i \in C}\left[\lambda^{2}-\left(\lambda_{i}+\overline{\lambda_{i}}\right) \lambda+\lambda_{i} \bar{\lambda}_{i}{ }^{\left(k_{2, i}\right)}\right.$ where $k_{2, i} \geq 0$ and $\sum_{i \in C} k_{2, i}=k_{2}$. Notice that

$$
\left[s^{2}-\left(\lambda_{i}+\overline{\lambda_{i}}\right) s+\lambda_{i} \bar{\lambda}_{i}\right]^{\left(k_{2, i}\right)}=\left\{\begin{array}{ll}
s^{2}-\left(\lambda_{i}+\overline{\lambda_{i}}\right) s+\lambda_{i} \overline{\lambda_{i}}>0 & k_{2, i}=0 \\
2\left(s-\operatorname{Re}\left(\lambda_{i}\right)\right) & k_{2, i}=1 \\
2>0 & k_{2, i}=2 \\
0 & k_{2, i}>3
\end{array},\right.
$$

where $\operatorname{Re}\left(\lambda_{i}\right)$ is real part of $\lambda_{i}$. As $\operatorname{Re}\left(\lambda_{i}\right)<\left\|\lambda_{i}\right\| \leq \rho(\mathbf{A}) \leq s$ (the second inequality is also from Perron-Frobenius theorem), so $\left[s^{2}-\left(\lambda_{i}+\overline{\lambda_{i}}\right) s+\lambda_{i} \bar{\lambda}_{i}{ }^{\left(k_{2, i}\right)} \geq 0\right.$. In all, $f^{(k)}(s) \geq 0 k=$ $0,1,2, \ldots, n-1$.

\section{B.2 Details of the Urban Spatial Model}

\section{B.2.1 Theorem 1, part (i): General spillovers}

Substituting equations (5) and (6) into the equilibrium conditions (7) yields the following two equilibrium conditions: 


$$
\begin{aligned}
& L_{i h}^{W}=\sum_{j \in \mathcal{N}} \frac{\left(\frac{\bar{u}_{j h} \bar{A}_{i h}}{\mu_{j i h}}\right)^{\theta_{h}}\left(f_{h}^{A}\left(\left\{L_{i, h^{\prime}}^{W}\right\}_{h^{\prime} \in \mathcal{H}}\right)\right)^{\theta_{h}}\left(f_{h}^{u}\left(\left\{L_{j, h^{\prime}}^{R}\right\}_{h^{\prime} \in \mathcal{H}}\right)\right)^{\theta_{h}}}{\sum_{(i, j) \in \mathcal{N}^{2}}\left(\frac{\bar{u}_{j h} \bar{A}_{i h}}{\mu_{j i h}}\right)^{\theta_{h}}\left(f_{h}^{A}\left(\left\{L_{i, h^{\prime}}^{W}\right\}_{h^{\prime} \in \mathcal{H}}\right)\right)^{\theta_{h}}\left(f_{h}^{u}\left(\left\{L_{j, h^{\prime}}^{R}\right\}_{h^{\prime} \in \mathcal{H}}\right)\right)^{\theta_{h}}} \bar{L}_{h} \\
& L_{i h}^{R}=\sum_{j} \frac{\left(\frac{\bar{u}_{i h} \bar{A}_{j h}}{\mu_{i j h}}\right)^{\theta_{h}}\left(f_{h}^{u}\left(\left\{L_{i, h^{\prime}}^{R}\right\}_{h^{\prime} \in \mathcal{H}}\right)\right)^{\theta_{h}}\left(f_{h}^{A}\left(\left\{L_{j, h^{\prime}}^{W}\right\}_{h^{\prime} \in \mathcal{H}}\right)\right)^{\theta_{h}}}{\sum_{(i, j) \in \mathcal{N}^{2}}\left(\frac{\bar{u}_{j h} \bar{A}_{i h}}{\mu_{j i h}}\right)^{\theta_{h}}\left(f_{h}^{A}\left(\left\{L_{i, h^{\prime}}^{W}\right\}_{h^{\prime} \in \mathcal{H}}\right)\right)^{\theta_{h}}\left(f_{h}^{u}\left(\left\{L_{j, h^{\prime}}^{R}\right\}_{h^{\prime} \in \mathcal{H}}\right)\right)^{\theta_{h}} \bar{L}_{h}}
\end{aligned}
$$

Denote the left side of equations (45) and (46) as $g_{i h}^{W}$ and $g_{i h}^{R}$, respectively. Since $\ln g_{i h}^{W}=$ $\ln \sum_{j \in \mathcal{N}} L_{i j h}-\ln \sum_{(i, j) \in \mathcal{N}^{2}} L_{i j h}$ where $L_{i j h}=\left(\frac{\bar{u}_{j h} \bar{A}_{i h}}{\mu_{j i h}}\right)^{\theta_{h}}\left(f_{h}^{A}\left(\left\{L_{i, h^{\prime}}^{W}\right\}_{h^{\prime} \in \mathcal{H}}\right)\right)^{\theta_{h}}\left(f_{h}^{u}\left(\left\{L_{j, h^{\prime}}^{R}\right\}_{h^{\prime} \in \mathcal{H}}\right)\right)^{\theta_{h}}$ we have:

$$
\begin{aligned}
\frac{\partial \ln g_{i h}^{W}}{\partial \ln L_{j h^{\prime}}^{W}} & =\frac{\partial \ln \sum_{j \in \mathcal{N}} L_{i j h}-\partial \ln \sum_{(m, j) \in \mathcal{N}^{2}} L_{i j h}}{\partial \ln L_{j h^{\prime}}^{F}} \\
& =\frac{L_{i j h}}{\sum_{j \in \mathcal{N}} L_{i j h}} \frac{\partial \ln L_{i j h}}{\partial \ln L_{j h^{\prime}}^{F}}-\sum_{m} \frac{L_{m j h}}{\sum_{(m, j) \in \mathcal{N}^{2}} L_{i j h}} \frac{\partial \ln L_{m j h}}{\partial \ln L_{j h^{\prime}}^{F}}
\end{aligned}
$$

Since $0 \leq \frac{\partial \ln L_{i j h}}{\partial \ln L_{s h^{\prime}}^{W}} \leq \theta_{h} \alpha_{h h^{\prime}}$, we have $\sum_{j}\left|\frac{\partial \ln g_{i h}^{W}}{\partial \ln L_{j h^{\prime}}^{W}}\right| \leq 2 \theta_{h} \alpha_{h h^{\prime}}$. Similarly, we have $\sum_{j}\left|\frac{\partial \ln g_{i h}^{W}}{\partial \ln L_{j h^{\prime}}^{R}}\right| \leq$ $2 \theta_{h} \beta_{h h^{\prime}}, \sum_{j}\left|\frac{\partial \ln g_{i h}^{R}}{\partial \ln L_{j h^{\prime}}^{W}}\right| \leq 2 \theta_{h} \alpha_{h h^{\prime}}$, and $\sum_{j}\left|\frac{\partial \ln g_{i h}^{R}}{\partial \ln L_{j h^{\prime}}^{R}}\right| \leq 2 \theta_{h} \beta_{h h^{\prime}}$. Therefore by applying Remark 1, part (i) of Theorem 1 yields the following sufficient condition for uniqueness:

$$
\rho\left(\begin{array}{cc}
2 \theta \boldsymbol{\alpha} & 2 \boldsymbol{\theta} \boldsymbol{\beta} \\
2 \boldsymbol{\theta} \boldsymbol{\alpha} & 2 \boldsymbol{\theta} \boldsymbol{\beta}
\end{array}\right)<1
$$

To see that this condition is equivalent with $\rho(\boldsymbol{\theta}(\boldsymbol{\alpha}+\boldsymbol{\beta}))<\frac{1}{2}$, simplify spectral radius $\rho\left(\begin{array}{cc}2 \boldsymbol{\theta} \boldsymbol{\alpha} & 2 \boldsymbol{\theta} \boldsymbol{\beta} \\ 2 \boldsymbol{\theta} \boldsymbol{\alpha} & 2 \boldsymbol{\theta} \boldsymbol{\beta}\end{array}\right)$ as $r$ and denote its associated eigenvector as $\left(\begin{array}{c}v_{\alpha} \\ v_{\beta}\end{array}\right)$ where both $v_{\alpha}$ and $v_{\beta}$ are $H$-by-1 vectors. Thus we have

$$
\begin{gathered}
\left(\begin{array}{cc}
2 \boldsymbol{\theta} \boldsymbol{\alpha} & 2 \boldsymbol{\theta} \boldsymbol{\beta} \\
2 \boldsymbol{\theta} \boldsymbol{\alpha} & 2 \boldsymbol{\theta} \boldsymbol{\beta}
\end{array}\right)\left(\begin{array}{c}
v_{\alpha} \\
v_{\beta}
\end{array}\right)=r\left(\begin{array}{c}
v_{\alpha} \\
v_{\beta}
\end{array}\right) \Rightarrow \\
\left(\begin{array}{c}
2 \boldsymbol{\theta} \boldsymbol{\alpha} v_{\alpha}+2 \boldsymbol{\theta} \boldsymbol{\beta} v_{\beta} \\
2 \boldsymbol{\theta} \boldsymbol{\alpha} v_{\alpha}+2 \boldsymbol{\theta} \boldsymbol{\beta} v_{\beta}
\end{array}\right)=r\left(\begin{array}{c}
v_{\alpha} \\
v_{\beta}
\end{array}\right),
\end{gathered}
$$

which implies $v_{\alpha}=v_{\beta}$. Thus, $\frac{r}{2}$ is the spectral radius of matrix $\boldsymbol{\theta}(\boldsymbol{\alpha}+\boldsymbol{\beta})$ with $v_{\alpha}$ being its corresponding eigenvector. 


\section{B.2.2 Theorem 1, part (ii): Constant elasticity spillovers}

We first combine the spillover equations $f_{h}^{A}\left(\left\{L_{j, h^{\prime}}^{W}\right\}_{h^{\prime} \in \mathcal{H}}\right)=\prod_{h^{\prime} \in \mathcal{H}}\left(L_{j, h^{\prime}}^{W}\right)^{\alpha_{h h^{\prime}}}$ and $f_{h}^{u}\left(\left\{L_{i, h^{\prime}}^{R}\right\}_{h^{\prime} \in \mathcal{H}}\right)=$ $\prod_{h^{\prime} \in \mathcal{H}}\left(L_{i, h^{\prime}}^{R}\right)^{\beta_{h h^{\prime}}}$ with equation (5) into the equilibrium system defined by equation (7), we get:

$$
\begin{aligned}
& \frac{L_{i h}^{W}}{\left(\prod_{h^{\prime} \in \mathcal{H}}\left(L_{i, h^{\prime}}^{W}\right)^{\alpha_{h h^{\prime}}}\right)^{\theta_{h}}}=\lambda_{h} \sum_{j \in \mathcal{N}}\left(\frac{\bar{u}_{j h} \bar{A}_{i h}}{\mu_{j i h}}\right)^{\theta_{h}}\left(\prod_{h^{\prime} \in \mathcal{H}}\left(L_{j, h^{\prime}}^{R}\right)^{\beta_{h h^{\prime}}}\right)^{\theta_{h}} \\
& \frac{L_{i h}^{R}}{\left(\prod_{h^{\prime} \in \mathcal{H}}\left(L_{i, h^{\prime}}^{R}\right)^{\beta_{h h^{\prime}}}\right)^{\theta_{h}}}=\lambda_{h} \sum_{j}\left(\frac{\bar{u}_{i h} \bar{A}_{j h}}{\mu_{i j h}}\right)^{\theta_{h}}\left(\prod_{h^{\prime} \in \mathcal{H}}\left(L_{j, h^{\prime}}^{W}\right)^{\alpha_{h h^{\prime}}}\right)^{\theta_{h}}
\end{aligned}
$$

We then pursue the change in variables described in Remark 5. Define:

$$
x_{i h}^{W} \equiv\left(\frac{L_{i h}^{W}}{\prod_{h^{\prime} \in \mathcal{H}}\left(L_{i, h^{\prime}}^{W}\right)^{\alpha_{h h^{\prime}}}}\right)
$$

so that:

$$
\ln x_{i h}^{W}=\ln L_{i h}^{W}-\theta_{h} \sum_{h^{\prime} \in \mathcal{H}} \alpha_{h h^{\prime}} \ln L_{i h^{\prime}}^{W}
$$

or, in matrix notation:

$$
\begin{aligned}
\ln \boldsymbol{x}_{i}^{W} & =(\mathbf{I}-\boldsymbol{\theta} \boldsymbol{\alpha}) \ln \boldsymbol{L}_{i}^{W} \Longleftrightarrow \\
\ln \boldsymbol{L}_{i}^{W} & =(\mathbf{I}-\boldsymbol{\theta} \boldsymbol{\alpha})^{-1} \ln \boldsymbol{x}_{i}^{W}
\end{aligned}
$$

or, in non-vector notation, we have:

$$
L_{i h}^{W}=\prod_{h^{\prime} \in \mathcal{H}}\left(x_{i h^{\prime}}^{W}\right)^{a_{h h^{\prime}}^{-1}},
$$

where $\left[a_{h h^{\prime}}^{-1}\right] \equiv(\mathbf{I}-\boldsymbol{\theta} \boldsymbol{\alpha})^{-1}$.

Similarly, define:

$$
x_{i h}^{R} \equiv\left(\frac{L_{i h}^{R}}{\left(\prod_{h^{\prime} \in \mathcal{H}}\left(L_{i, h^{\prime}}^{R}\right)^{\beta_{h h^{\prime}}}\right)^{\theta_{h}}}\right)
$$

so that:

$$
\begin{aligned}
\ln \boldsymbol{x}_{i}^{R} & =(\mathbf{I}-\boldsymbol{\theta} \boldsymbol{\beta}) \ln \boldsymbol{L}_{i}^{R} \Longleftrightarrow \\
\ln \boldsymbol{L}_{i}^{R} & =(\mathbf{I}-\boldsymbol{\theta} \boldsymbol{\beta})^{-1} \ln \boldsymbol{x}_{i}^{R}
\end{aligned}
$$

or in non-vector notation:

$$
L_{i h}^{R}=\prod_{h^{\prime} \in \mathcal{H}}\left(x_{i h^{\prime}}^{R}\right)^{b_{h h^{\prime}}^{-1}}
$$


where $\left[b_{h h^{\prime}}^{-1}\right] \equiv(\mathbf{I}-\boldsymbol{\theta} \boldsymbol{\beta})^{-1}$. Then we can write:

$$
\begin{aligned}
\left(\frac{L_{i h}^{W}}{\left(\prod_{h^{\prime} \in \mathcal{H}}\left(L_{i, h^{\prime}}^{W}\right)^{\alpha_{h h^{\prime}}}\right)^{\theta_{h}}}\right) & =\lambda_{h} \sum_{j \in \mathcal{N}}\left(\frac{\bar{u}_{j h} \bar{A}_{i h}}{\mu_{j i h}}\right)^{\theta_{h}}\left(\prod_{h^{\prime} \in \mathcal{H}}\left(L_{j, h^{\prime}}^{R}\right)^{\beta_{h h^{\prime}}}\right)^{\theta_{h}} \Longleftrightarrow \\
x_{i h}^{W} & =\lambda_{h} \sum_{j \in \mathcal{N}}\left(\frac{\bar{u}_{j h} \bar{A}_{i h}}{\mu_{j i h}}\right)^{\theta_{h}}\left(\prod_{h^{\prime} \in \mathcal{H}}\left(\prod_{h^{\prime \prime} \in \mathcal{H}}\left(x_{j, h^{\prime \prime}}^{R}\right)^{b_{h^{\prime}, h^{\prime \prime}}}\right)^{\beta_{h h^{\prime}}}\right)^{\theta_{h}} \Longleftrightarrow \\
x_{i h}^{W} & =\lambda_{h} \sum_{j \in \mathcal{N}}\left(\frac{\bar{u}_{j h} \bar{A}_{i h}}{\mu_{j i h}}\right)^{\theta_{h}} \prod_{h^{\prime} \in \mathcal{H}}\left(x_{j, h^{\prime}}^{R}\right)^{\gamma_{h, h^{\prime}}^{R}}
\end{aligned}
$$

where $\left[\gamma_{h, h^{\prime}}^{R}\right] \equiv \boldsymbol{\theta} \boldsymbol{\beta}(\mathbf{I}-\boldsymbol{\theta} \boldsymbol{\beta})^{-1}$.

Similarly:

$$
\begin{aligned}
\left(\frac{L_{i h}^{R}}{\left(\prod_{h^{\prime} \in \mathcal{H}}\left(L_{i, h^{\prime}}^{R}\right)^{\beta_{h h^{\prime}}}\right)^{\theta_{h}}}\right) & =\lambda_{h} \sum_{j}\left(\frac{\bar{u}_{i h} \bar{A}_{j h}}{\mu_{i j h}}\right)^{\theta_{h}}\left(\prod_{h^{\prime} \in \mathcal{H}}\left(L_{j, h^{\prime}}^{W}\right)^{\alpha_{h h^{\prime}}}\right)^{\theta_{h}} \Longleftrightarrow \\
x_{i h}^{R} & =\lambda_{h} \sum_{j}\left(\frac{\bar{u}_{i h} \bar{A}_{j h}}{\mu_{i j h}}\right)^{\theta_{h}}\left(\prod_{h^{\prime} \in \mathcal{H}}\left(\prod_{h^{\prime \prime} \in \mathcal{H}}\left(x_{j h^{\prime \prime}}^{W}\right)^{a_{h^{\prime} h^{\prime \prime}}}\right)^{\alpha_{h h^{\prime}}}\right)^{\theta_{h}} \Longleftrightarrow \\
x_{i h}^{R} & =\lambda_{h} \sum_{j}\left(\frac{\bar{u}_{i h} \bar{A}_{j h}}{\mu_{i j h}}\right)^{\theta_{h}} \prod_{h^{\prime} \in \mathcal{H}}\left(x_{j, h^{\prime}}^{W}\right)^{\gamma_{h, h^{\prime}}^{F} \theta_{h}}
\end{aligned}
$$

where $\left[\gamma_{h, h^{\prime}}^{W}\right] \equiv \boldsymbol{\theta} \boldsymbol{\alpha}(\mathbf{I}-\boldsymbol{\theta} \boldsymbol{\alpha})^{-1}$.

Finally, we apply the change of variables described in Remark 2 to subsume the endogenous scalars $\left\{\lambda_{h}\right\}$ in the equilibrium system. Define:

$$
\begin{aligned}
y_{i h}^{W} & \equiv\left(\prod_{h^{\prime}} \lambda_{h^{\prime}}^{c_{h^{\prime} h}^{W}}\right)^{-1} x_{i h}^{W} \\
y_{i h}^{R} & \equiv\left(\prod_{h^{\prime}} \lambda_{h^{\prime}}^{c_{h^{\prime} h}^{R}}\right)^{-1} x_{i h}^{R}
\end{aligned}
$$

so that we have:

$$
\begin{aligned}
& x_{i h}^{W}=\lambda_{h} \sum_{j \in \mathcal{N}}\left(\frac{\bar{u}_{j h} \bar{A}_{i h}}{\mu_{j i h}}\right)^{\theta_{h}} \prod_{h^{\prime} \in \mathcal{H}}\left(x_{j, h^{\prime}}^{R}\right)^{\theta_{h} \gamma_{h, h^{\prime}}^{R}} \Longleftrightarrow \\
& y_{i h}^{W}\left(\prod_{h^{\prime}} \lambda_{h^{\prime}}^{c_{h^{\prime} h}^{W}}\right)=\lambda_{h} \sum_{j \in \mathcal{N}}\left(\frac{\bar{u}_{j h} \bar{A}_{i h}}{\mu_{j i h}}\right)^{\theta_{h}} \prod_{h^{\prime} \in \mathcal{H}}\left(\prod_{h^{\prime \prime}} \lambda_{h^{\prime \prime}}^{c_{h^{\prime \prime}}^{R} h^{\prime}} y_{j, h^{\prime}}^{R}\right)^{\theta_{h} \gamma_{h, h^{\prime}}^{R}} \Longleftrightarrow \\
& y_{i h}^{W}=\sum_{j \in \mathcal{N}}\left(\frac{\bar{u}_{j h} \bar{A}_{i h}}{\mu_{j i h}}\right)^{\theta_{h}} \prod_{h^{\prime} \in \mathcal{H}}\left(y_{j, h^{\prime}}^{R}\right)^{\theta_{h} \gamma_{h, h^{\prime}}^{R}}
\end{aligned}
$$


and:

$$
\begin{aligned}
& x_{i h}^{R}=\lambda_{h} \sum_{j}\left(\frac{\bar{u}_{i h} \bar{A}_{j h}}{\mu_{i j h}}\right)^{\theta_{h}} \prod_{h^{\prime} \in \mathcal{H}}\left(x_{j, h^{\prime}}^{W}\right)^{\gamma_{h, h^{\prime}}^{W} \theta_{h}} \Longleftrightarrow \\
& y_{i h}^{R}\left(\prod_{h^{\prime}} \lambda_{h^{\prime}}^{c_{h^{\prime}}^{R}}\right)=\lambda_{h} \sum_{j}\left(\frac{\bar{u}_{i h} \bar{A}_{j h}}{\mu_{i j h}}\right)^{\theta_{h}} \prod_{h^{\prime} \in \mathcal{H}}\left(\prod_{h^{\prime \prime}} \lambda_{h^{\prime \prime}}^{c_{h^{\prime \prime} h^{\prime}}^{W}} y_{j, h^{\prime}}^{W}\right)^{\gamma_{h, h^{\prime}}^{W} \theta_{h}} \Longleftrightarrow \\
& y_{i h}^{R}=\sum_{j}\left(\frac{\bar{u}_{i h} \bar{A}_{j h}}{\mu_{i j h}}\right)^{\theta_{h}} \prod_{h^{\prime} \in \mathcal{H}}\left(y_{j, h^{\prime}}^{W}\right)^{\gamma_{h, h^{\prime}}^{W} \theta_{h}}
\end{aligned}
$$

We then need to choose $\left\{c_{h}^{W}, c_{h}^{R}\right\}$ such that:

$$
\begin{gathered}
\mathbf{I}-C^{R}+\left[\theta_{h} \gamma_{h, h^{\prime}}^{R}\right] C^{R}=0 \Longleftrightarrow \\
\left(\mathbf{I}-\left[\theta_{h} \gamma_{h, h^{\prime}}^{R}\right]\right)^{-1}=\mathbf{C}^{R}
\end{gathered}
$$

and similarly:

$$
\left(\mathbf{I}-\left[\theta_{h} \gamma_{h, h^{\prime}}^{W}\right]\right)^{-1}=\mathbf{C}^{W}
$$

Finally, defining $\tilde{L}_{i h}^{W} \equiv y_{i h}^{W}$ and $\tilde{L}_{i h}^{R} \equiv y_{i h}^{R}$, we recover the equilibrium system defined in equation (8), as required.

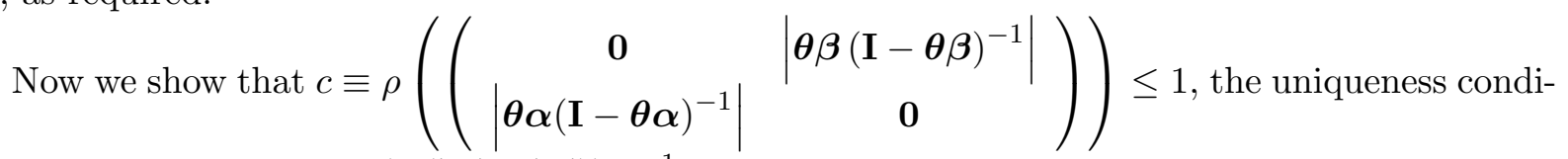

tion here, is weaker than $\rho(\boldsymbol{\theta}(|\boldsymbol{\alpha}|+|\boldsymbol{\beta}|))<\frac{1}{2}$, the condition required in the case of general spillovers given above. Suppose that for $v_{\alpha}$ and $v_{\beta} H$-by-1 vectors,

$$
\begin{aligned}
& \left(\begin{array}{c|c}
\mathbf{0} & \left|\boldsymbol{\theta} \boldsymbol{\beta}(\mathbf{I}-\boldsymbol{\theta} \boldsymbol{\beta})^{-1}\right| \\
\left|\boldsymbol{\theta} \boldsymbol{\alpha}(\mathbf{I}-\boldsymbol{\theta} \boldsymbol{\alpha})^{-1}\right| & \mathbf{0}
\end{array}\right)\left(\begin{array}{c}
v_{\alpha} \\
v_{\beta}
\end{array}\right)=c\left(\begin{array}{c}
v_{\alpha} \\
v_{\beta}
\end{array}\right) \Rightarrow \\
& \left(\begin{array}{c|c}
\mid \boldsymbol{\theta} \boldsymbol{\beta}(\mathbf{I}-\boldsymbol{\theta} \boldsymbol{\beta})^{-1} & v_{\beta} \\
\boldsymbol{\theta} \boldsymbol{\alpha}(\mathbf{I}-\boldsymbol{\theta} \boldsymbol{\alpha})^{-1} & v_{\alpha}
\end{array}\right)=c\left(\begin{array}{c}
v_{\alpha} \\
v_{\beta}
\end{array}\right) \Rightarrow \\
& \left|\boldsymbol{\theta} \boldsymbol{\alpha}(\mathbf{I}-\boldsymbol{\theta} \boldsymbol{\alpha})^{-1}\right|\left|\boldsymbol{\theta} \boldsymbol{\beta}(\mathbf{I}-\boldsymbol{\theta} \boldsymbol{\beta})^{-1}\right| v_{\beta}=c^{2} v_{\beta} .
\end{aligned}
$$

Thus it is equivalent to show that $\rho\left(\left|\boldsymbol{\theta} \boldsymbol{\alpha}(\mathbf{I}-\boldsymbol{\theta} \boldsymbol{\alpha})^{-1}\right|\left|\boldsymbol{\theta} \boldsymbol{\beta}(\mathbf{I}-\boldsymbol{\theta} \boldsymbol{\beta})^{-1}\right|\right)=c^{2} \leq 1$. Define $H$-by$H$ matrix $\boldsymbol{\delta}$ where $(\boldsymbol{\delta})_{h h^{\prime}}=\max \left((\boldsymbol{\theta}|\boldsymbol{\alpha}|)_{h h^{\prime}},(\boldsymbol{\theta}|\boldsymbol{\beta}|)_{h h^{\prime}}\right)$. Clearly, $\left|\boldsymbol{\theta} \boldsymbol{\beta}(\mathbf{I}-\boldsymbol{\theta} \boldsymbol{\beta})^{-1}\right| \leq \sum_{n=1}^{\infty}(\boldsymbol{\theta}|\boldsymbol{\beta}|)^{n} \leq$ $\sum_{n=1}^{\infty} \boldsymbol{\delta}^{n}$ where the inequality is elementwise; similarly, $\left|\boldsymbol{\theta} \boldsymbol{\alpha}(\mathbf{I}-\boldsymbol{\theta} \boldsymbol{\alpha})^{-1}\right| \leq \sum_{n=1}^{\infty} \boldsymbol{\delta}^{n}$. Thus,

$$
\rho\left(\left|\boldsymbol{\theta} \boldsymbol{\alpha}(\mathbf{I}-\boldsymbol{\theta} \boldsymbol{\alpha})^{-1}\right|\left|\boldsymbol{\theta} \boldsymbol{\beta}(\mathbf{I}-\boldsymbol{\theta} \boldsymbol{\beta})^{-1}\right|\right) \leq \rho\left(\sum_{n=1}^{\infty} \boldsymbol{\delta}^{n} \sum_{n=1}^{\infty} \boldsymbol{\delta}^{n}\right)=\rho\left(\sum_{n=1}^{\infty} \boldsymbol{\delta}^{n}\right)^{2} .
$$

Here, $\rho\left(\sum_{n=1}^{\infty} \boldsymbol{\delta}^{n}\right)=\frac{\rho(\boldsymbol{\delta})}{1-\rho(\boldsymbol{\delta})}$. Furthermore, $\rho(\boldsymbol{\delta}) \leq \rho(\boldsymbol{\theta}(|\boldsymbol{\alpha}|+|\boldsymbol{\beta}|))<\frac{1}{2}$. Thus, $\rho\left(\sum_{n=1}^{\infty} \boldsymbol{\delta}^{n}\right)<1$, which is as desired. 
Figure 1: Examples of multiplicity in an urban spatial framework
(a) $\mu=2, \alpha=\beta=0.5$
(b) $\mu=2, \alpha=\beta=0.55$
(c) $\mu=2, \alpha=\beta=0.6$

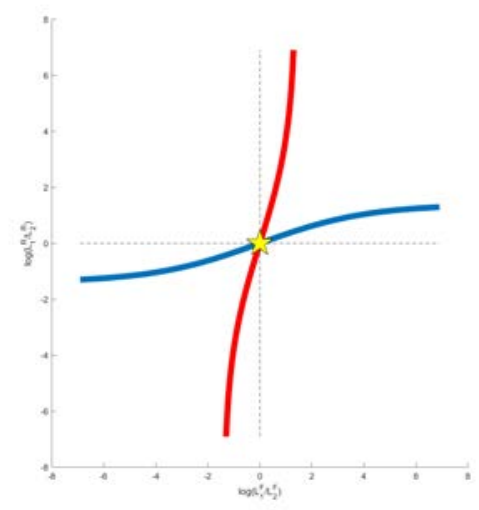

(d) $\mu=6, \alpha=\beta=0.5$

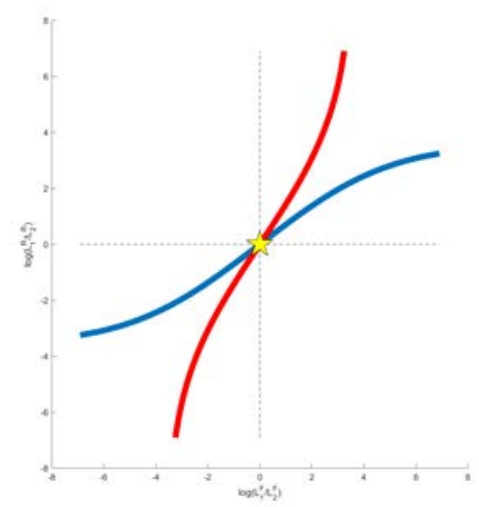

(g) $\mu=11, \alpha=\beta=0.5$

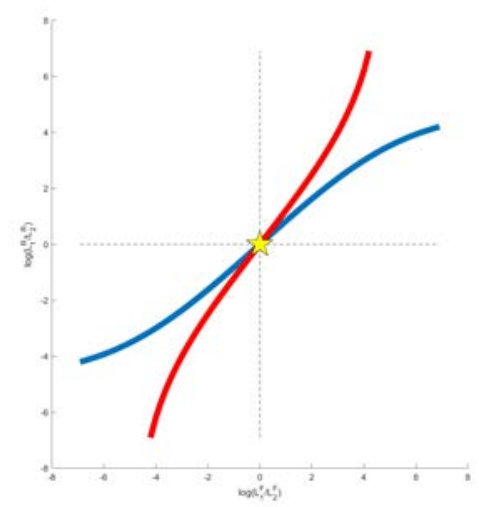

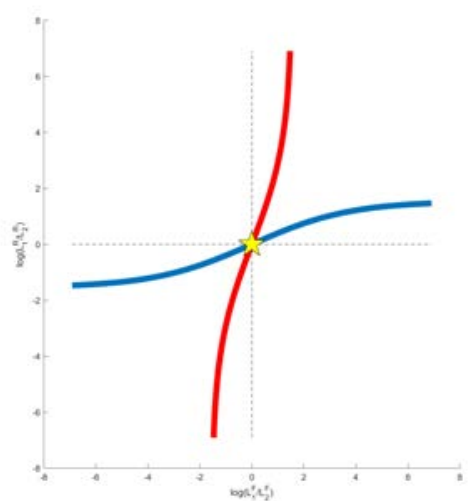

(e) $\mu=6, \alpha=\beta=0.55$

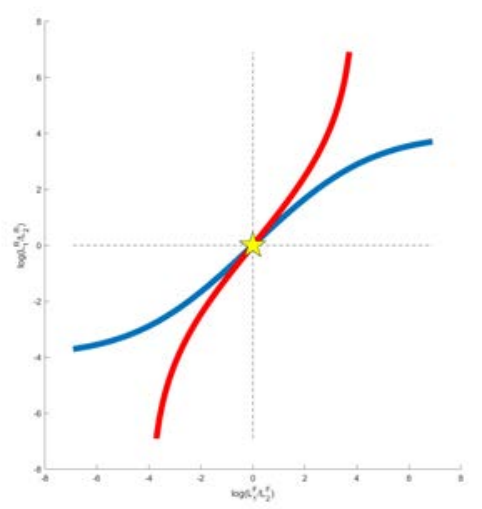

(h) $\mu=11, \alpha=\beta=0.55$

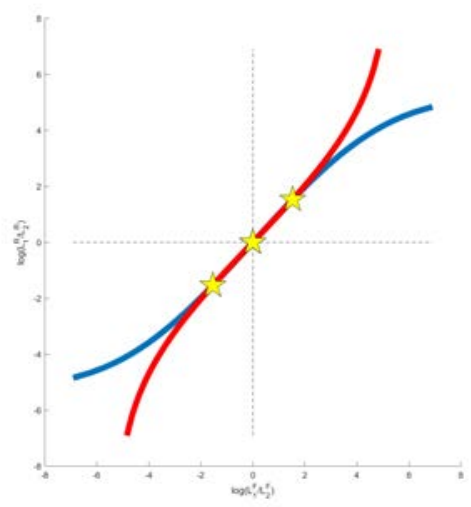

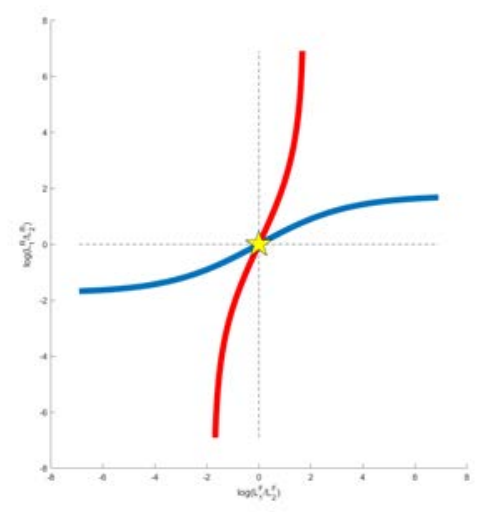

(f) $\mu=6, \alpha=\beta=0.6$

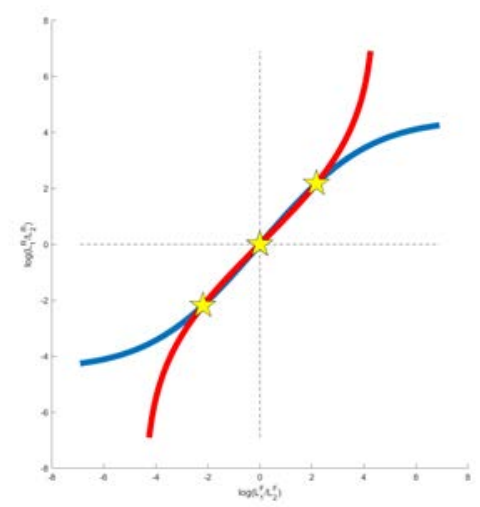

(i) $\mu=11, \alpha=\beta=0.6$

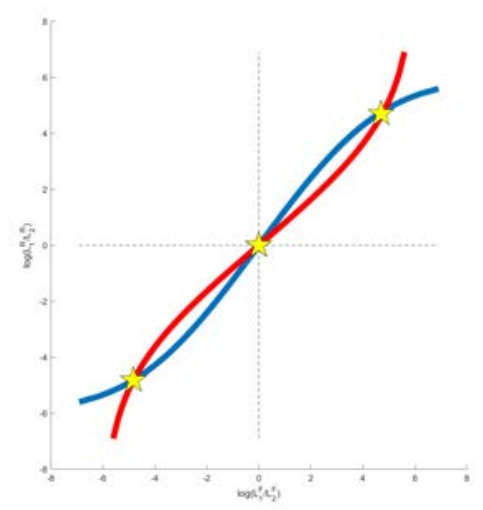

Notes: This figure depicts the set equilibria for an urban economy with two identical locations and a single type of agent for different combinations of productivity and amenity spillovers ( $\alpha$ and $\beta$, respectively) and commuting costs $(\mu)$. The $\mathrm{x}$-axis is the (log) ratio of the workers in location 1 relative to location 2 ; the y-axis is the (log) ratio of residents in location 1 relative to location 2. Stars indicate an equilibrium. 